2014

\title{
CHARACTERIZATION OF THE ATMOSPHERIC EFFECTS ON THE TRANSMISSION OF THERMAL RADIATION
}

Mohamed E. Hanafy

Michigan Technological University

Follow this and additional works at: https://digitalcommons.mtu.edu/etds

Part of the Atmospheric Sciences Commons, Electrical and Computer Engineering Commons, and the Optics Commons

Copyright 2014 Mohamed E. Hanafy

\section{Recommended Citation}

Hanafy, Mohamed E., "CHARACTERIZATION OF THE ATMOSPHERIC EFFECTS ON THE TRANSMISSION OF THERMAL RADIATION", Dissertation, Michigan Technological University, 2014.

https://doi.org/10.37099/mtu.dc.etds/854

Follow this and additional works at: https://digitalcommons.mtu.edu/etds

Part of the Atmospheric Sciences Commons, Electrical and Computer Engineering Commons, and the Optics Commons 


\title{
CHARACTERIZATION OF THE ATMOSPHERIC EFFECTS ON THE TRANSMISSION OF THERMAL RADIATION
}

\author{
By \\ Mohamed E. Hanafy

\begin{abstract}
A DISSERTATION
Submitted in partial fulfillment of the requirements for the degree of DOCTOR OF PHILOSOPHY

In Electrical Engineering
\end{abstract}

MICHIGAN TECHNOLOGICAL UNIVERSITY

2014

(C) 2014 Mohamed E. Hanafy 

This dissertation has been approved in partial fulfillment of the requirements for the Degree of DOCTOR OF PHILOSOPHY in Electrical Engineering.

Department of Electrical and Computer Engineering

Dissertation Co-advisor: $\quad$ Professor/Michael C. Roggemann

Dissertation Co-advisor: $\quad$ Assistant Professor/ Durdu O. Guney

Committee Member: $\quad$ Associate Professor/Christopher T. Middlebrook

Committee Member: $\quad$ Professor/Raymond A. Shaw

Department Chair: $\quad$ Professor/Dan Fuhrmann 

To the country where human civilization was first risen, Egypt. 



\section{Table of Contents}

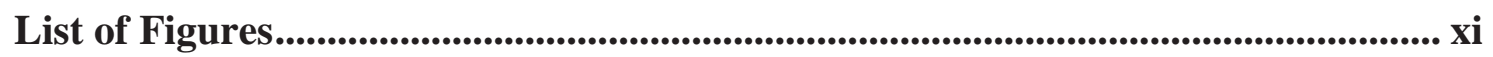

List of Tables ............................................................................................................................

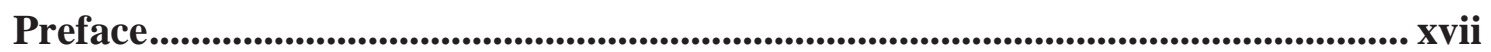

Acknowledgments ......................................................................................................................... xix

Abstract........................................................................................................................................... xxi

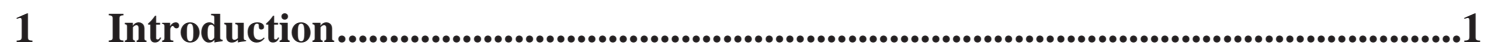

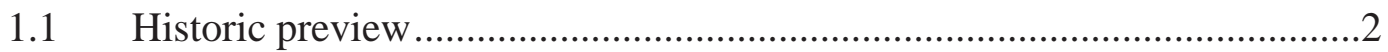

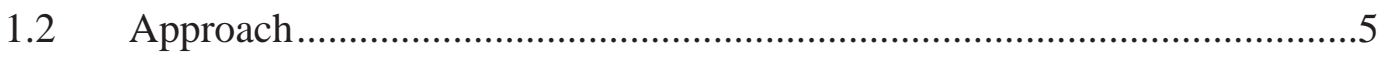

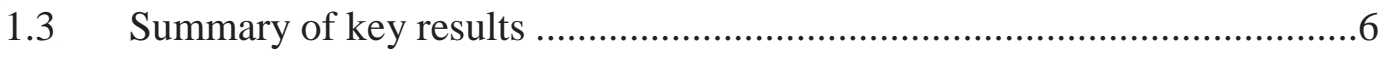

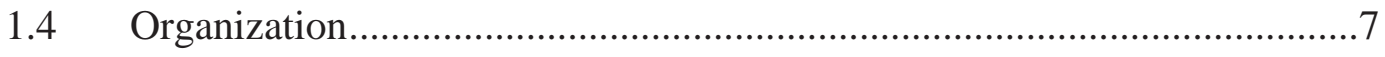

2 Detailed Effects of Scattering and Absorption by Haze and Aerosols in

The Atmosphere on The Average Point Spread Function of an

Imaging System ............................................................................................................................10

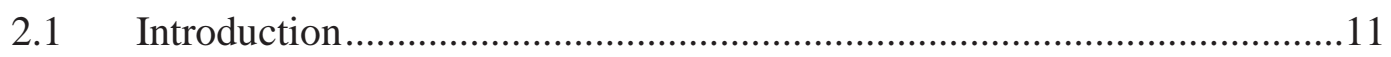

2.2 Optical properties of a single spherical particle.........................................13

2.2.1 EM efficiency factors...................................................................13

2.2.2 Single Scattering Albedo ……………………………..................18

2.2.3 Scattering Phase Function..................................................................19

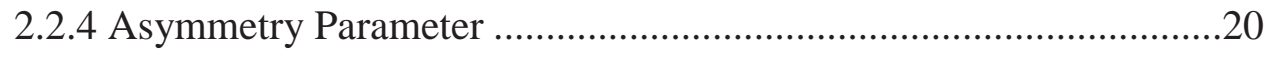

2.3 Atmospheric aerosol models ............................................................23 
2.3.1 Particle size distribution function ............................................23

2.3.2 Atmospheric optical properties ...................................................24

2.3.3 Haze and fog atmospheric aerosol models..................................26

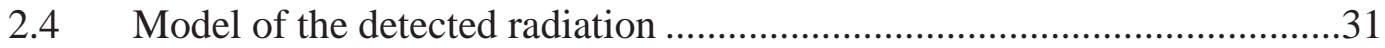

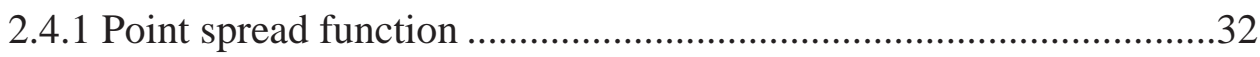

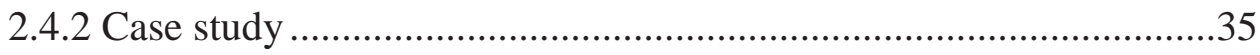

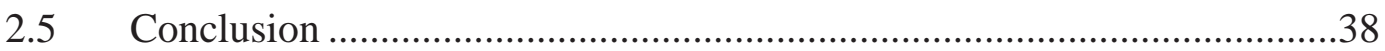

3 Estimating The Image Spectrum Signal-to-Noise Ratio for Imaging Through Scattering Media ...........................................................................................40

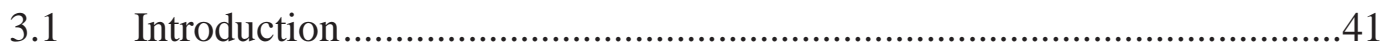

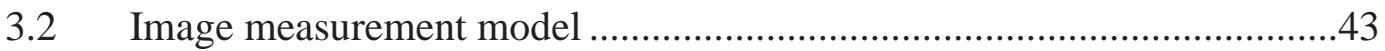

3.3 Effective point spread function .................................................... 48

3.4 Image spectrum signal-to-noise ratio calculations................................52

3.4.1 Calculation of the numbers of direct and scattered photons .............54

3.4.2 Calculation of the image spectrum SNR...................................56

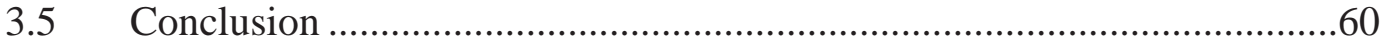

4 Reconstruction of Images Degraded by Aerosol Scattering and

Measurement Noise...................................................................................62

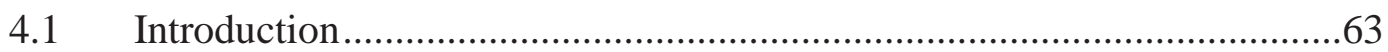

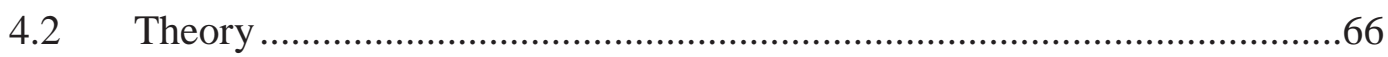

4.2.1 Effective point spread function ..............................................6 67

4.2.2 Modulation transfer function .................................................. 71

4.3 Image simulation and reconstruction ............................................... 73

viii 
4.3.1 Image spectrum signal-to-noise ratio.

4.3.2 Image reconstruction.

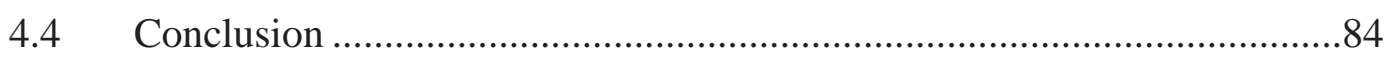

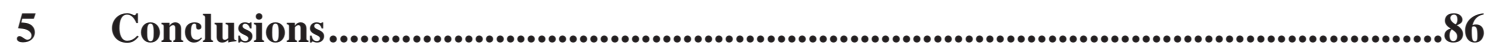

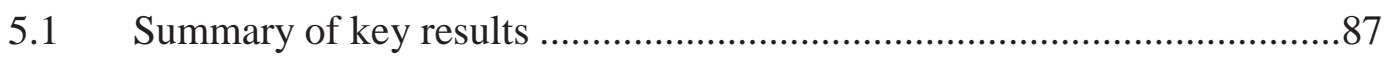

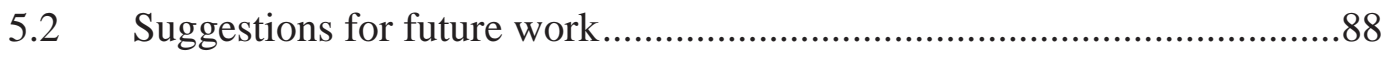

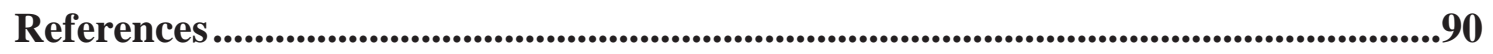

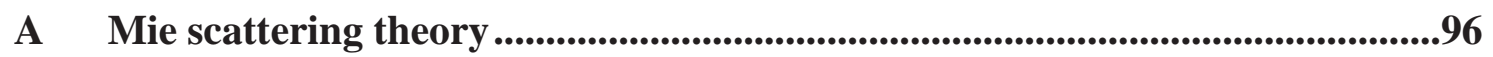

B Spectral Refractive Indices of Particles Constituting Haze and Fog Atmospheric Aerosol Models .....................................................................103

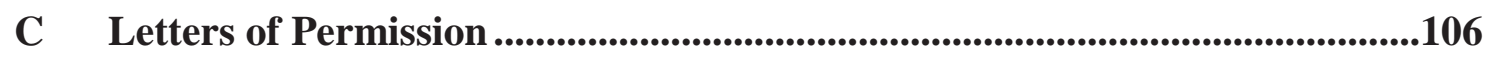

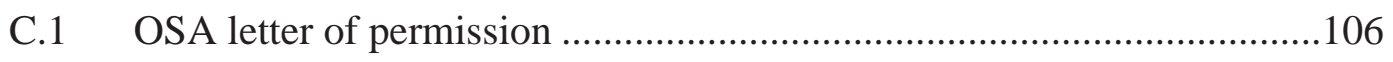

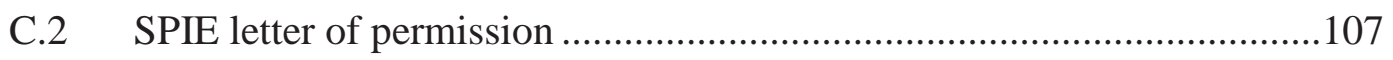




\section{List of Figures}

2.1 Extinction efficiency factor, $Q_{e x t}$ Scattering efficiency factor, $Q_{s c a}$, and Absorption efficiency factor, $Q_{a b s}$, versus size parameter, $x$, for a spherical particle with $m^{\prime}=1.33$, and various $m^{\prime \prime}$ values, at visible wavelength.

2.2 Extinction efficiency factor, $Q_{e x}$, Scattering efficiency factor, $Q_{s c a}$, and Absorption efficiency factor, $Q_{a b s}$, versus size parameter, $x$, for a spherical particle with $m^{\prime \prime}=0.1$, and various $m^{\prime}$ values, at visible wavelength.

2.3 Single scattering albedo, $\omega_{o}$, versus size parameter, $x$, at visible wavelength, for (a) different values of the imaginary part of refractive index $m^{\prime \prime}$, and (b) different values of the real part of refractive index $m^{\prime}$.

2.4 Single-scattering phase function, $P(\theta)$, for differently sized particles with refractive index $m=1.33+\mathrm{i} 10^{-8}$, at visible wavelength.

2.5 Asymmetry parameter, $g$, versus particle size parameter, $x$, at visible wavelength, for (a) different values of the imaginary part of refractive index $m^{\prime \prime}$, and (b) different values of the real part of refractive index $m^{\prime}$.

2.6 Bi-modal lognormal size distribution function for haze aerosol models in accordance with data listed in table (2.1)

2.7 Modified Gamma size distribution function for fog aerosol models in accordance with data listed in table (2.2).

2.8 Optical properties of haze aerosol models in accordance with parameters listed in table (2.1) 
2.9 Optical properties of fog aerosol models in accordance with parameters listed in table (2.2). 30

2.10 Phase function for tropospheric haze, moderate radiation fog, and heavy advection fog aerosol models

2.11 Angular distribution of the detected PSFs for fog aerosol models in accordance with the data listed in table (2.3) 36

3.1 Geometry of the photon detection method.

3.2 Solar spectral irradiance $E(\lambda)$ at the visible-NIR bands, and thermal spectral exitance $M(\lambda)$ at NIR-MIR bands for an extended target.

3.3 Optical properties of the haze and fog atmospheric aerosol models.

3.4 $P S F_{\text {dir }}(0, \lambda)$ and $P S F_{\text {sca }}(0, \lambda)$ for atmospheric aerosol models along different values of range $R$

3.5 Average number of direct and scattered photons, $K_{d i r}$ and $K_{s c a}$, for atmospheric aerosol models along different values of range $R$. 55

3.6 Radially-averaged image spectrum signal-to-noise ratio, $S N R_{r a d}$, for haze and fog aerosol models at the visible, NIR and MIR spectral wavelength bands.

4.1 Phase function of the atmospheric fog aerosol models at visible wavelength band.

4.2 Radial distribution of the normalized total effective PSF for the fog aerosol models at different ranges, $R$, between the target and imaging system values at the visible wavelength. 
4.3 Sampling parameters for the total modulation transfer function, $M T F_{t}$, of the fog aerosol models.

4.4 Modulation transfer function, MTF, at visible wavelength, for a diffraction-limited imaging system $M T F_{\text {diff, with }} D=0.02[\mathrm{~m}]$ and $f=0.1$ $[m]$, and for atmospheric fog aerosol models, $M T F_{t}$, at different ranges, $R$, between target and imaging system.

4.5 Images degraded by scattering due to atmospheric fog aerosol models, and corrupted with Poisson noise at different ranges, $R$, between target and imaging system.

4.6 Reconstruction of degraded images of figure (4.5) using Wiener filter.

4.7 Reconstruction of degraded images of figure (4.5) using constrained least squares (CLS) filter.

4.8 Reconstruction of degraded images of figure (4.6) using constrained least squares (CLS) filter.

A.1 Geometry for scattering of an electromagnetic wave by a spherical particle

A.2 Angle-dependent functions $\pi_{n}(\cos \theta)$ and $\tau_{n}(\cos \theta)$ used in calculating the scattering amplitude functions $S_{1}(\theta)$ and $S_{2}(\theta)$.

B.1 Maritime haze aerosols, $\mathrm{RH}=90 \%$, real and imaginary parts, $m^{\prime}$ and $m^{\prime \prime}$, of the refractive index, $m=m^{\prime}+i m^{\prime \prime}$.

B.2 Urban haze aerosols, $\mathrm{RH}=80 \%$, real and imaginary parts, $m^{\prime}$ and $m^{\prime \prime}$, of the refractive index, $m=m^{\prime}+i m^{\prime \prime}$. 
B.3 Rural haze aerosols, $\mathrm{RH}=70 \%$, real and imaginary parts, $m^{\prime}$ and $m^{\prime \prime}$, of the refractive index, $m=m^{\prime}+i m^{\prime \prime}$

B.4 Tropospheric haze aerosols, $\mathrm{RH}=50 \%$, real and imaginary parts, $m^{\prime}$ and $m^{\prime \prime}$, of the refractive index, $m=m^{\prime}+i m^{\prime \prime}$.

B.5 Fog aerosols, real and imaginary parts, $m^{\prime}$ and $m^{\prime \prime}$, of the refractive index, $m=m^{\prime}+i m^{\prime \prime}$ 105 


\section{List of Tables}

2.1 Haze aerosol models characteristic parameters: Bi-modal lognormal size distribution function $[13,22]$.

2.2 Fog aerosol models characteristic parameters: Modified Gamma size distribution function $[\mathbf{1 3}, \mathbf{2 2}]$.

2.3 Calculated optical parameters of fog atmospheric aerosol models for various meteorological range $(M)$ values

3.1 Optical properties of haze and fog atmospheric aerosol models in the visible, NIR, and MIR spectral wavelength bands $(0.39-5 \mu \mathrm{m})$.

3.2 The estimated noise equivalent spatial frequency, vnoise [cycle/rad], of the image-spectrum SNR for the atmospheric aerosol models, for each spectral band as shown in figure (3.6), and the corresponding threshold noise effective spatial resolution, $\theta_{t h}[\mathrm{rad}]$.

4.1 Optical properties of fog aerosol models in the visible wavelength band.

4.2 The estimated noise effective cutoff spatial frequency $v_{\text {noise }}[$ cycle/rad $]$ of the image spectrum SNR shown in figure (4.8), the corresponding threshold noise effective spatial resolution $\theta_{t h}[\mathrm{rad}]$, and the values of the restored spatial resolution $[m] ; \theta_{t h} R$ 


\section{Preface}

This dissertation is mainly comprised of three journal articles authored by my advisor, Prof. Michael Roggemann, my co-advisor, Dr. Durdu Guney, and myself. The first article [1] appears in Chapter 2 was published in the Journal of the Optical Society of America A (JOSA A), volume (31), issue (6), June 2014, as "Detailed effects of scattering and absorption by haze and aerosols in the atmosphere on the average point spread function of an imaging system". The content of Chapter 3 is sourced from "Estimating the image spectrum signal-to-noise ratio for imaging through scattering media". This paper was submitted to SPIE Optical Engineering. The third paper entitled "Reconstruction of images degraded by aerosol scattering and measurement noise" appears as submitted to SPIE Optical Engineering in Chapter 4. Prof. Roggemann is a contributing author for the three papers as he provided the main idea and helped me develop, write, edit, and review the papers. Dr. Guney contributed to the stage of reviewing and editing the three papers. As a first author, I performed all other contributing work and wrote and prepared the manuscript, also I developed a stand-alone Matlab code that characterizes the optical properties of the haze and fog atmospheric aerosol models that were analyzed throughout the whole thesis. Though the work and contributions presented in this work are my own, their completion and publication would not have been possible without my advisors feedback and recommendations for improvement. 
$\mathrm{x}$ viii 


\section{Acknowledgments}

First and foremost, thanks to God the merciful and compassionate. I would like to express my great indebtedness and gratitude to my advisor, Prof. Michael C. Roggemann. I feel extremely fortunate having been his advisee. To him, I am sincerely grateful for offering much of his precious time guiding me, revising and correcting many of shortcomings of this research. Many thanks to Dr. Durdu O. Guney, for his tremendous advices and generous reviewing to produce this work. Thanks also goes out to my committee for their time and helpful suggestion in improving my research. My wife also deserves a special recognition for supporting me at my hardest ever times, and for her endless and continuous encourage, assistance, and passion. Special thanks go to my brother Abdulaziz Alorf for his generosity and support. I would like to acknowledge my dept to Dr. Fawzy Eltohamy, Dr. Ibrahim Abdeldayem, and my family for encouraging and supporting me. Finally, I thank Michigan Technological University and the surrounding community, alumni, and staff who support this institution, for the opportunities they have created for me throughout my career and into the future. 


\section{Abstract}

Atmospheric scattering plays a crucial rule in degrading the performance of electrooptical imaging systems operating in the visible and infra-red spectral bands, and hence limits the quality of the acquired images, either through reduction of contrast or increase of image blur. The exact nature of light scattering by atmospheric media is highly complex and depends on the types, orientations, sizes and distributions of particles constituting these media, as well as wavelengths, polarization states and directions of the propagating radiation. Here we follow the common approach for solving imaging and propagation problems by treating the propagating light through atmospheric media as composed of two main components: a direct (unscattered), and a scattered component. In this work we developed a detailed model of the effects of absorption and scattering by haze and fog atmospheric aerosols on the optical radiation propagating from the object plane to an imaging system, based on the classical theory of EM scattering. This detailed model is then used to compute the average point spread function (PSF) of an imaging system which properly accounts for the effects of the diffraction, scattering, and the appropriate optical power level of both the direct and the scattered radiation arriving at the pupil of the imaging system. Also, the calculated PSF, properly weighted for the energy contributions of the direct and scattered components is used, in combination with a radiometric model, to estimate the average number of the direct and scattered photons detected at the sensor plane, which are then used to calculate the image spectrum signalto-noise ratio (SNR) in the visible near infra-red (NIR) and mid infra-red (MIR) spectral 
wavelength bands. Reconstruction of images degraded by atmospheric scattering and measurement noise is then performed, up to the limit imposed by the noise effective cutoff spatial frequency of the image spectrum SNR.

Key results of this research are as follows: A mathematical model based on Mie scattering theory for how scattering from aerosols affects the overall point spread function (PSF) of an imaging system was developed, coded in MATLAB, and demonstrated. This model along with radiometric theory, was used to predict the limiting resolution of an imaging system as a function of the optics, scattering environment, and measurement noise. Finally, image reconstruction algorithms were developed and demonstrated which mitigate the effects of scattering-induced blurring to within the limits imposed by noise. 


\section{Chapter 1}

\section{Introduction}

The Earth's atmosphere has significant effects on the propagation of electromagnetic (EM) radiation, and accordingly degrades the performance of electro-optical systems which are designed for civilian and military applications, particularly over long atmospheric paths. These effects are attributed to atmospheric turbulence, and to absorption and scattering of EM waves by atmospheric molecules and aerosols. The large-angle scattering, caused by atmospheric particulates whose diameters are small relative to the propagation wavelength, leads to attenuation of the direct signal and cause contrast reduction. Small-angle forward scattering, caused by large-sized aerosols, has the effect of broadening the angle at which the scattered light arrives at the receiver aperture compared to the unscattered light, and this light is spatially de-correlated from the unscattered light, even though both the scattered and unscattered light originated at the target, and hence causes image blur. Atmospheric aerosol scattering causes image blur in a different mechanism than that caused by turbulence. Atmospheric turbulence causes random aberrations which cause both short and long term blurring of the images, and line of sight jitter referred to as tilt errors, by virtue of light propagation through random index of refraction fluctuations caused by turbulence mixing of the air $[\mathbf{2}, \mathbf{3}]$. Hence, turbulence induced errors are experienced by all of the optical energy reaching the 
receiver aperture, whereas in the case of aerosol scattering some of the light arriving from the source is unscattered. On the other hand, aerosol scattering has the effect of light diffusion and broadening the angle at which the scattered light arrives at the receiver compared to the un-scattered light, hence scattering has the effect of de-correlating the light leaving the target from the unscattered light reaching the imaging system, and scattering has the effect of broadening the angle at which the scattered light arrives at the receiver compared to the unscattered light.

Our work is concerned with imaging through atmospheric scattering media, hence the degrading effect of turbulence on image formation will not be considered. The aim of this work is to investigate the degrading effects of light scattering and measurement noise on the images acquired by imaging systems, and to reconstruct these images based on image spectrum signal to noise ratio (SNR) considerations. We hope that this work will assist in a better understanding of the degrading effects of light scattering phenomena and provide a discipline towards image reconstruction of images acquired through atmospheric aerosols.

\subsection{Historic overview}

Scattering of light by atmospheric media has been one of the main topics of research in the atmospheric optics and astronomy communities. Most imaging-based applications such as surveillance, terrain classification and border security require accurate detection of image features. In the presence of atmospheric scattering, however, the images 
acquired by elecro-optical systems are drastically altered or degraded, and it is critical to understand the optical phenomena in order to reconstruct the degraded images. Historically, single scattering by a spherical particle was completely described by Mie theory, whereas multiple scattering was handled by two distinct theories; the analytical theory and the transport theory [4]. The analytical theory starts with Maxwell equations or the wave equation, introduces the scattering and absorption characteristics of particles, and obtains appropriate differential or integral equations for the statistical quantities such as variances and correlation functions that describe these characteristics. The mutual coherence function (MCF) that often characterizes the image quality, and from which the modulation transfer function (MTF) can be derived, is introduced through this theory $[\mathbf{4}$, 5]. Transport theory, on the other hand, doesn't start with the wave equation. It deals directly with the transfer of energy through a medium containing particles. The specific intensity (SI), which represents the time or space averaged power flow, is introduced through this theory [4]. It is possible to solve the equation of radiation transfer either numerically or analytically and to get an analytical expression for the atmospheric contribution to spatial blurring [6]. Monte Carlo method is the most general numerical technique used to calculate the effects of an arbitrary turbid atmosphere on optical systems [7-10]. Even though the starting points of the analytical theory and that of the radiative transfer theory are different, they are linked together through a fundamental relationship, where the mutual coherence function is a Fourier transform of the specific intensity [4]. In general, the exact nature of scattering is highly complex and depends on the types, orientations, sizes and distributions of particles constituting the media, as well as wavelengths, polarization states and directions of the incident light. The literature on 
this topic has been written over the past two centuries. Our major sources of background material that we used in our research are the works of H. C. van de Hulst [11], D. Deirmendjian [12], E.P. Shettle and R.W. Fenn [13], A. Ishimaru [4], and C. F. Bohren and D. R. Huffman [14], whose materials, serve as excellent reviews of prior work. Through the nineties of the previous century a debate was risen between researchers about the limiting effect of atmospheric scattering on the quality of images $[6,15-17]$. Kopeika proposed a model which was the first theoretical effort to predict how the radiation scattered by the atmosphere is detected by an imaging system [15]. Kopeika was the first to point out that the atmospheric scattering MTF that was predicted by many authors is not the one that is actually measured by an imaging system. Kopeika used the radiative transfer theory to define the term "practical instrumentation-based atmospheric aeroaol MTF", which accounts for the combined effects of the atmosphere and the different components of the imaging system, including the field of view, sensor dynamic range, and spatial frequency bandwidth of imaging system, on blurring the acquired images. On the other hand, Ben Dor presented another physical model that describes the effect of the radiation scattered by the aerosols and the molecules in the atmosphere on an imaging system [6]. This model explained how the field of view, the dynamic range, and other characteristics of the imaging system, as well as the turbidity of the atmosphere, affect the measured point spread function (PSF) of a distant point source. Ben Dor disagreed with Kopeika and mentioned that what is defined in Kopeika's paper as "practical aerosol MTF" represents the MTF of the imaging system combined with a small contribution of the system scattered radiation. Also, Ben Dor restricted the 
degrading effects due to scattering mainly to contrast reduction, except at extremely turbid atmosphere which may cause some image blur.

\subsection{Approach}

The approach taken in this research is now described. Throughout this work some important assumptions have been taken into consideration: first we assume that the scattered light has the same frequency; i.e. the same wavelength, as the incident light. A second assumption is that all the particles constituting the studied atmospheric media are spherical in shape, randomly distributed, and are independent from each other. Also, we follow the common approach for solving imaging and propagation problems by treating the propagating light through atmospheric media as composed of two main components: a direct (unscattered), and a scattered component. The thesis addresses the degrading effects of atmospheric scattering by three corner stones: First, we developed a detailed EM model of the effects of absorption and scattering by atmospheric aerosols on the optical radiation propagating from the object plane to an imaging system based on the classical theory of EM scattering. This detailed EM model is used to compute the average PSF of an imaging system which properly accounts for the effects of the diffraction, scattering, and the appropriate optical power level of both the unscattered and the scattered radiation arriving at the pupil of the imaging system. Since the scattered radiation is temporally and spatially de-correlated from the unscattered radiation we model the effects of the unscattered radiation, and the radiation scattered from the various species as additive in the image plane. Second, the image spectrum signal-to-noise ratio (SNR) provides a means of estimating the noise effective spatial resolution of an imaging 
system, and a means of estimating the highest spatial frequency which can be reconstructed with a post detection image reconstruction algorithm. In this thesis we extend previous work that has addressed the effects of aerosol scattering on the overall PSF to also account for the effects of measurement noise, and to then estimate the noise effective spatial resolution of the system, which accounts for scattering effects on the PSF and measurement noise in the detector. We use radiometric calculations to estimate the mean numbers of direct and scattered photons detected by an imaging system due to reflected radiation in the visible and near infra-red (NIR), and emitted radiation in mid infra-red (MIR) band, for a horizontal near-ground imaging scenario. Estimating the image spectrum SNR allows the calculation of the noise effective cutoff spatial frequency for images taken through fog aerosol media, hence emphasizes the degrading effect of fog aerosol models on the spatial resolution of imaging systems. Third, the problem of reconstructing images degraded by aerosol-induced blurring and measurement noise effects is examined using estimates of the overall PSF and image spectrum SNR considerations. Results of the reconstructions show that spatial resolution can be recovered in badly blurred images up to the limit imposed by the noise equivalent spatial frequency of the measurement.

\subsection{Summary of key results}

In this thesis, a detailed model of scattering and absorption by haze and aerosols in the 
atmosphere is used to compute the average PSF of an imaging system. A key result of this research is developing a method to quantify in details the significant effect of atmospheric scattering on the contrast and spatial resolution of images acquired by imaging systems, due to the increased level of the scattered radiation PSF and the reduced level of the direct radiation PSF upon increasing the atmospheric optical depth. Also, the calculation of the image spectrum SNR as a function of the direct and scattered components of the effective PSF for haze and fog aerosol model showed a reduction in the value of noise effective cutoff spatial frequency for images taken through fog aerosol media, hence emphasizes the degrading effect of fog aerosol models on the spatial resolution of imaging systems which operate in the visible, NIR, and MIR spectral bands. Our key contribution is the demonstration of a technique for predicting the resolution limit of an imaging system working in the presence of fog and haze as a function of the optical system, the scattering medium, and the signal level. Finally, results of reconstruction of images degraded by aerosol-induced blurring and measurement noise effects, which is examined using estimates of the overall PSF and image spectrum SNR considerations, show that spatial resolution can be recovered in badly blurred images up to the limit imposed by the noise effective cutoff spatial frequency of the measurement.

\subsection{Organization}

The remainder of the dissertation is comprised of the contents of three journal articles which have been either published or under review by the editors of SPIE Optical 
Engineering. Minor changes to formatting and language in the articles have been made where appropriate. Chapter 2 is derived from "Detailed effects of scattering and absorption by haze and aerosols in the atmosphere on the average point spread function of an imaging system" [1], which was published in the Journal of the Optical Society of America A (JOSA A), Vol. 31, No. 6, June 2014. This paper is a detailed overview of light scattering by haze and fog aerosol models, in which we investigated several topics: Mie scattering theory was used to model the optical properties of a single spherical particle, also the physical parameters of various haze and fog atmospheric aerosol models, including their particle compositions and size distribution functions, were incorporated to calculate the optical properties of these atmospheric media; also we used this detailed model to compute the average PSF of the atmosphere/telescope system, which is the basic physical function that describes the spatial response of an imaging system.

The content of Chapter 3 has been appeared as "Estimating the image spectrum signal-tonoise ratio for imaging through scattering media", which have been submitted to SPIE Optical Engineering and is currently under review. This paper used the effective PSF calculations, presented in the first paper, in combination with a radiometric model for estimating the average number of photons detected by an imaging system from an extended target, for a horizontal near-ground imaging scenario, upon propagation through various haze and fog aerosol models. The model considers both the reflected radiation from an extended target in the visible and NIR bands, and the emitted radiation from the target in the MIR band. The mean numbers of direct and scattered photons detected by an imaging system are then estimated based on the radiometric model and PSF calculations. 
Finally, we adopted an approach for estimating the image spectrum SNR, which provides a means of estimating the noise equivalent spatial frequency of an imaging system, and a means of estimating the highest spatial frequency which can be reconstructed with a post detection image reconstruction algorithm.

Chapter 4 has been appeared as "Reconstruction of images degraded by aerosol scattering and measurement noise", which have been submitted to SPIE Optical Engineering. In this paper we examine the problem of reconstructing images degraded by aerosol-induced blur and measurement noise using estimates of the effective PSF and image spectrum SNR considerations, that were investigated in chapter 3 . Representative images of a spoke target acquired under various conditions of scattering and photon flux levels were simulated and are shown in this paper. Reconstruction of the degraded images is then performed using a Wiener filter and a constrained least squares filter.

Chapter 5 summarizes the conclusions and the findings in this body of work, and provides guidance for future research. 


\title{
Chapter 2
}

\section{Detailed Effects of Scattering and}

\section{Absorption by Haze and Aerosols in The}

\author{
Atmosphere on The Average Point \\ Spread Function of an Imaging System
}

1

1010

1 The material contained in this chapter was previously published in the Journal of the Optical Society of America A, JOSA A / Vol. 31, No. 6 / June 2014 [1]. See Appendix $\mathrm{C}$ for documentation of permission to republish this material. 


\subsection{Introduction}

The propagation of electro-magnetic (EM) radiation within the Earth's atmosphere depends on the wavelength of the propagating radiation, $\lambda$, and on the nature of the medium being traversed. This medium consists of various molecular species and aerosol particles whose composition and density are the determining factors in the amount of radiation detected by an electro-optical imaging system. EM fields propagating through the atmosphere are either attenuated or blurred. Attenuation is attributed to absorption and large angle scattering (Rayleigh scattering) caused by atmospheric molecules which have small size compared to the propagation wavelength, $r<<\lambda$, where $r$ is the particulate radius. Blurring is attributed to optical turbulence and small angle forward scattering (Mie scattering) caused by large sized aerosols, $r \geq \lambda$, which is known as the adjacency effect since photons are imaged in pixels adjacent to those in which they ought

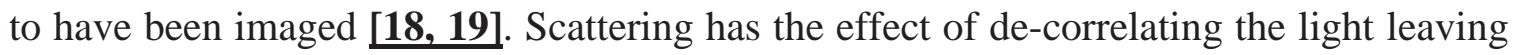
the target from the unscattered light reaching the imaging system, i.e., the scattered and direct radiation received at the imaging system entrance pupil, after propagation through a scattering medium, are uncorrelated. Also, scattering has the effect of broadening the angle at which the scattered light arrives at the receiver compared to the unscattered light. In this paper, the combined effects of the refractive index and the particle size on the optical properties of a single spherical particle, including the EM cross sections, single scattering albedo, scattering phase function, and asymmetry parameter, will be outlined in the second section using Mie scattering theory (Appendix A). The third section investigates in details various haze and fog atmospheric aerosol models; where the 
physical parameters of these aerosol models, including their particle compositions and size distribution functions, are utilized to calculate their optical properties. In the fourth section we used this detailed model to compute the average point spread function (PSF), which is the basic physical function that describes the spatial response of an imaging system, that properly accounts for the effects of the diffraction, scattering, and the appropriate optical power level of both the unscattered and the scattered radiation arriving at the pupil of the imaging system. Since the scattered radiation is temporally and spatially de-correlated from the unscattered radiation we model the effects of the unscattered radiation, and the radiation scattered from the various atmospheric species as additive in the image plane $[\mathbf{6}, \mathbf{2 0}]$. Conclusions are given in the fifth section. Although the different aspects of our model have been studied independently by other researchers, a comprehensive model, which links these different aspects in a coherent picture to explain how the characteristics of the EM waves propagating in Earth's atmosphere play a role in the ultimate performance of an imaging system, has not been studied, to the best of our knowledge. Our model links the detailed EM modeling of scattering and absorption phenomena due to a single spherical particle to the atmospheric aerosol models involving many particles with various size and composition, which in turn are linked to the effects on the detection capability of the imaging system that is expressed by PSF analysis. 


\subsection{Optical properties of a single spherical particle}

In this section, Mie scattering theory will be used to explore the combined effects of the size of a homogeneous spherical particle, with radius $r$, and refractive index relative to the refractive index of the ambient medium, $m=m^{\prime}+i m^{\prime \prime}$, on the optical properties of the particle including EM cross sections, single scattering albedo, scattering phase function, and asymmetry parameter. All the plots given in sections (2.2) and (2.3) are constructed using a stand-alone Matlab code, and are shown here to be consistent with the results obtained by Bohren and Huffman [14], and the results given by Shettle and Fenn [13].

\subsubsection{EM efficiency factors}

The EM cross sections of a single scattering particle, measured in $\left[\mathrm{m}^{2}\right]$, include the absorption cross section $\sigma_{a b s}$, scattering cross section $\sigma_{s c a}$, and extinction cross section $\sigma_{e x t}$ EM cross sections describe the interaction of a particle with an incident field. $\sigma_{a b s}$ represents the cross section of a particle which would correspond to the total power absorbed by the particle, whereas $\sigma_{\text {sca }}$ represents the cross section of a particle which would produce the total observed scattered power at all angles surrounding the particle. The sum of $\sigma_{a b s}$ and $\sigma_{s c a}$ gives the total cross section or extinction cross section; $\sigma_{e x t}=\sigma_{a b s}+\sigma_{s c a}$. EM cross sections normalized to the particle's geometrical cross section $\pi a^{2}$ are termed the efficiency factors, $Q_{i}[\mathbf{1 4}]$ 


$$
Q_{i}=\frac{\sigma_{i}}{\pi a^{2}}
$$

where $i$ stands for absorption $(i=a b s)$, scattering $(i=s c a)$, or extinction $(i=e x t)$. For a spherical particle with size parameter $x$, and complex refractive index $m$, Mie coefficients $a_{n}$ and $b_{n}$ are used to calculate the efficiency factors $[4,14]$,

$$
\begin{gathered}
Q_{s c a}=\frac{2}{x^{2}} \sum_{n=1}^{\infty}(2 n+1)\left(\left|a_{n}\right|^{2}+\left|b_{n}\right|^{2}\right) \\
Q_{\text {ext }}=\frac{2}{x^{2}} \sum_{n=1}^{\infty}(2 n+1) \operatorname{Re}\left(a_{n}+b_{n}\right) \\
Q_{a b s}=Q_{\text {ext }}-Q_{\text {sca }}
\end{gathered}
$$

Figures (2.1) and (2.2) show plots of the efficiency factors $Q_{e x t}, Q_{s c a}$ and $Q_{a b s}$ versus size parameter $x$. The propagation wavelength is that for the visible band; $\lambda=0.55[\mu \mathrm{m}]$, and the particle radius is allowed to vary. Figure (2.1) shows the effect of the imaginary part of the complex refractive index, $m^{\prime \prime}$, on the values of $Q_{e x t}, Q_{s c a}$ and $Q_{a b s}$ for a spherical particle having a fixed $m^{\prime}$ value and various values of $m^{\prime \prime}$. 

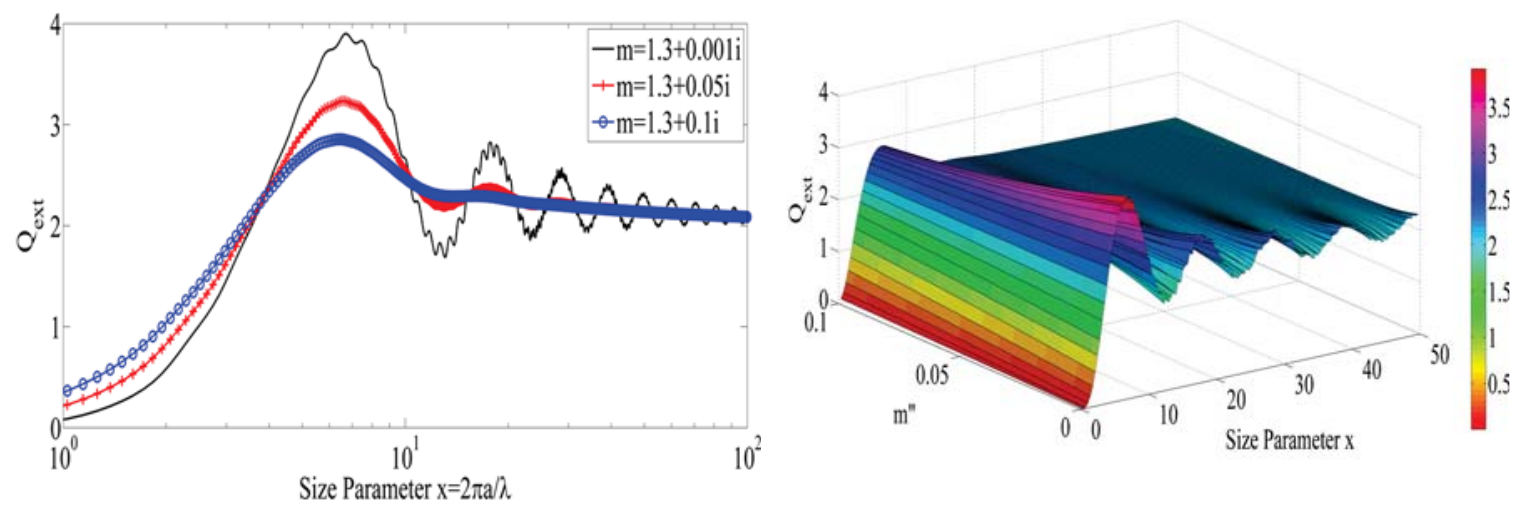

(a)
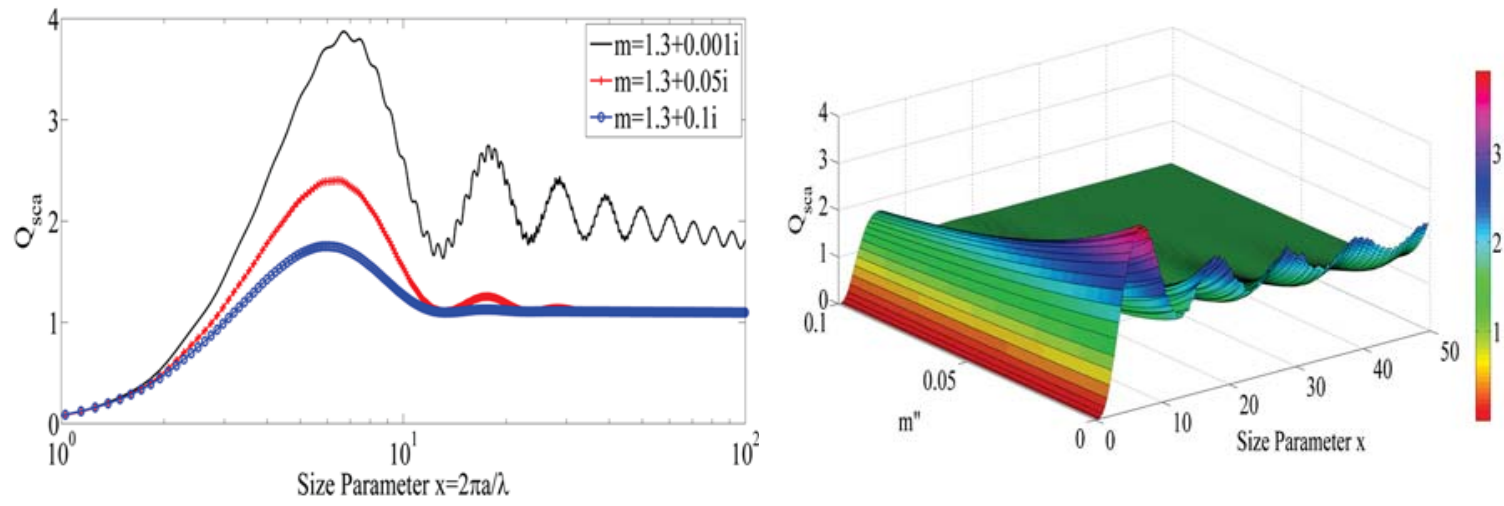

(b)
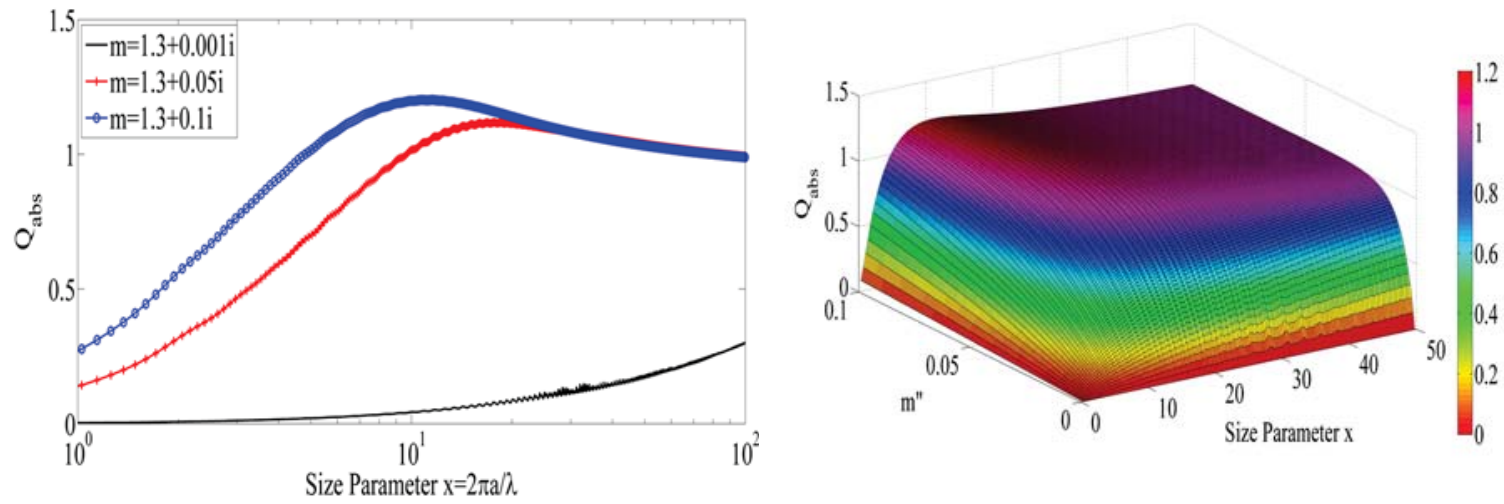

(c)

Figure 2.1 Extinction efficiency factor, $Q_{e x t}$, Scattering efficiency factor, $Q_{s c a}$, and Absorption efficiency factor, $Q_{a b s}$, versus size parameter, $x$, for a spherical particle with $m^{\prime}=1.33$, and various $m^{\prime \prime}$ values, at visible wavelength.

(a) $Q_{\text {ext }}$, (b) $Q_{s c a}$, (c) $Q_{a b s}$. 
Figure (2.1.a) reveals several features for $Q_{\text {ext }}$ corresponding to the values of $x$ and $\mathrm{m}^{\prime \prime}$. For particles in the Rayleigh regime, $x<<2 \pi$, $Q_{\text {ext }}$ curves increase monotonically, whereas for particles in the Mie regime, $x \geq 2 \pi, Q_{\text {ext }}$ curves have a series of regularly spaced broad maxima and minima (series of peaks and valleys) called the interference structure which is oscillating approximately about the value 2, and another irregular fine structure called ripple structure [14]. The interference structure is attributed to the interference between the un-deviated beam and the central ray through a sphere. As $m$ " values decrease, these structures are more apparent throughout the Mie scattering regime. Whenever $m^{\prime \prime} x \geq 1$, no appreciable light penetrates through the particle and accordingly both the interference and ripple structures are strongly damped. Also, it is apparent that $Q_{\text {ext }}$ reaches its maximum value for a particle radius equivalent to propagation wavelength $(r=\lambda)$. These features appear also at the $Q_{s c a}$ plots given in figure (2.1.b), where for each curve, as $m^{\prime \prime} x \geq 1$; the interference and ripple patterns fade, and $Q_{\text {sca }}$ reaches its maximum value for $r=\lambda$. In Figure (2.1.c), for any given value of $x$; as $m$ " increases, $Q_{a b s}$ also increases indicating increased radiation absorption upon increasing the imaginary part of the complex refractive index, also the maximum value of $Q_{a b s}$ shifts toward smaller particles. Figure (2.2) shows the effect of the real part $m^{\prime}$ of the complex refractive index on the values of $Q_{e x t}, Q_{s c a}$ and $Q_{a b s}$ for a spherical particle having a fixed $m^{\prime \prime}$ value and various values of $m^{\prime}$. Hence, the particle composition described by its complex refractive index contributes to radiation extinction either through scattering which depends on the value of the real part of the refractive index, or through absorption which depends on the value of the imaginary part of the refractive index. 

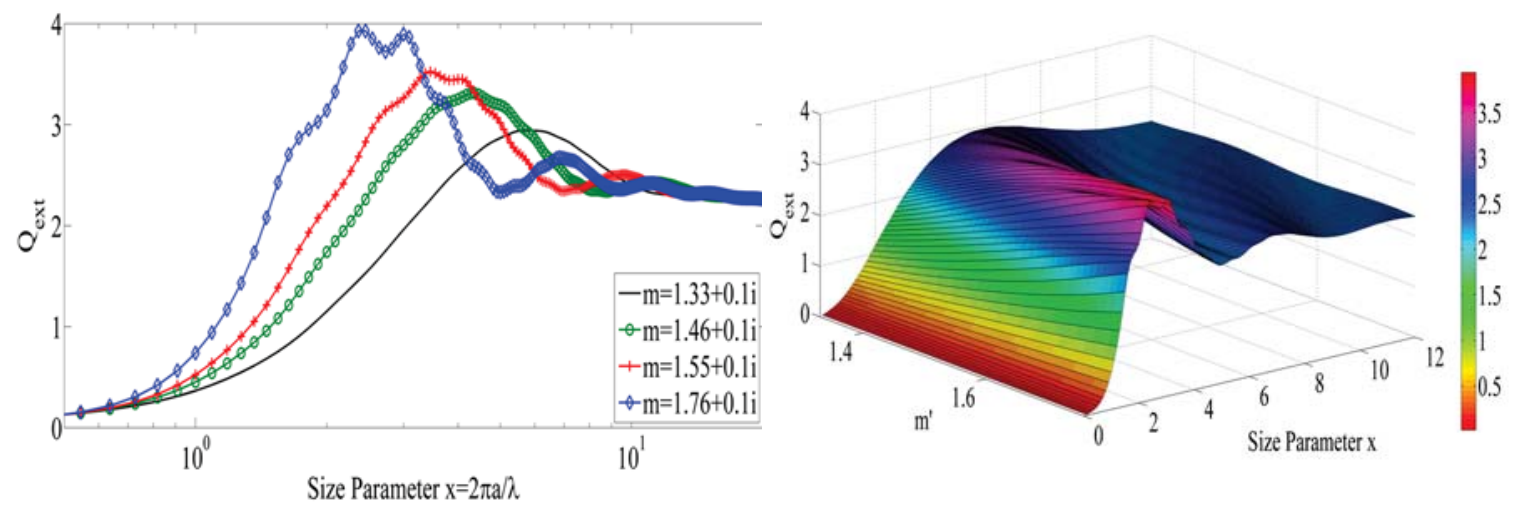

(a)
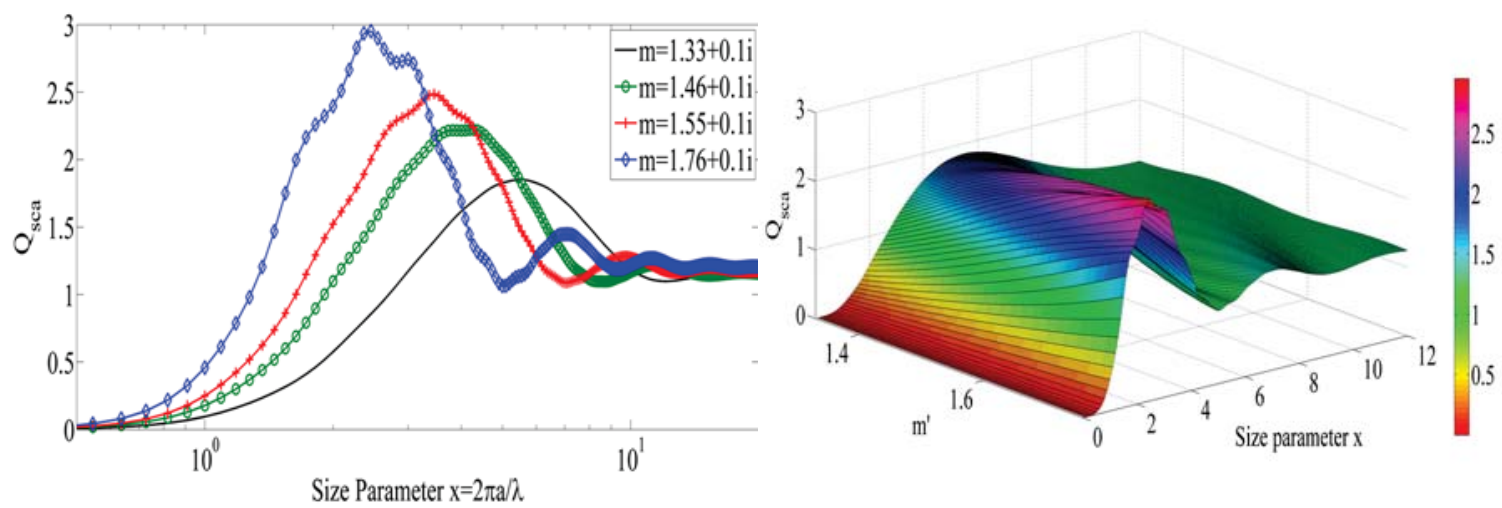

(b)
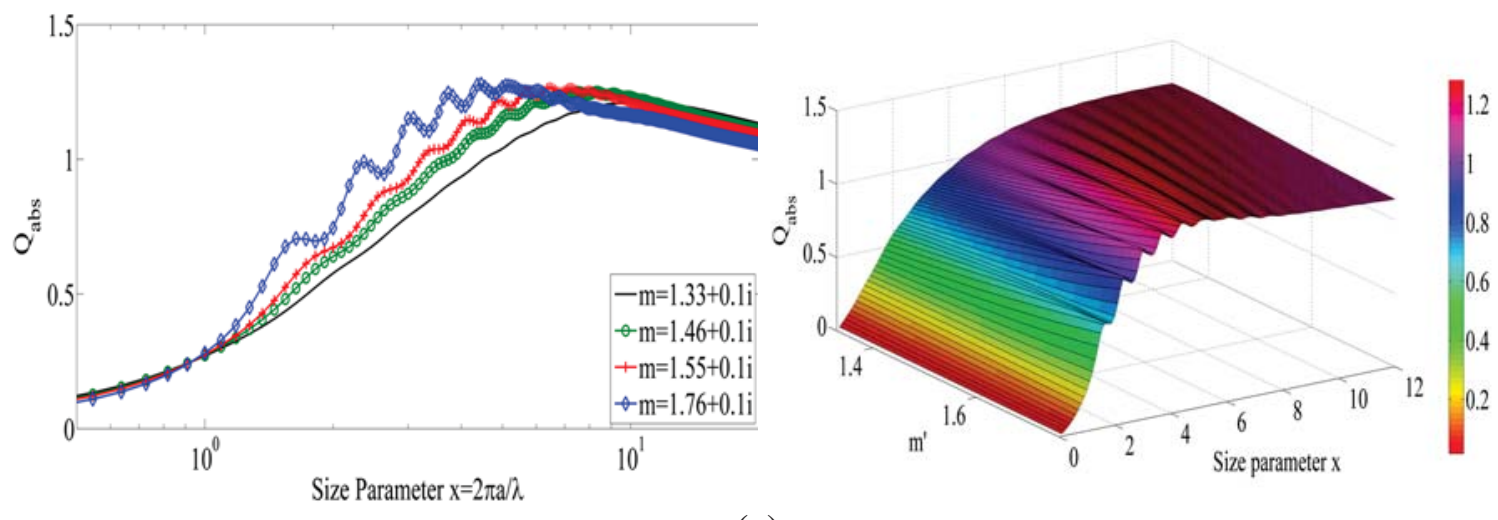

(c)

Figure 2.2 Extinction efficiency factor, $Q_{e x t}$, Scattering efficiency factor, $Q_{\text {sca }}$, and Absorption efficiency factor, $Q_{a b s}$, versus size parameter, $x$, for a spherical particle with $m^{\prime \prime}=0.1$, and various $m^{\prime}$ values, at visible wavelength.

(a) $Q_{e x t}$, (b) $Q_{s c a}$, (c) $Q_{a b s}$. 
A common feature given by figures (2.1) and (2.2) is that for very large $x$ values, $Q_{s c a}$ and $Q_{a b s}$ approach their geometrical optics limit of 1, whereas $Q_{e x t}$ approaches its geometrical optics limit of 2 .

\subsubsection{Single Scattering Albedo}

At all wavelengths, aerosols generally scatter radiation into various angles out of the lineof-sight between the radiation source and the imaging system. The single scattering albedo $\omega_{o}$ indicates the probability that a photon intercepted by a particle will be scattered rather than being absorbed, and is defined as the ratio of the particle scattering cross section to the extinction cross section [14],

$$
\omega_{o}=\frac{\sigma_{s c a}}{\sigma_{e x t}}
$$

$\omega_{o}$ is dimensionless with range $0 \leq \omega_{o} \leq 1$, for pure scattering, $\omega_{0}=1$. Figure (2.3) shows a plot of $\omega_{o}$ as a function of particle size parameter. Figure (2.3.a) shows the effect of varying $m^{\prime \prime}$ on $\omega_{o}$, for a spherical particle with fixed value of $m^{\prime}$. It is noticed that for a certain $x$ value; increasing $m^{\prime \prime}$ leads to a reduced $\omega_{o}$, indicating decreased scattering upon increased absorption. Figure (2.3.b) shows the effect of varying $m^{\prime}$ on $\omega_{o}$, for a spherical particle with fixed value of $m^{\prime \prime}$. 

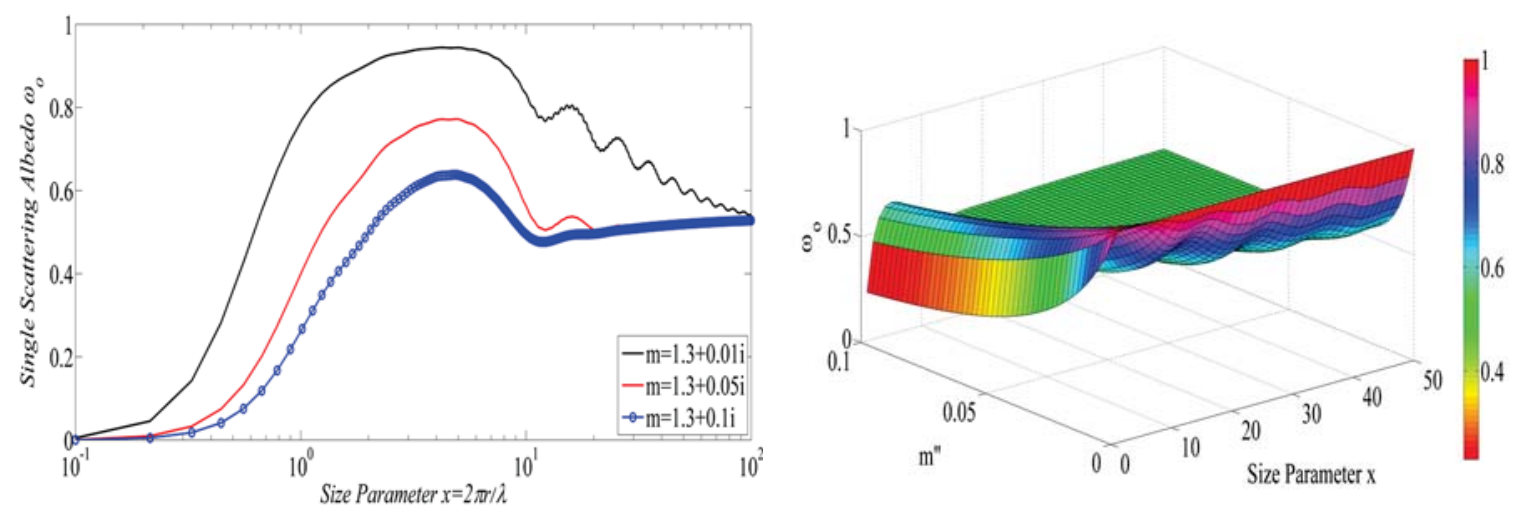

(a)
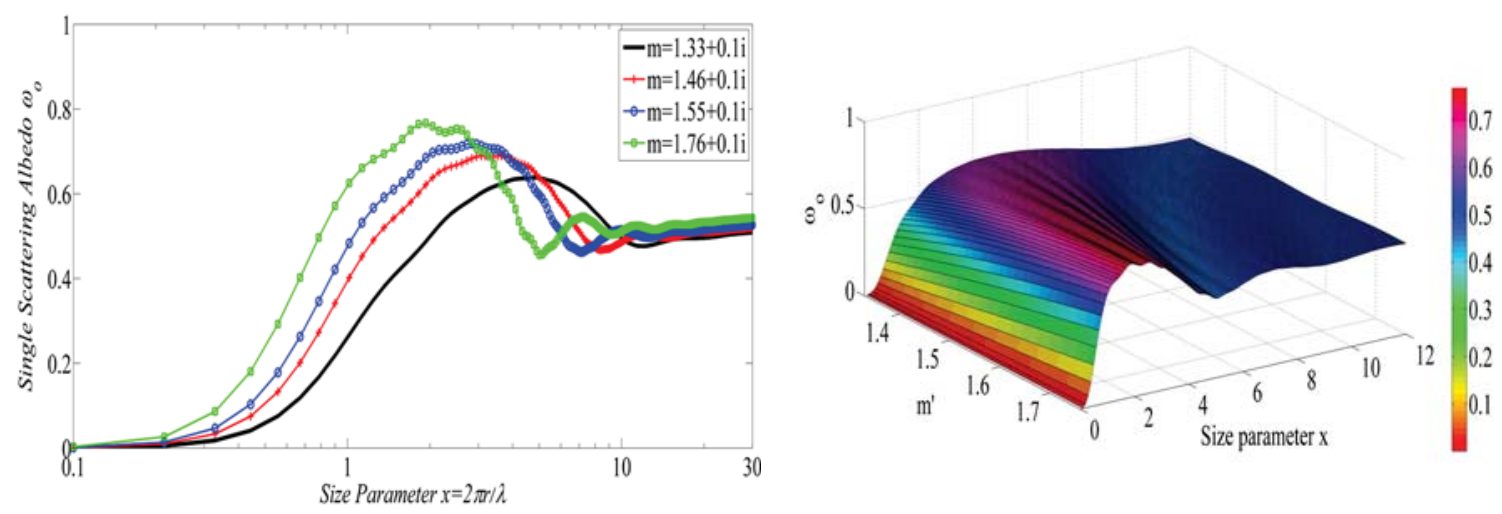

(b)

Figure 2.3 Single scattering albedo, $\omega_{o}$, versus size parameter, $x$, at visible wavelength, for (a) different values of the imaginary part of refractive index $m^{\prime \prime}$, and (b) different values of the real part of refractive index $m^{\prime}$.

\subsubsection{Scattering Phase Function}

The angular probability distribution of scattering radiation, according to a direction relative to the incident radiation, defines the scattering phase function $P(\theta)$, and is given by [14], 


$$
P(\theta)=\frac{\left|S_{1}(\theta)\right|^{2}+\left|S_{2}(\theta)\right|^{2}}{2 \pi x^{2} Q_{\text {sca }}}
$$

There is a strong dependence of forward scattering on particle size. Figure (2.4) shows the visible Mie scattering phase functions for differently sized spherical particles. It is clear that the narrow peaked phase function of the large sized particle will give rise to more forward scattered radiation than the widely peaked phase function of the small sized particle. Therefore, as $x$ increases the scattering phase function becomes more narrowpeaked towards small scattering angles indicating predominance of scattering in the forward direction for large sized particles.

\subsubsection{Asymmetry Parameter}

The asymmetry parameter $g$ is defined as the average cosine of the scattering angle [14].

$$
g=\langle\cos \theta\rangle=\int_{4 \pi} P(\theta) \cos \theta d \Omega
$$

$g$ is dimensionless with range $-1 \leq g \leq 1$. If the particle scatters more light toward the forward direction $(\theta=0), g$ is positive; if the particle scatters more light toward the backward direction $(\theta=180), g$ is negative. If $g=0$, then the light has the same probability of scattering in any direction; i.e., isotropic scattering. For highly peaked 


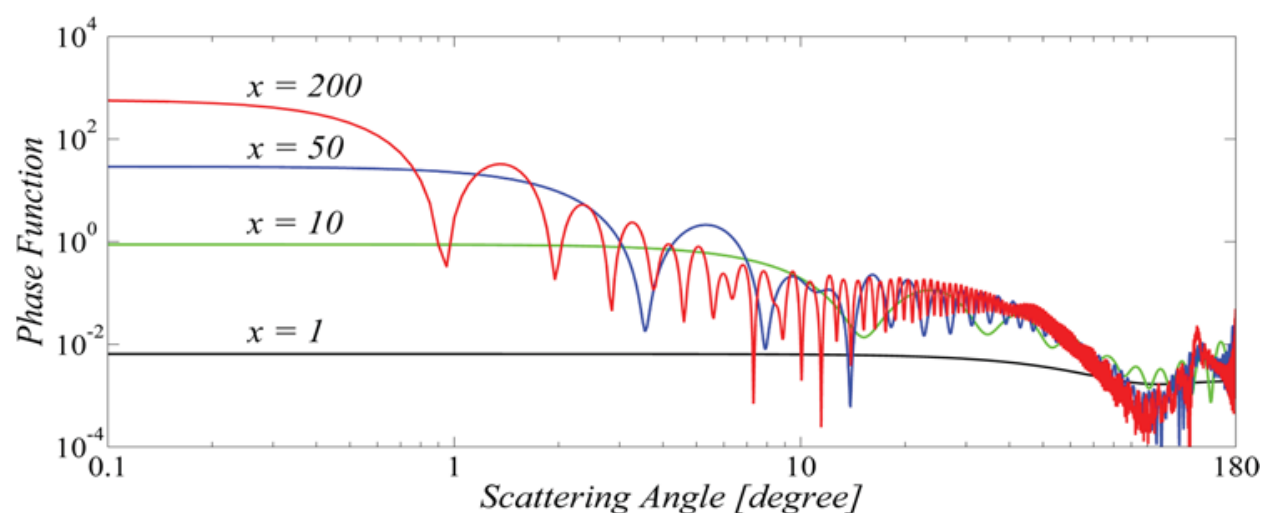

Figure 2.4 Single-scattering phase function, $P(\theta)$, for differently sized particles with refractive index $m=1.33+\mathrm{i} 10^{-8}$, at visible wavelength.

phase functions that is due to large sized particles, $g$ approaches its maximum value of 1 . The asymmetry parameter may be calculated by [21],

$$
g=\frac{4}{x^{2} Q_{\text {sca }}} \sum_{n=1}^{\infty}\left[\frac{n(n+2)}{n+1} \operatorname{Re}\left(a_{n} a_{n+1}^{*}+b_{n} b_{n+1}^{*}\right)+\frac{2 n+1}{n(n+1)} \operatorname{Re}\left(a_{n} b_{n}^{*}\right)\right]
$$

Figure (2.5) shows a plot of the asymmetry parameter as a function of particle size parameter. For particles in the Rayleigh regime, $g=0$ indicating an isotropic scattering. Figure (2.5.a) shows the effect of varying $m^{\prime \prime}$ on $g$. For particles in the Mie regime, at any given value of $x$, as $m^{\prime \prime}$ increases $g$ also increases indicating a considerable increased scattering in the forward direction. Also, as $m^{\prime \prime}$ decreases, $g$ shows the interference and ripple structures (that was previously discussed), again both structures fade for $m " x \geq 1$. As $x$ continues increasing, $g$ approaches a value of 1 indicating more forward scattering for large sized particles. Figure (2.5.b) shows the effect of varying $m^{\prime}$ on $g$ values; in some part of Rayleigh scattering regime, increasing $m^{\prime}$ corresponds to negative $g$ values, 
$-1 \leq g \leq 0$, indicating a backward scattering. Hence, the particle size and the real part of the refractive index contribute to the scattering direction either in the forward direction or backward direction.
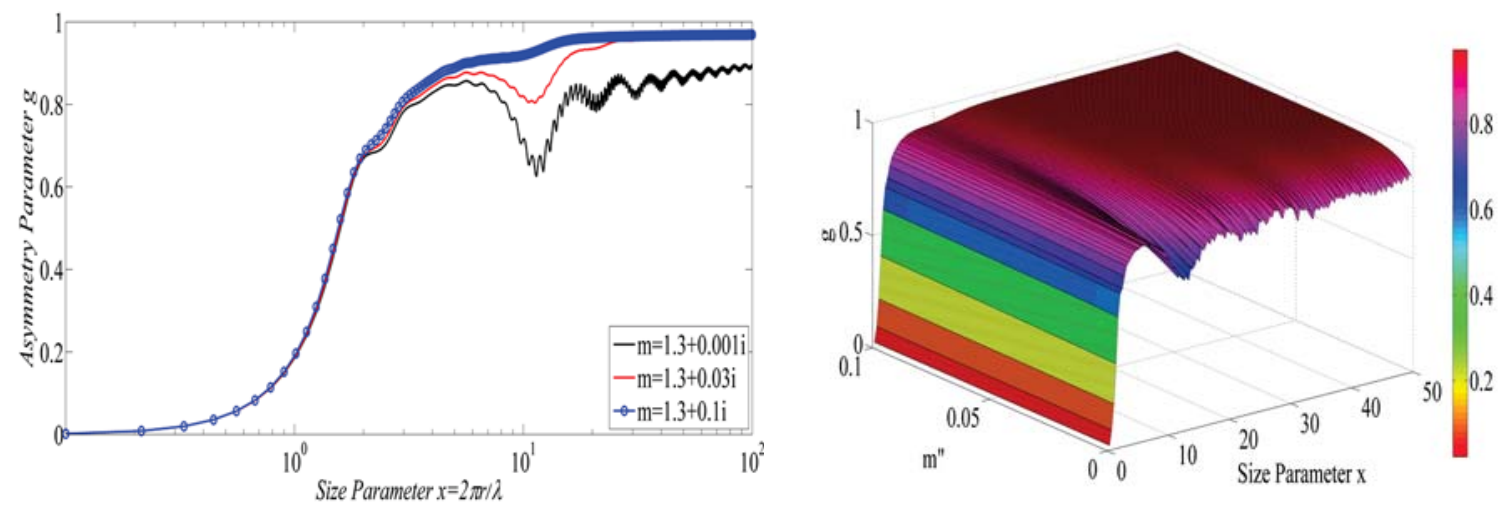

(a)
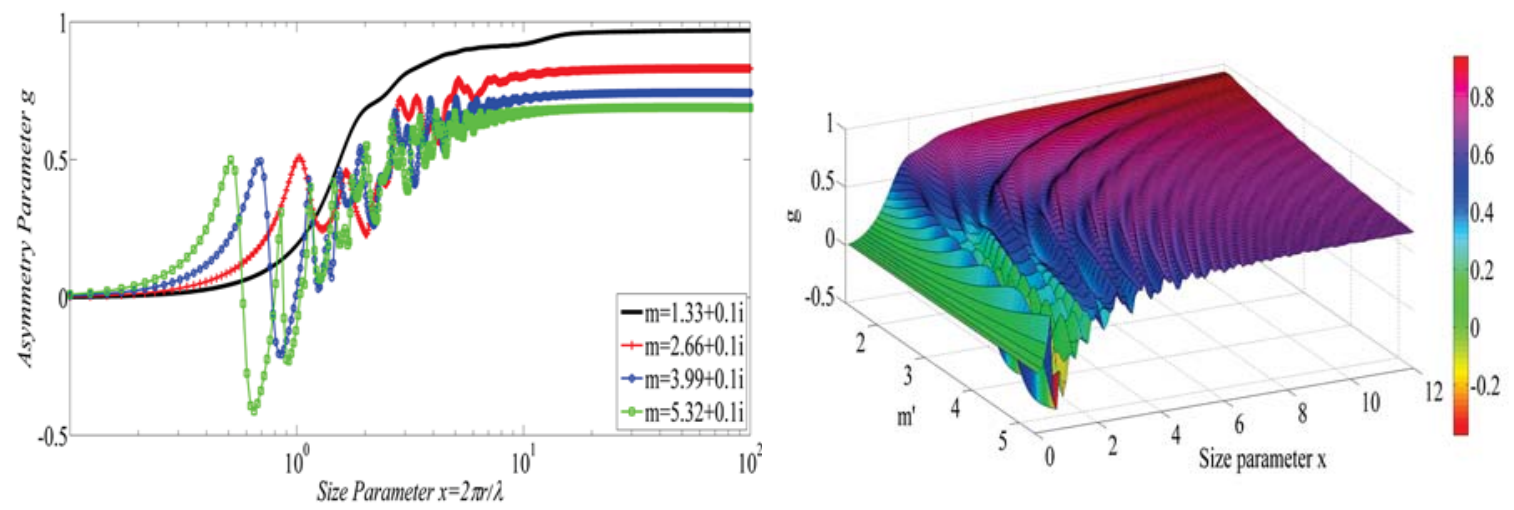

(b)

Figure 2.5 Asymmetry parameter, $g$, versus particle size parameter, $x$, at visible wavelength, for (a) different values of the imaginary part of refractive index $\mathrm{m}^{\prime \prime}$, and (b) different values of the real part of refractive index $m^{\prime}$. 


\subsection{Atmospheric aerosol models}

Aerosol modeling has two goals: accurate representation of the aerosol distribution (density) by size distribution functions, and representing the aerosol composition by spectral refractive index (Appendix B). The second goal is the accurate calculation of the optical properties of the whole medium including the atmospheric optical depth, EM attenuation coefficients, total albedo, scattering phase function, and asymmetry parameter.

\subsubsection{Particle size distribution function}

Aerosol particle size distribution functions, $n(r)$, used to model haze and fog aerosols, include lognormal, and modified gamma distributions, respectively [11, 13]. The lognormal distribution, used for smoke, dust, rural, urban, maritime, tropospheric, desert, and the Navy aerosol models, is given by $[\mathbf{1 2 , 1 3 , 2 2 - 2 4 ]}$,

$$
n(r)=\sum_{i=1}^{k}\left[\frac{N_{i}}{\sqrt{2 \pi} \ln (10) r \sigma_{i}}\right] \exp \left[-\frac{\left(\log _{10} r-\log _{10} r_{g i}\right)^{2}}{2 \sigma_{i}^{2}}\right]
$$

where $N_{i}$ is the total number of particles per unit volume $\left[\mathrm{m}^{-3}\right]$ for the $\mathrm{i}^{\text {th }}$ mode, $r_{g i}$ is the mode radius $[\mu m]$, and $\sigma_{i}$ is the mode standard deviation. The modified Gamma distribution, used to model fog, is given by $[\mathbf{1 2}, \mathbf{1 3}, \mathbf{2 2}]$, 


$$
n(r)=A r^{\alpha} \exp \left(-B r^{\gamma}\right)
$$

where $A, B, \alpha$ and $\gamma$ are fit coefficients of the distribution.

\subsubsection{Atmospheric optical properties}

The optical properties of an atmospheric aerosol model, including the atmospheric optical depth (AOD), the total albedo, the scattering phase function, and the asymmetry parameter, define the scattering and absorption behavior of the medium as a whole. The AOD, $\tau$ [dimensionless], expresses the power density removed from a propagating wave through the atmosphere upon scattering or absorption [25],

$$
\tau(\lambda)=\int k_{e x t}(\lambda, z) d z
$$

where $z$ is the atmospheric path length $[m], k_{\text {ext }}$ is the atmospheric extinction coefficient $\left[\mathrm{m}^{-1}\right]$, which represents the relevant cumulative extinction cross sections of all particles in a unit volume of air [6],

$$
k_{\text {ext }}(\lambda)=k_{s c a}(\lambda)+k_{a b s}(\lambda)
$$


where $k_{s c a}$ and $k_{a b s}$ are the scattering and absorption coefficients $\left[\mathrm{m}^{-1}\right]$, respectively. $k_{\text {ext }}(\lambda), k_{s c a}(\lambda)$, and $k_{a b s}(\lambda)$ are given by [26],

$$
k_{e x t}(\lambda)=\int_{0}^{\infty} \sigma_{e x t}(\lambda, r) n(r) d r
$$

$$
k_{s c a}(\lambda)=\int_{0}^{\infty} \sigma_{s c a}(\lambda, r) n(r) d r
$$

$$
k_{a b s}(\lambda)=\int_{0}^{\infty} \sigma_{a b s}(\lambda, r) n(r) d r
$$

The total albedo $\omega_{o}^{t}(\lambda)$ is given by [26],

$$
\omega_{o}^{t}(\lambda)=\frac{k_{s c a}(\lambda)}{k_{e x t}(\lambda)}
$$

and the scattering phase function $P(\lambda, \theta)$ is given by [26],

$$
P(\lambda, \theta)=\frac{1}{k_{s c a}(\lambda)} \int_{0}^{\infty} \sigma_{s c a}(\lambda, r) P(\lambda, r, \theta) n(r) d r
$$


whereas the asymmetry parameter $g(\lambda)$ is given by [26],

$$
g(\lambda)=\frac{1}{k_{s c a}(\lambda)} \int_{0}^{\infty} \sigma_{s c a}(\lambda, r) g(\lambda, r) n(r) d r
$$

\subsubsection{Haze and fog atmospheric aerosol models}

The data on the size distribution functions and the spectral refractive indices for atmospheric aerosols, in the wavelength range $\lambda=0.2-40[\mu \mathrm{m}]$, are used to calculate the optical properties of the investigated aerosol models $[13,22]$. These properties are required to make estimates of the atmospheric transmittance and angular light scattering distribution, which contribute to the PSF of an imaging system. It is assumed that these models contain homogeneous spherical particles that are randomly distributed. These models include four haze aerosol models: maritime, urban, rural, and tropospheric haze; and four fog aerosol models: heavy advection, moderate advection, heavy radiation, and moderate radiation fog. Tables (2.1) and (2.2) list the characteristic parameters corresponding to various relative humidity $(R H)$, and meteorological range $(M)$ values $[\mathrm{m}]$, of the haze aerosol models which are characterized by a bi-modal lognormal distribution function (in accordance with Eq. 2.9), and the fog aerosol models which are characterized by a modified Gamma size distribution function (in accordance with Eq. 2.10). Plots of the size distribution function, $n(r)$, for the haze aerosol models, and for

the fog aerosol models, listed in tables (2.1) and (2.2), are shown in figures (2.6) and (2.7), respectively. Plots of $k_{\text {ext }}(\lambda), k_{a b s}(\lambda), k_{s c a}(\lambda)$ for the haze aerosol models, and for the 
Table 2.1 Haze aerosol models characteristic parameters: Bi-modal lognormal size distribution function [13, 22].

\begin{tabular}{ccccc}
\hline \multirow{2}{*}{$\begin{array}{c}\text { Size Distribution } \\
\text { Parameters }\end{array}$} & $\begin{array}{c}\text { Maritime } \\
\text { haze }\end{array}$ & $\begin{array}{c}\text { Urban } \\
\text { haze }\end{array}$ & $\begin{array}{c}\text { Rural } \\
\text { haze }\end{array}$ & $\begin{array}{c}\text { Tropospheric } \\
\text { haze }\end{array}$ \\
\cline { 2 - 5 } & 2 & 5 & 10 & 25 \\
\hline$M[\mathrm{~km}]$ & 90 & 80 & 70 & 50 \\
$R H(\%)$ & 0.03884 & 0.03514 & 0.02846 & 0.02748 \\
$r_{g 1}[\mu \mathrm{m}]$ & 0.35 & 0.35 & 0.35 & 0.35 \\
$\sigma_{1}$ & 27993 & 42110 & 34680 & 17060 \\
$N_{I}\left[\mathrm{~cm}{ }^{-3}\right]$ & 0.3803 & 0.5805 & 0.4571 & - \\
$r_{g 2}[\mu \mathrm{m}]$ & 0.4 & 0.4 & 0.4 & - \\
$\sigma_{2}$ & 261 & 7.8 & 6.4 & - \\
$N_{2}\left[\mathrm{~cm}^{-3}\right]$ & & &
\end{tabular}

Table 2.2 Fog aerosol models characteristic parameters: Modified Gamma size distribution function [13,22].

\begin{tabular}{cccccc}
\hline & \multicolumn{5}{c}{ Size distribution parameters } \\
\cline { 2 - 6 } Fog Aerosol Models & $M[\mathrm{~km}]$ & $\mathrm{A}$ & $\mathrm{B}$ & $\alpha$ & $\gamma$ \\
\hline Heavy Advection & 0.136 & 0.027 & 0.3 & 3 & 1 \\
Moderate Advection & 0.211 & 0.06592 & 0.375 & 3 & 1 \\
Heavy Radiation & 0.235 & 2.37305 & 1.5 & 6 & 1 \\
Moderate Radiation & 0.453 & 607.5 & 3 & 6 & 1 \\
\hline
\end{tabular}

fog aerosol models are shown in figures (2.8) and (2.9), respectively. Figure (2.10) shows the scattering phase function for tropospheric haze, moderate radiation fog, and heavy advection fog aerosol models. It is clear that the narrow peaked phase function of the heavy advection fog aerosol model, which is due to its relatively large particles size, will give rise to a more forward scattered radiation than the wide peaked phase functions of the moderate radiation fog and tropospheric haze aerosol models. It is emphasized at this 
point that the significance of extinction of a propagating radiation by an atmospheric aerosol model, is greatly dependent on the size distribution function, the aerosols composition of the model, and the propagation wavelength. Also, increased $k_{e x t}$ values, that lead to an increased AOD, indicate an increased amount of scattered radiation reaching the imaging system which will affect the acquired image significantly.

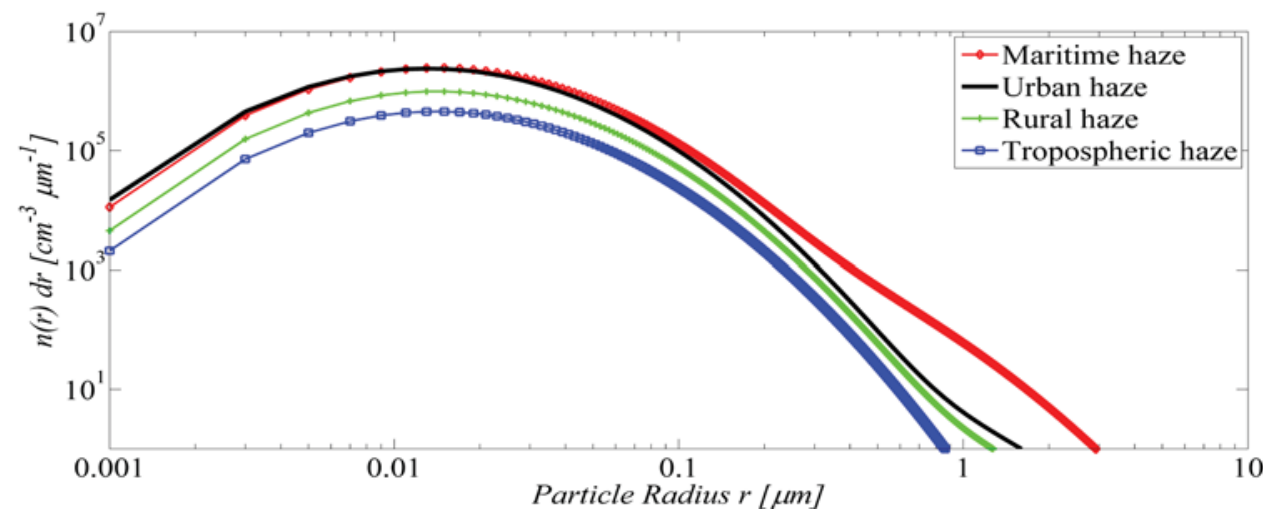

Figure 2.6 Bi-modal lognormal size distribution function for haze aerosol models in accordance with data listed in table (2.1).

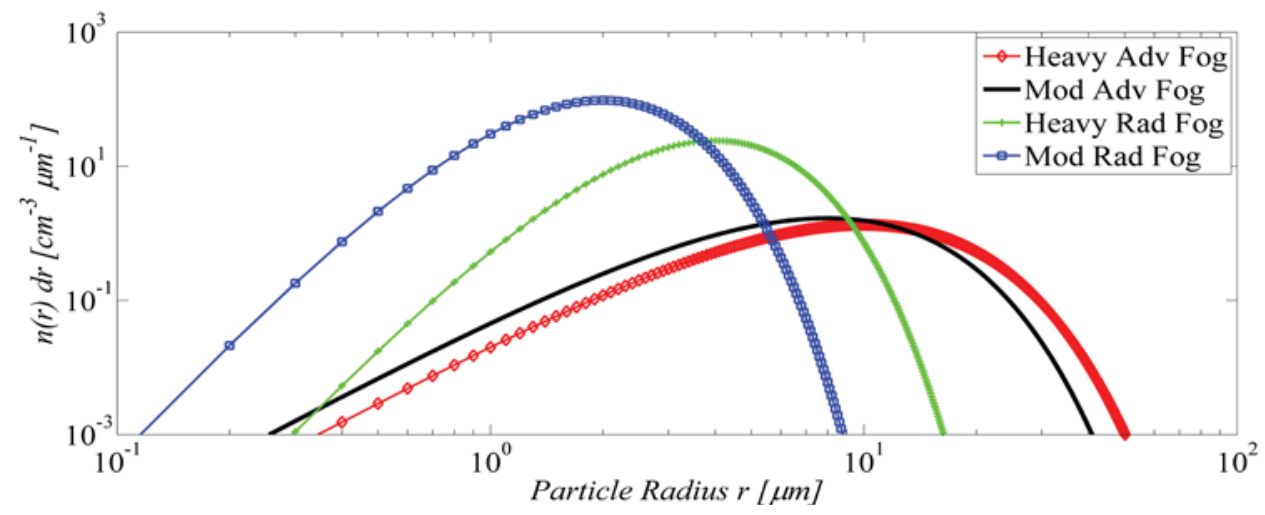

Figure 2.7 Modified Gamma size distribution function for fog aerosol models in accordance with data listed in table (2.2). 


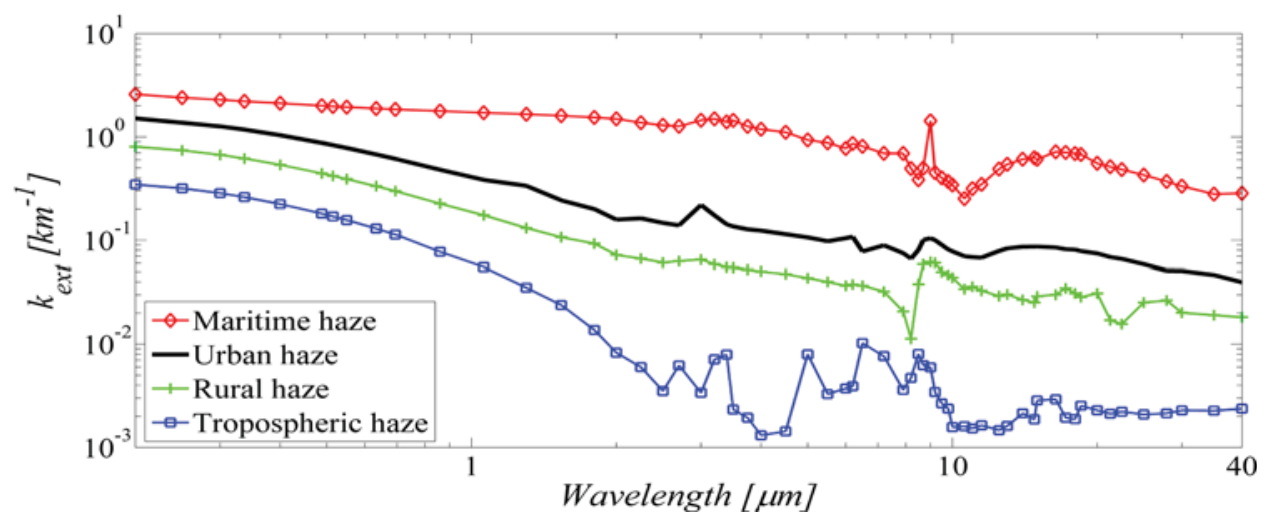

(a)

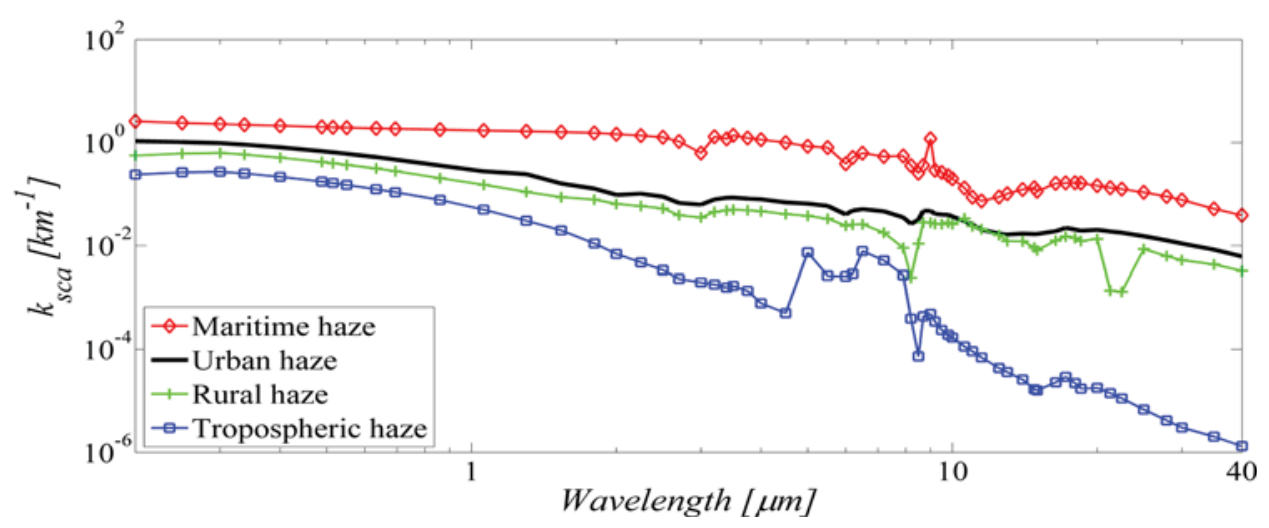

(b)

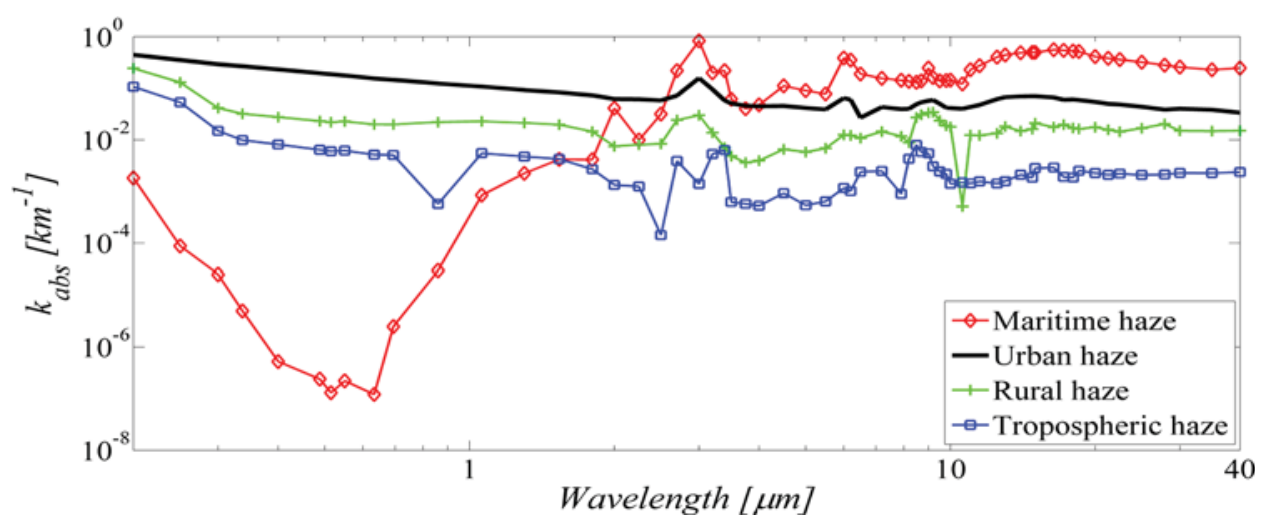

(c)

Figure 2.8 Optical properties of haze aerosol models in accordance with parameters listed in table (2.1).

(a) Extinction coefficient, $k_{\text {ext }}\left[\mathrm{km}^{-1}\right]$, (b) Scattering coefficient, $k_{s c a}\left[\mathrm{~km}^{-1}\right]$, (c) Absorption coefficient, $k_{a b s}\left[\mathrm{~km}^{-1}\right]$. 


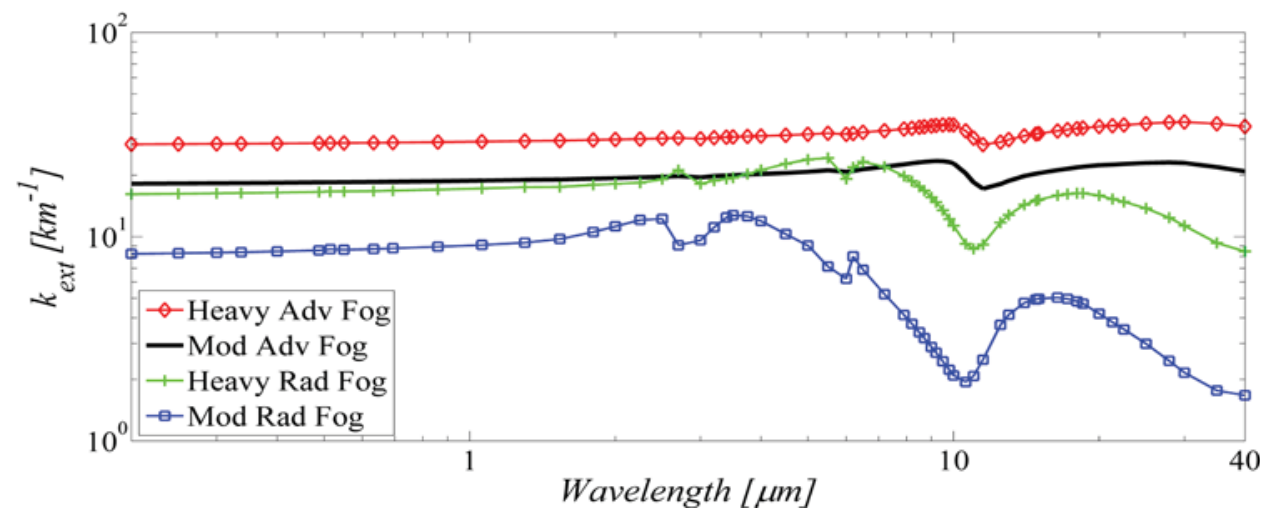

(a)

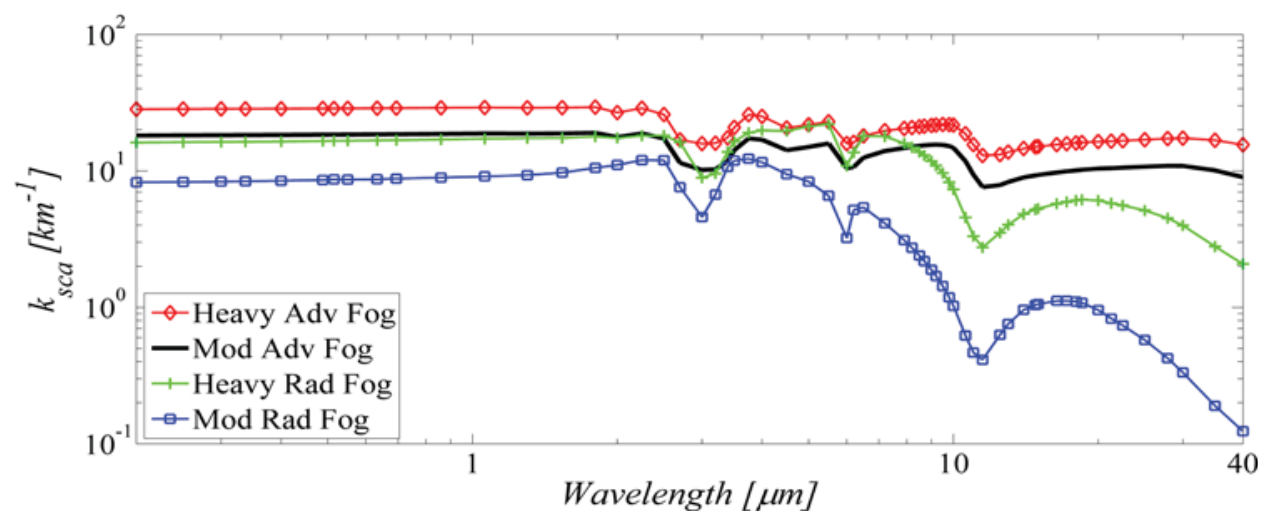

(b)

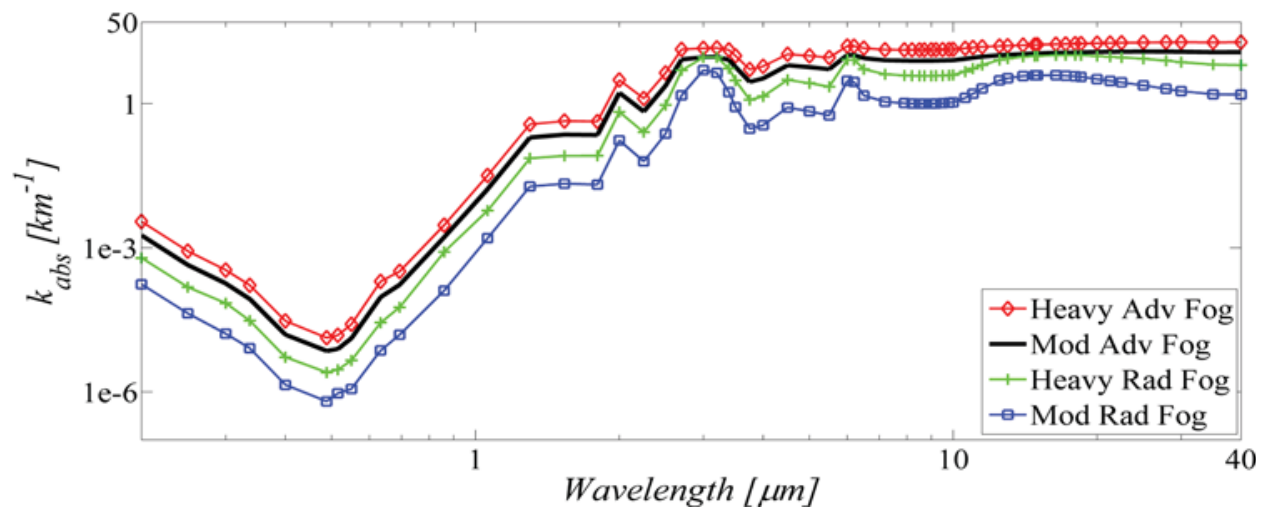

(c)

Figure 2.9 Optical properties of fog aerosol models in accordance with parameters listed in table (2.2).

(a) Extinction coefficient, $k_{\text {ext }}\left[\mathrm{km}^{-1}\right]$, (b) Scattering coefficient, $k_{s c a}\left[\mathrm{~km}^{-1}\right]$, (c) Absorption coefficient $k_{a b s}\left[\mathrm{~km}^{-1}\right]$. 


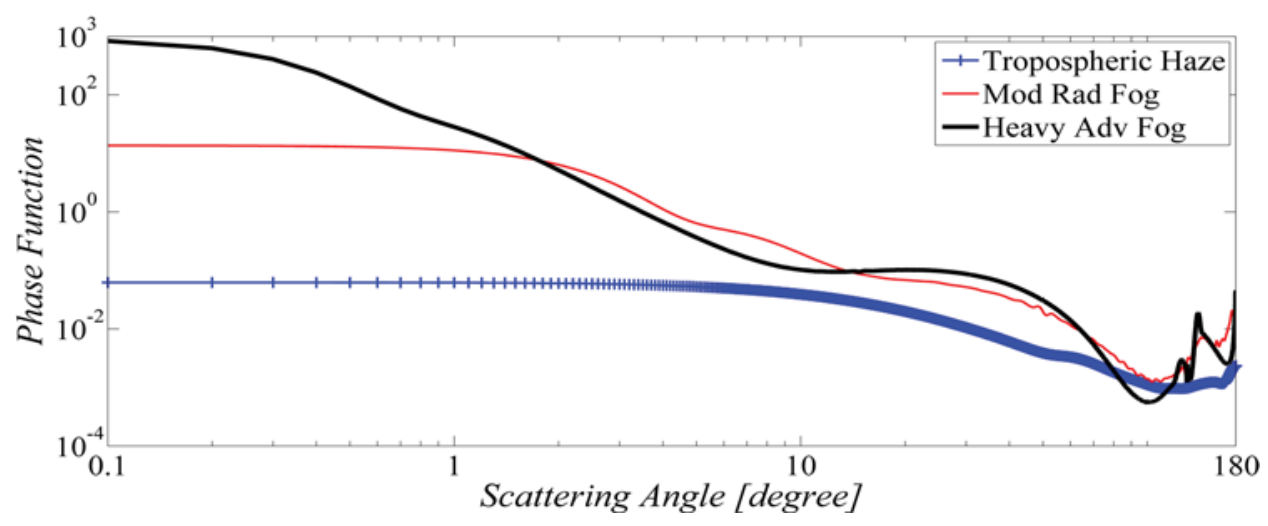

Figure 2.10 Phase function for tropospheric haze, moderate radiation fog, and heavy advection fog aerosol models.

\subsection{Model of the detected radiation}

The reduction in image quality is attributed either to the reduction of image contrast, due to atmospheric aerosols that cause extinction of the propagating radiation and scattering of such radiation at large enough angles leading to an ultimate reduction in the signal-tonoise ratio (SNR), or to image blurring, due to small angle scattering by atmospheric aerosols, where the scattered radiation collected by the sensor will be slightly deviated such that fine image features such as lines and edges are blurred. This spatial effect can be characterized as a broadening of the point spread function (PSF) [27]. Ben Dor et al. proposed a theoretical PSF model for different atmospheric haze and fog aerosol models, with various visibility values, in the visible and infrared spectral bands [6]. Bissonnette proposed another PSF model that discussed the effects of these atmospheric models on the PSF and compared the results with experimental data [20]. Both models confirm the addition of the direct PSF and scattered PSF at the image plane. The calculation of the average PSF of an imaging system accounts for the effects of the diffraction, scattering, 
and the appropriate optical power level of both the un-scattered (direct) and the scattered radiation arriving at the pupil of the imaging system. Since the scattered radiation is temporally and spatially de-correlated from the direct radiation, we model the effects of the direct (coherent) radiation and the scattered (incoherent) radiation of the aerosol species as additive in the image plane. It is worth mentioning that single scattering approach may be applied for atmospheric models with low optical depth $(\tau \leq 1)$, whereas multiple scattering approach is applied for optical depths of unity or more $(\tau>1)$. The following analysis accounts for values of $\tau>1$, and hence assumes a multiple scattering approach.

\subsubsection{Point spread function}

The PSF is the basic physical function that describes the spatial response of an imaging system. The angular distribution of the total detected PSF at the image plane, $P S F_{t}(\beta)$, consists of the sum of two independent components $[4,6,20]$,

$$
P S F_{\mathrm{t}}(\beta)=P S F_{d i r}(\beta)+P S F_{\text {sca }}(\beta)
$$

where $\beta$ is the angular distance in the image plane $[\mathrm{rad}], P S F_{\operatorname{dir}}(\beta)$ is the direct PSF, or the coherent intensity detected by the imaging system due to the direct radiation, and $P S F_{s c a}(\beta)$ is the scattered PSF, or the incoherent intensity due to the scattered radiation. Considering a plane wave propagating in an aerosol laden medium, with path length $z$, 
and an imaging system having focal length $f$ and a circular entrance aperture with diameter $D$. In the case of an atmospheric model with an AOD greater than $1 ; \tau>1$, $P S F_{d i r}(\beta)$ may be written as [4],

$$
P S F_{d i r}(\beta)=e^{-\tau}\left(\frac{D}{2 f \beta}\right)^{2} J_{1}^{2}\left(\frac{k D \beta}{2}\right)
$$

which is an attenuated Airy disc pattern. $P S F_{\text {sca }}(\beta)$ may be written as [4],

$$
P S F_{\text {sca }}(\beta)=\left(\frac{D}{2 \rho_{i}}\right)^{2} \exp \left[-\tau\left(1-\omega_{o}^{t}\right)-\left(\frac{f \beta}{\rho_{i}}\right)^{2}\right]
$$

where $\rho_{i}$ is the radial spread of the scattered PSF at the image plane $[\mathrm{m}]$, and is given by [4],

$$
\rho_{i}=0.26\left(\frac{\rho_{\text {Airy }} D}{\rho_{o}}\right)
$$

where $\rho_{\text {Airy }}=1.22 \lambda f / D$ is the Airy disk radius $[m]$, or the spatial spread of $P S F_{\operatorname{dir}}(\beta)$ at the image plane, $\rho_{o}$ is the correlation distance of the wave incident upon the entrance aperture $[m][4]$, 


$$
\rho_{o}=\frac{1.04 a_{\text {mode }}}{\sqrt{k_{s c a} z}}
$$

where $a_{\text {mode }}$ is the modal radius of the size distribution $[m]$. In many practical cases, $\rho_{o}$ is much smaller than the aperture size $D$, hence $P S F_{s c a}(\beta)$ is spread out at the image plane and the ratio of this spread, $\rho_{i}$, to the Airy disc radius, $\rho_{\text {Airy }}$, is proportional to the ratio of the entrance aperture size, $D$, to the correlation distance, $\rho_{o}[4]$. From Eqs. (2.19 - 2.23), a final form of the total detected PSF may be written as,

$$
\operatorname{PSF}_{t}(\beta)=\frac{D^{2}}{4}\left\{\left(\frac{e^{-\tau}}{f^{2} \beta^{2}}\right) J_{1}^{2}\left(\frac{k D \beta}{2}\right)+\left(\frac{1}{\rho_{i}^{2}}\right) \exp \left[-\tau\left(1-\omega_{o}^{t}\right)-\left(\frac{f \beta}{\rho_{i}}\right)^{2}\right]\right\}
$$

This formula is applied for each atmospheric aerosol species. If the medium comprises more than one aerosol species, then the detected PSF will be the weighted sum of the detected PSF from each species; i.e., $P S F_{\text {det }}(\beta)=\sum w_{i} P S F_{\operatorname{det}(i)}(\beta)$, with the weights $w_{i}$ given by the ratio of the number of aerosol of each species $\left(N_{i}\right)$ to the total number of aerosol found in the medium $(N), w_{i}=N_{i} / N$. A normalized total PSF is given by,

$$
P S F_{n}(\beta)=\frac{P S F_{d i r}(\beta)+P S F_{s c a}(\beta)}{P S F_{d i r}(0)+P S F_{s c a}(0)}
$$




\subsubsection{Case study}

The following case study investigates the effect of fog atmospheric aerosol models on the total PSF detected by an imaging system operating in the visible wavelength band. Assuming an atmospheric path length $z=500[\mathrm{~m}]$, the imaging system focal length $f=10$ $[\mathrm{cm}]$, and the entrance aperture diameter $D=4[\mathrm{~cm}]$. The intervening atmospheric models calculated optical parameters are listed in table (2.3). Figure (2.11) shows plots of the radial distribution of the detected PSFs at the image plane. Figure (2.11.a) shows the angular distribution of the direct PSF, $P S F_{\text {dir }}(\beta)$, figure (2.11.b) shows the radial distribution of the scattered PSF, $P S F_{s c a}(\beta)$, and figure (2.11.c) shows the radial distribution of the normalized total $\mathrm{PSF}, P S F_{n}(\beta)$. It is apparent that upon increased values of the AOD, $\tau$, due to the various fog aerosol models, the level of $P S F_{d i r}(\beta)$ decreases significantly, whereas the level of $P S F_{s c a}(\beta)$ increases. Also, $P S F_{\operatorname{dir}}(\beta)$ is narrowly concentrated in an Airy disc pattern, while $P S F_{s c a}(\beta)$ is widely spread throughout the entire image plane, due to the broad angle at which the scattered light

Table 2.3 Calculated optical parameters of fog atmospheric aerosol models for various meteorological range $(M)$ values.

\begin{tabular}{cccccc}
\hline \multirow{2}{*}{$\begin{array}{c}\text { Fog atmospheric aerosol } \\
\text { models }\end{array}$} & $M[\mathrm{~km}]$ & \multicolumn{4}{c}{ Calculated optical parameters } \\
\cline { 3 - 6 } & & $k_{\text {sca }}\left[\mathrm{km}^{-1}\right]$ & $\tau$ & $g$ & $\omega_{o}^{t}$ \\
\hline Heavy Advection & 0.136 & 28.7 & 14.37 & 0.872 & 1 \\
Moderate Advection & 0.212 & 18.8 & 9.38 & 0.869 & 1 \\
Heavy Radiation & 0.235 & 16.7 & 8.33 & 0.853 & 1 \\
Moderate Radiation & 0.453 & 8.6 & 4.32 & 0.831 & 1 \\
\hline
\end{tabular}




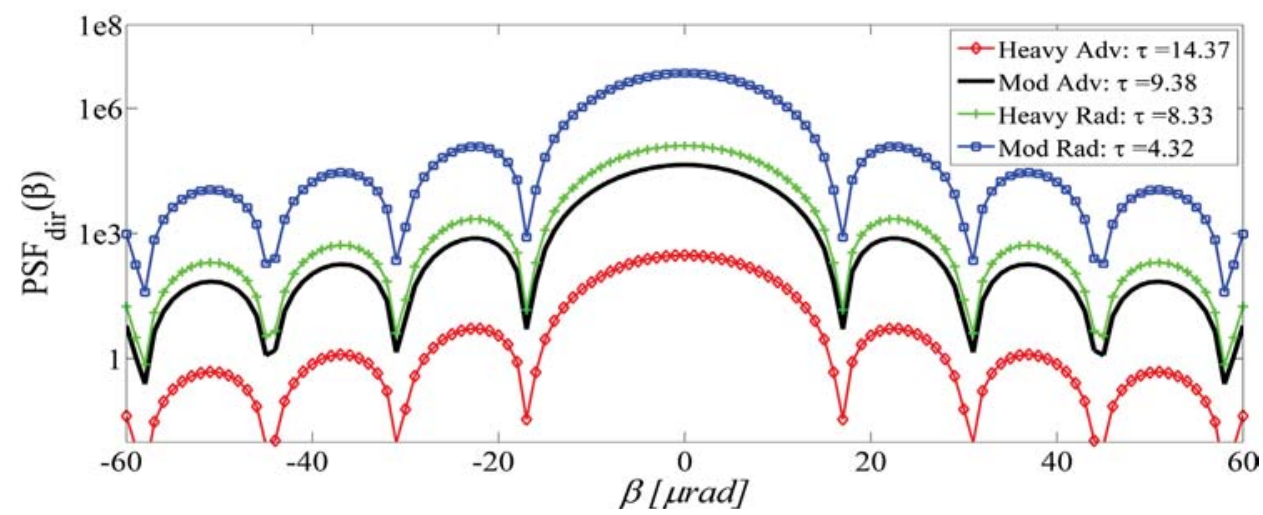

(a)

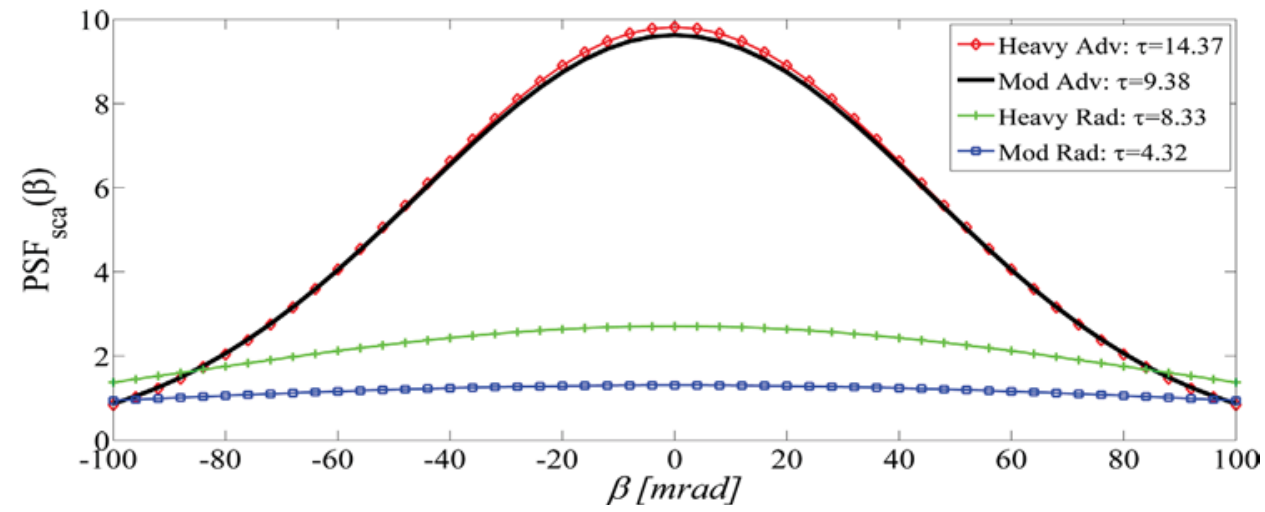

(b)

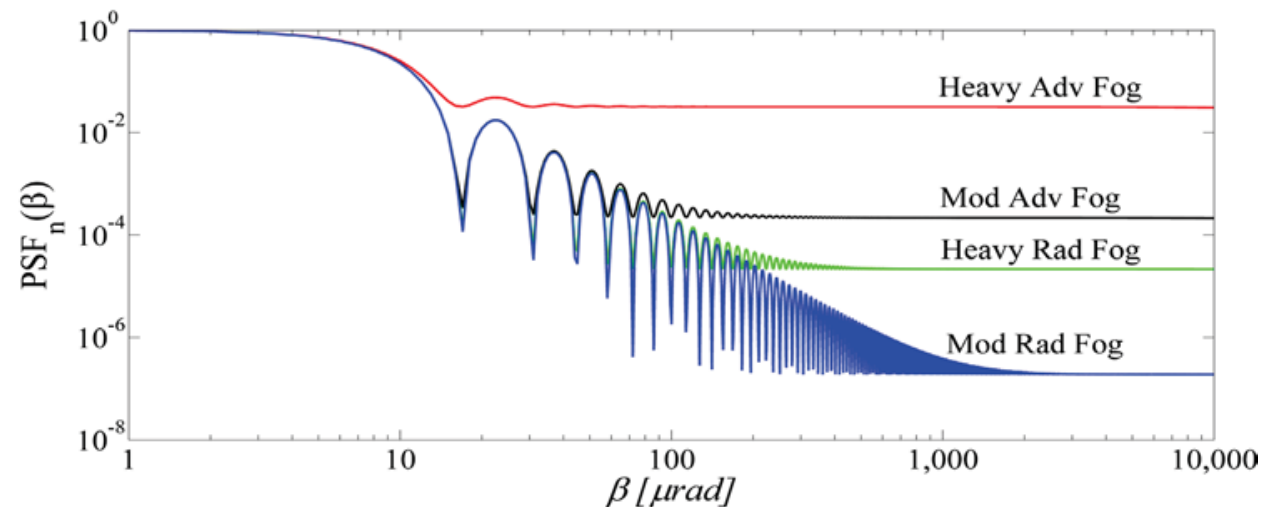

(c)

Figure 2.11 Angular distribution of the detected PSFs for fog aerosol models in accordance with the data listed in table (2.3).

(a) Direct (coherent) PSF, (b) Scattered (incoherent) PSF, (c) Normalized total PSF. 
arrives at the imaging system pupil, compared to the direct light. Hence, $P S F_{n}(\beta)$ is composed of two main components: a narrow central peak, representing the direct radiation from the source, and a weaker wide-spread wing, representing the scattered radiation by each of the fog models. An important conclusion is that in the central portion of the image plane, region near $\beta=0$, As long as $P S F_{d i r}>P S F_{\text {sca }}$, it could bepossible to get a reasonable image of a distant source. However, for an increasing $\tau$ values, as $P S F_{\text {sca }}(0)$ approaches $P S F_{d i r}(0)$ the image contrast decreases gradually until the image is completely unseen for $P S F_{\text {sca }}(0) \geq P S F_{\text {dir }}(0)$. The difference between this model and the model suggested by Ben Dor [6] is that this model is valid only for atmospheric aerosol models with an AOD greater than $1 ; \tau>1$, whereas Ben Dor's model is applied for very small values of the AOD in addition to $\tau>1$. Also, Ben Dor used a LOWTRAN7 code [28] to calculate the optical parameters of the atmosphere, however we calculated these parameters explicitly by the direct application of Mie scattering theory through our detailed EM model. This model agrees with Ben Dor's model in several aspects: the attenuation of the direct radiation and the increased level of the scattered radiation, detected by the imaging system, upon increased values of the AOD, the addition of the direct (coherent) and scattered (incoherent) PSFs at the imaging plane, and the significant effect of atmospheric aerosol on the image contrast. A very important observation can be inferred from figure (2.11.c) is that the angular distance, $\beta$, at which the normalized total PSF becomes approximately flat, is the angle corresponding to the reciprocal of the cutoff frequency of an aerosol MTF. Considering the imaging system focal length of 10 $[\mathrm{cm}]$ : For the heavy advection fog, moderate advection fog, heavy radiation fog, and moderate radiation fog, $\beta \approx 10,300,500,1000[\mu \mathrm{rad}]$, respectively, which is appropriate 
for an MTF cut-off frequencies of 100, 3.33, 2 and 1 [cycle/mrad]. Hence, for moderate radiation fog, the PSF is about 100 times broader than that of the heavy advection fog, and the MTF is about 100 times narrower. Aerosol MTF is thus for heavy advection fog a high spatial frequency phenomenon. This confirms the effect of increased level of the aerosols scattered radiation on limiting the spatial resolution of an imaging system.

\subsection{Conclusion}

This chapter deals with light scattering which is an important phenomenon that can significantly affect image quality through the atmosphere. The corner stone of developing any atmospheric model is determining and calculating the optical parameters of a single spherical particle using Mie scattering theory. This paper clarified the basic calculation of these parameters including the extinction coefficient, scattering coefficient, absorption coefficient, single scattering albedo, asymmetry parameter, and single scattering phase function. The combined effect of the particle size and composition, characterized by its complex refractive index, on these optical parameters is investigated. Also, characterizing the scattering and absorption of the propagating radiation through an atmospheric medium is mandatory to recognize the effect of the optical parameters of the overall medium on the quality of images acquired by an imaging system. The haze and fog atmospheric models are studied based on their particles size distribution functions and spectral refractive indices. We incorporated the optical parameters of various fog aerosol models into the calculation of the average point spread function due to the direct and 
scattered radiation acquired by an imaging system. The PSF model confirms the concentration of the coherent PSF, resulting from the acquired direct radiation, into an attenuated Airy pattern, whereas the incoherent PSF, that is due to the acquired scattered radiation, is spread throughout the image plane regarding to the fact that scattering has the effect of broadening the angle at which the scattered light arrives at the receiver compared to the direct light. Also, an increased atmospheric optical depth attenuates the level of the direct PSF and increases the level of the scattered PSF leading to a reduced image contrast. The level of the scattered radiation relative to the level of the direct radiation coming from a source determines the shape of the point spread function, where as long as $P S F_{d i r}>P S F_{s c a}$, it could be possible to get a reasonable image of a distant source. However, for an increasing atmospheric optical depth $\tau$, the image contrast decreases gradually until the image is completely unseen for $P S F_{s c a} \geq P S F_{\text {dir }}$. Finally, the analysis of fog aerosol models leads to the important conclusion of the limiting effect of aerosol scattering on the spatial resolution of the imaging system. 


\title{
Chapter 3
}

\section{Estimating The Image Spectrum Signal-}

\author{
to-Noise Ratio for Imaging Through
}

\section{Scattering Media}

2

2 The material contained in this chapter was submitted to SPIE Optical Engineering. See Appendix $\mathrm{C}$ for documentation of permission to republish this material. 


\subsection{Introduction}

The atmosphere limits the quality of images, particularly over long atmospheric paths. The main atmospheric distortions are attributed to atmospheric turbulence, and scattering and absorption by atmospheric aerosols $[\mathbf{1 , 1 4 - 1 6 , 2 7 ]}$. Light scattering by atmospheric aerosol media has the effect of light diffusion and broadening the angle at which the scattered light arrives at the receiver compared to the unscattered light, hence scattering decorrelates the light leaving the target from the unscattered light reaching the imaging system $[1,3]$. In imaging system design and performance prediction it is important to consider the detailed effects of atmospheric blur in order to minimize these effects where possible through careful optical design, and possibly to correct for atmospheric effects through post detection image reconstruction. For images measured through aerosol scattering media, the point spread function (PSF) is the basic physical function that describes the spatial response of an imaging system. The PSF of an imaging system is composed of two independent components; the direct PSF which is attributed to the direct, unscattered radiation arriving at the aperture of the imaging system, and the scattered PSF which is attributed to the scattered radiation due to the intervening atmosphere present between the target and the imaging system $[\mathbf{3 , 6}$. The modulus of the two dimensional Fourier transform of the PSF gives the modulation transfer function (MTF), which is a metric that describes how the spectral components of the target are affected by the propagation medium and the optical system [6, 29-32]. The PSF and MTF describe the limitations on image resolution imposed by the wave nature of light, the details of the imaging system design, and the propagation medium present between the 
target and the imaging system. However, it is also well-known that signal-to-noise ratio (SNR) considerations also affect the effective resolution of an imaging system [2]. In this paper we extend the approach of estimating the SNR of the spectral components of the Fourier transform of the measured image, referred to as the image spectrum SNR, described in [2] to the problem of understanding the noise-imposed limit on estimating the spectral components of the image. Analysis of the image spectrum SNR has been used to estimate the highest spatial frequency which can be measured with SNR of unity, referred to as the noise effective cutoff frequency. The reciprocal of the noise effective cutoff frequency defines the noise effective spatial resolution of an imaging system, which as a general rule is a less favorable estimate of resolution than obtained from wave optics considerations alone. The noise effective cutoff frequency also provides an estimate of the highest spatial frequency which can be reconstructed with a post detection image reconstruction algorithm [2]. In many cases of practical interest, the effects of scattering are quite strong, and limit the effective resolution of the imaging system. Previous work has addressed the effects of scattering on the overall PSF $[\mathbf{1 , 6 , 9 , 2 0 ]}$. Here we seek to extend these results to also account for the effects of reflected light, emitted light, and measurement noise, and to then estimate the noise effective resolution of the system. In this paper we use a previously published approach to estimating the effective PSF [1], and use radiometric calculations to estimate the mean numbers of direct and scattered photons due to both reflected and emitted light detected by an imaging system for a horizontal near-ground imaging scenario in the visible, NIR, and MIR spectral wavelength bands. 
The remainder of this paper is organized as follows. In Section 2 we describe the radiometric model for estimating the average number of photons arriving at the detector. The third section of the paper describes how the effective PSF is calculated as a function of the optical properties of haze and fog atmospheric aerosol models. In the fourth section, the PSF calculations are combined with the photon counting approach to identify the mean number of direct and scattered photons which are detected by the imaging system, which are then used to calculate the image spectrum SNR in the visible, NIR, and MIR spectral bands, for each of the atmospheric aerosol models. Conclusions are presented in the fifth section. Our key result is the demonstration of a technique for predicting the resolution limit of an imaging system working in the presence of fog and haze as a function of the optical system, the scattering medium, and the signal level.

\subsection{Image measurement model}

A radiometric technique for estimating the average number of photons detected by an imaging system is described in this section, for a horizontal near-ground imaging scenario. Figure (3.1) shows the geometry of the photon counting approach. Our analysis is concerned with the reflected solar radiation from an extended target in the visible and NIR spectral bands, and the thermal radiation that is emitted from the target in the MIR spectral band, as well as the scattering along the propagation path. The different parameters of the extended target, the imaging system, and the atmospheric medium that governs the radiative components used in estimating the average number of photons that 
are detected by the imaging system are included in figure (3.1). The extended target is assumed to be a Lambertian surface of area $A\left[\mathrm{~m}^{2}\right]$ and spectral reflectance $\rho(\lambda)$, the range between the target and the imaging system is $R[\mathrm{~m}]$, the diameter of the entrance aperture for the imaging system is $D[m]$, the imaging system focal length is $f[m]$, the optics transmittance is $\tau_{o p}$, the quantum efficiency of the detectors is $\eta$, and the integration time for the detectors is $t_{i n t}[s]$. The number of photons detected by an imaging system in the visible and NIR bands is calculated using the tabulated measurements of the extraterrestrial solar spectral irradiance $E^{o}(\lambda)\left[{W m^{-2}}^{\prime} m^{-1}\right]$, which is the observed spectral distribution of the solar irradiance at top of the atmosphere $[\mathbf{3 3}, \mathbf{3 4}]$; i.e. for a zero airmass; $m=0$. The air-mass, $m$, is defined as the path length which radiation takes through the atmosphere normalized to the shortest possible path length. The air-mass quantifies the reduction in the power of light, as it propagates through the atmosphere and is attenuated by atmospheric particles and aerosols, and is given as the secant of the solar zenith angle $z ; m=\sec (z)$, where $z$ is given by [34],

$$
z=\cos ^{-1}(\sin \theta \sin \delta+\cos \theta \cos \delta \cos \beta)
$$

where $\theta$ is the latitude of the target, $\delta$ is the solar declination of the day, and $\beta$ is the hour angle of the sun [34]. The values of $E^{o}(\lambda)$ used in this paper are derived from C. Wehrli extraterrestrial solar spectrum in the visible and NIR spectral wavelength band (0.39- 2 $\mu m)$ [33]. After propagation through the atmosphere, $E^{o}(\lambda)$ is attenuated, and the solar spectral irradiance at the target, $E(\lambda)\left[{W m^{-2}}^{\prime} m^{-1}\right]$, is given by Bouguer's law [34-36], 


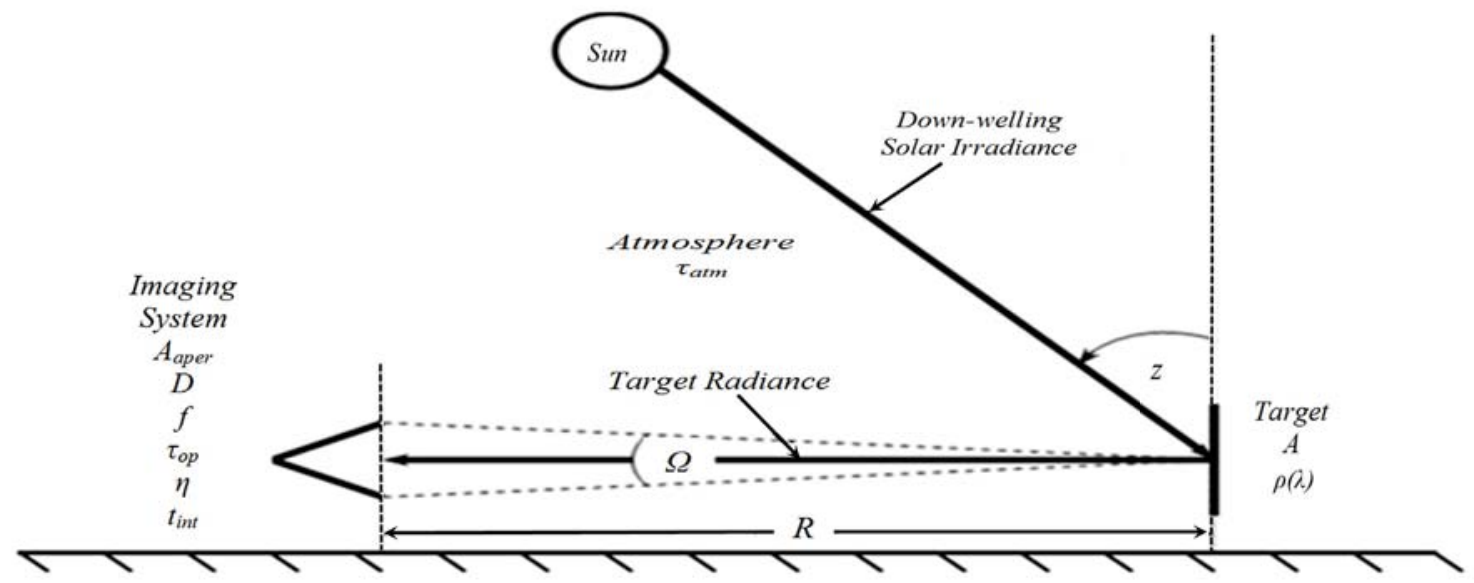

Figure 3.1 Geometry of the photon detection method.

$$
E_{\lambda}=E_{\lambda}^{o} \exp \left[-\tau_{\text {atm }}(\lambda) m\right]
$$

where $\tau_{\text {atm }}(\lambda)$ is the atmospheric optical depth, and is given by [35],

$$
\tau_{a t m}(\lambda)=\tau_{R}(\lambda)+\tau_{a}(\lambda)+\tau_{g}(\lambda)
$$

where $\tau_{R}(\lambda)$ is the Rayleigh optical depth, $\tau_{a}(\lambda)$ is the optical depth due to scattering by atmospheric aerosols, and $\tau_{g}(\lambda)$ is the optical depth due to molecular absorption by gases such as $\mathrm{O}_{3}, \mathrm{NO}_{2}$, and $\mathrm{H}_{2} \mathrm{O}$ [35]. Hence, the solar irradiance that illuminates the target depends on the optical properties of the intervening atmospheric medium, the target location, and the time within the day. The spectral exitance of the target $M(\lambda)\left[\mathrm{Wm}^{-2} \mu \mathrm{m}^{-1}\right]$ in the visible-NIR bands $(0.39-2 \mu m)$ is due to the down-welling solar spectral irradiance that has been reflected from the target; $M(\lambda)=\rho(\lambda) E(\lambda)$. Whereas, for the NIR-MIR 
spectral band $(2-5 \mu m), M(\lambda)$ is due to the thermal radiation emitted from the target and is assumed to be equivalent to the spectral exitance of a blackbody having a temperature of $300^{\circ} \mathrm{K}$ [37]. Figure (3.2) shows the calculated solar spectral irradiance at the target for different values of the air mass $m$, in the visible-NIR spectral wavelength bands, assuming an atmospheric medium without aerosols, $\tau_{a}(\lambda)=0$, and the thermal spectral exitance emitted from the target in the NIR-MIR bands. The spectral radiant flux intercepted by the imaging system, $\varphi(\lambda)\left[W \mu m^{-1}\right]$, can be calculated using the radiance theorem [37],

$$
\varphi(\lambda)=L(\lambda) A \Omega
$$

where $L\left[\mathrm{Wm}^{-2} s r^{-1} \mu m^{-1}\right]$ is the spectral radiance of the target; $L(\lambda)=M(\lambda) / \pi$, and $\Omega[s r]$ is the solid angle subtended by the entrance aperture of the imaging system at the target, as shown in Fig. 1; $\Omega=A_{\text {aper }} / R^{2}$, with $A_{\text {aper }}=\pi D^{2} / 4\left[\mathrm{~m}^{2}\right]$, is the area of the entrance aperture of the imaging system. The average number of photons detected at the detector plane of the imaging system in the small wavelength band $(\lambda, \lambda+\Delta \lambda), K_{\text {avg }}(\lambda)$, is given by [2],

$$
K_{a v g}(\lambda)=\frac{\Phi(\lambda) \tau_{a t m}(\lambda) \tau_{o p} \eta t_{\text {int }}}{E_{p h}(\lambda)}
$$




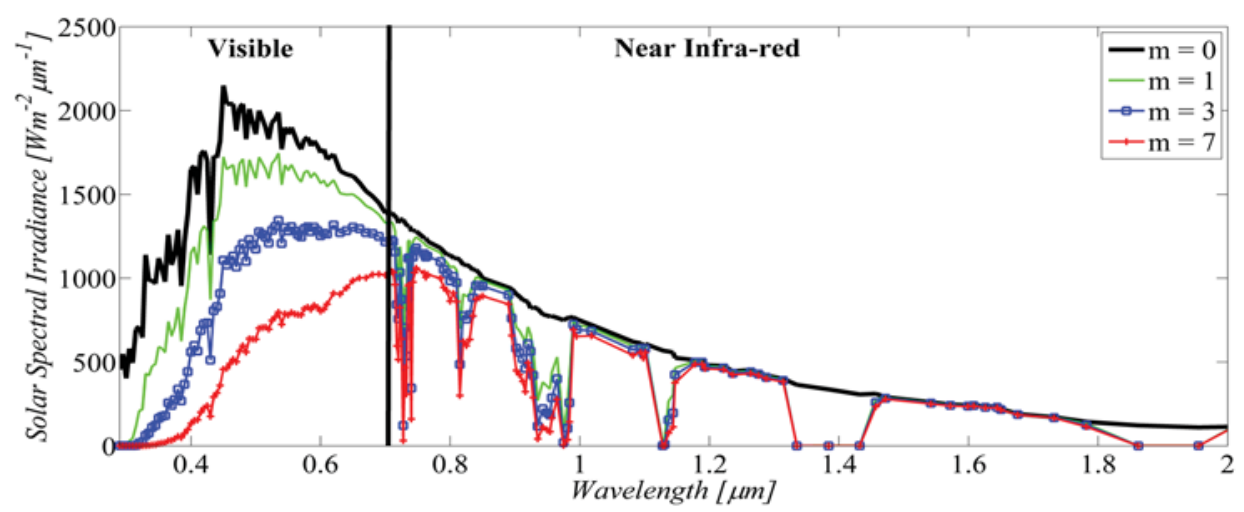

(a)

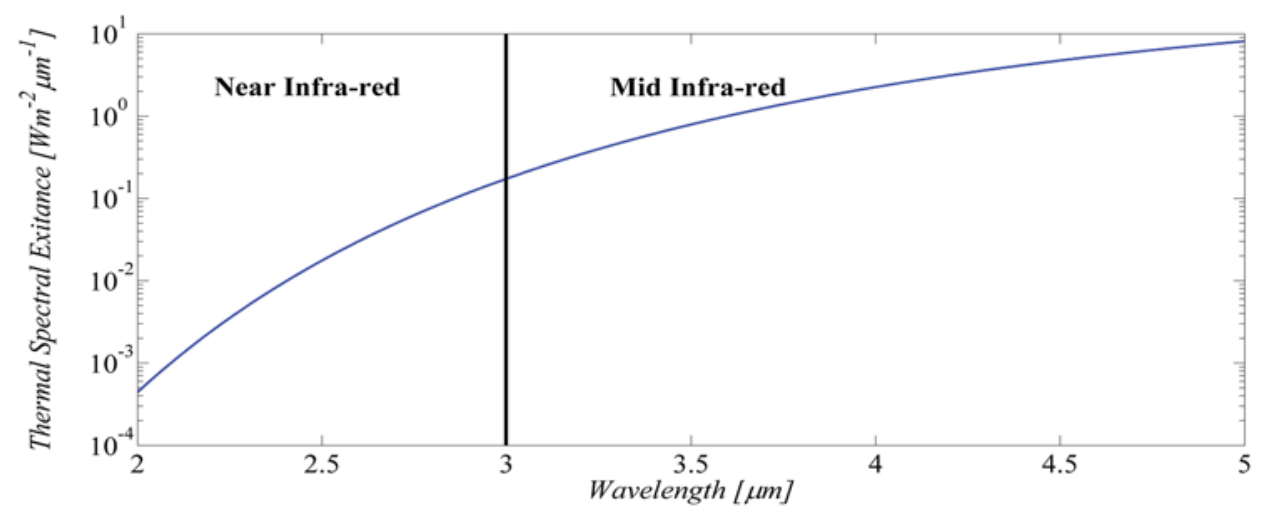

(b)

Figure 3.2 Solar spectral irradiance $\mathrm{E}(\lambda)$ at the visible-NIR bands, and thermal spectral exitance $\mathrm{M}(\lambda)$ at NIR-MIR bands for an extended target.

(a) Down-welling solar spectral irradiance at the target, $E(\lambda)$, for different values of the air mass $m$, in the visible and NIR spectral wavelength bands $(0.39-2 \mu m)$. (b) Thermal spectral exitance emitted from the target, $M(\lambda)$, at a temperature of $300^{\circ} \mathrm{K}$ in NIR-MIR bands $(2-5 \mu m)$.

where $\Phi(\lambda)[W]$ is the radiant flux in the wavelength band $(\lambda, \lambda+\Delta \lambda) ; \Phi(\lambda)=\varphi(\lambda) \Delta \lambda$ $[W]$, where we have used a value of $\Delta \lambda=5[\mathrm{~nm}]$ along the selected spectral wavelength intervals, $E_{p h}(\lambda)[J]$ is the photon energy, $E_{p h}(\lambda)=h c / \lambda$, where $h$ is Planck's constant, $h=$ $6.626 \times 10^{-34}[\mathrm{~J} . \mathrm{s}]$, and $c$ is the speed of light, $c=3 \times 10^{8}[\mathrm{~m} / \mathrm{s}]$. Hence, from Eqs. (3.4 - 
3.5), a final formula for calculating the average number of photons detected at the detector plane of the imaging system is given by,

$$
K_{\text {avg }}(\lambda)=\frac{A A_{\text {aper }} \tau_{\text {atm }}(\lambda) \tau_{\text {op }} \eta t_{\mathrm{int}} \lambda M^{\prime}(\lambda)}{\pi h c R^{2}}
$$

with $M^{\prime}(\lambda)\left[W^{-2}\right]$ is the radiant exitance of the target in the wavelength band $(\lambda, \lambda+\Delta \lambda)$; $M^{\prime}(\lambda)=M(\lambda) \Delta \lambda\left[\mathrm{Wm}^{-2}\right]$. This formula represents the average overall number of photons at a given wavelength, which include two categories; the first category represents the photons directly transmitted to the imaging system from the target, and the second category identifies the photons scattered by the atmospheric aerosols before reaching the imaging system. The proportions of the direct and scattered photons to the overall photons detected by the imaging system, as well as an estimation of the image spectrum SNR, will be discussed in the fourth section, based on the PSF calculations that will be presented in the third section.

\subsection{Effective point spread function}

Scattering and absorption by atmospheric aerosols varies greatly depending upon the aerosols composition, the aerosols size distribution function, the atmospheric conditions, and the region of the spectrum being examined $[3,38]$. In the visible-NIR spectral bands $(0.39-2.0 \mu \mathrm{m})$, scattering by atmospheric molecular gases and aerosols is the dominant 
contributor to atmospheric path radiance [39]. This motivates us to investigate the contribution of the effective PSF of the imaging system to the average number of photons reaching the sensor plane of the imaging system in the visible, NIR, and MIR spectral wavelength bands, for different haze and fog aerosol models, according to the optical properties of these atmospheric models. Three haze aerosol models and three fog aerosol models have been selected for the investigation. The haze models include urban, rural, and tropospheric haze atmospheric aerosol models, whereas the fog models include moderate radiation, heavy radiation, and heavy advection fog aerosol models. The optical properties of the atmospheric aerosol models, including their extinction coefficient, $k_{\text {ext }}(\lambda)$ $\left[m^{-1}\right]$, scattering coefficient, $k_{s c a}(\lambda)\left[m^{-1}\right]$, absorption coefficient, $k_{a b s}(\lambda)\left[m^{-1}\right]$, scattering albedo, $\omega_{o}(\lambda)$, asymmetry parameter, $g(\lambda)$, and the modal radius, $a_{\text {mode }}[\mu m]$, of their size distribution functions, for different values of the meteorological range $M[m]$, along the visible, NIR and MIR spectral wavelength bands, are listed in Table (3.1). Also, plots of $k_{\text {ext }}(\lambda), k_{\text {sca }}(\lambda)$ and $k_{a b s}(\lambda)$ for the haze and fog aerosol models are shown in figure (3.3). The radial distribution of the effective PSF in the imaging plane is composed of two independent components, direct PSF, $P S F_{\operatorname{dir}}(r, \lambda)$, or the coherent intensity detected by the imaging system due to the direct radiation, and scattered PSF, $P S F_{s c a}(r, \lambda)$, or the incoherent intensity due to the scattered radiation. $P S F_{d i r}(r, \lambda)$ is given by an attenuated diffraction-limited Airy disc pattern [4],

$$
P S F_{d i r}(r, \lambda)=\exp \left[-\tau_{\text {atm }}(\lambda)\right]\left(\frac{D}{2 r}\right)^{2} J_{1}^{2}\left(\frac{k D r}{2 f}\right)
$$

where $r[m]$ is the radial distance in the imaging plane, $k\left[\mathrm{~m}^{-1}\right]$ is the wave number of the 
Table 3.1 Optical properties of haze and fog atmospheric aerosol models in the visible, NIR, and MIR spectral wavelength bands $(0.39-5 \mu \mathrm{m})$.

\begin{tabular}{|c|c|c|c|c|c|c|c|}
\hline \multirow{2}{*}{$\begin{array}{c}\text { Atmospheric } \\
\text { aerosol } \\
\text { models }\end{array}$} & \multirow[b]{2}{*}{$M[\mathrm{~km}]$} & \multicolumn{6}{|c|}{ Calculated optical properties $(\lambda=0.39-5 \mu \mathrm{m})$} \\
\hline & & $\begin{array}{l}a_{\text {mode }} \\
{[\mu \mathrm{m}]}\end{array}$ & $\omega_{o}^{t}$ & $g(\lambda)$ & $\begin{array}{l}k_{\text {ext }}(\lambda) \\
{\left[\mathrm{km}^{-1}\right]}\end{array}$ & $\begin{array}{l}k_{\text {sca }}(\lambda) \\
{\left[\mathrm{km}^{-1}\right]}\end{array}$ & $\begin{array}{l}k_{a b s}(\lambda) \\
{\left[\mathrm{km}^{-1}\right]}\end{array}$ \\
\hline $\begin{array}{l}\text { Tropospheric } \\
\text { haze }\end{array}$ & 25 & 0.021 & $0.95-0.91$ & $0.66-0.39$ & $\begin{array}{c}0.31- \\
0.8 \times 10^{-3}\end{array}$ & $\begin{array}{c}0.29- \\
0.7 \times 10^{-3}\end{array}$ & $\begin{array}{c}0.016- \\
0.68 \times 10^{-4}\end{array}$ \\
\hline Rural haze & 10 & 0.011 & $0.94-0.87$ & $0.68-0.64$ & $0.72-0.04$ & $0.68-0.04$ & $\begin{array}{c}0.04- \\
0.57 \times 10^{-3}\end{array}$ \\
\hline Urban haze & 5 & 0.012 & $0.77-0.64$ & $0.77-0.65$ & $1.30-0.11$ & $1-0.07$ & $0.30-0.04$ \\
\hline $\begin{array}{l}\text { Moderate } \\
\text { radiation fog }\end{array}$ & 0.453 & 2 & 0.97 & $0.84-0.72$ & $8.37-9.03$ & $8.37-10.98$ & $\begin{array}{c}1.7 \times 10^{-5}- \\
8.1 \times 10^{-4}\end{array}$ \\
\hline $\begin{array}{l}\text { Heavy } \\
\text { radiation fog }\end{array}$ & 0.235 & 4 & 0.98 & $0.86-0.74$ & $16.29-23.87$ & $16.29-18.08$ & $\begin{array}{c}6.1 \times 10-5- \\
3.3 \times 10^{-3}\end{array}$ \\
\hline $\begin{array}{c}\text { Heavy } \\
\text { advection fog }\end{array}$ & 0.136 & 10 & 0.99 & $0.87-0.83$ & $28.45-31.7$ & $28.44-29.85$ & $\begin{array}{c}3.5 \times 10^{-4}- \\
1.7 \times 10^{-2}\end{array}$ \\
\hline
\end{tabular}

propagating radiation, $k=2 \pi / \lambda$, and $J_{1}$ is Bessel function of the first kind of order 1 . $\operatorname{PSF}_{\text {sca }}(r, \lambda)$ is given by $[\mathbf{3}, \mathbf{4}]$,

$$
P S F_{s c a}(r, \lambda)=\left(\frac{1.64 a_{\text {mod } e} D}{\lambda f \sqrt{R k_{s c a}(\lambda)}}\right) \exp \left\{-\tau_{\text {atm }}(\lambda)\left[1-\omega_{o}^{t}(\lambda)\right]-\left(\frac{3.28 a_{\text {mode }} r}{\lambda f \sqrt{R k_{s c a}(\lambda)}}\right)\right\}
$$

In order to restrict our analysis to the effect of varying the atmospheric optical depth, $\tau_{\text {atm }}(\lambda)$, on the PSF due to the scattering atmospheric aerosol models, and consequently on the photon count, all the parameters shown in figure (3.1) for the imaging system and the target will be kept constant, except the range, $R$, between the target and the imaging system. The criterion that will be used to estimate the mean numbers of direct and scattered portions of photons that reach the imaging system depends on calculating 


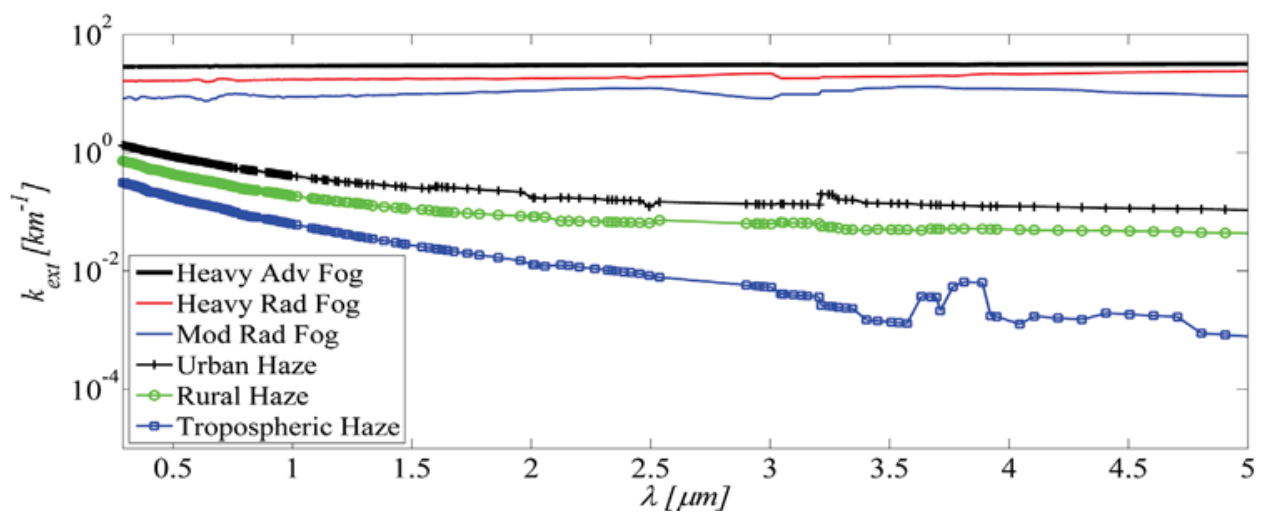

(a)

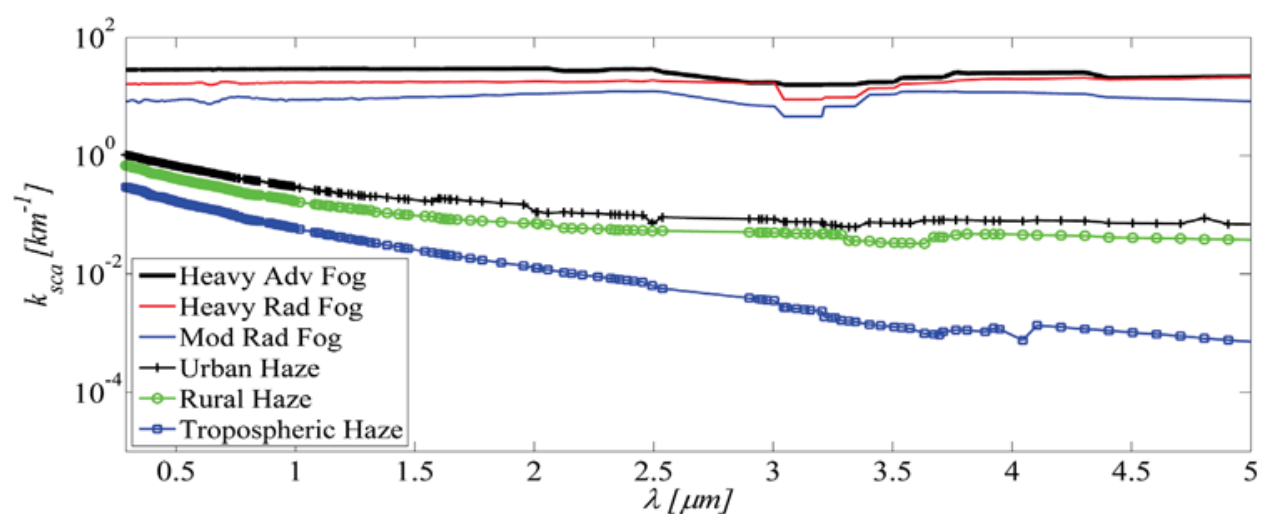

(b)

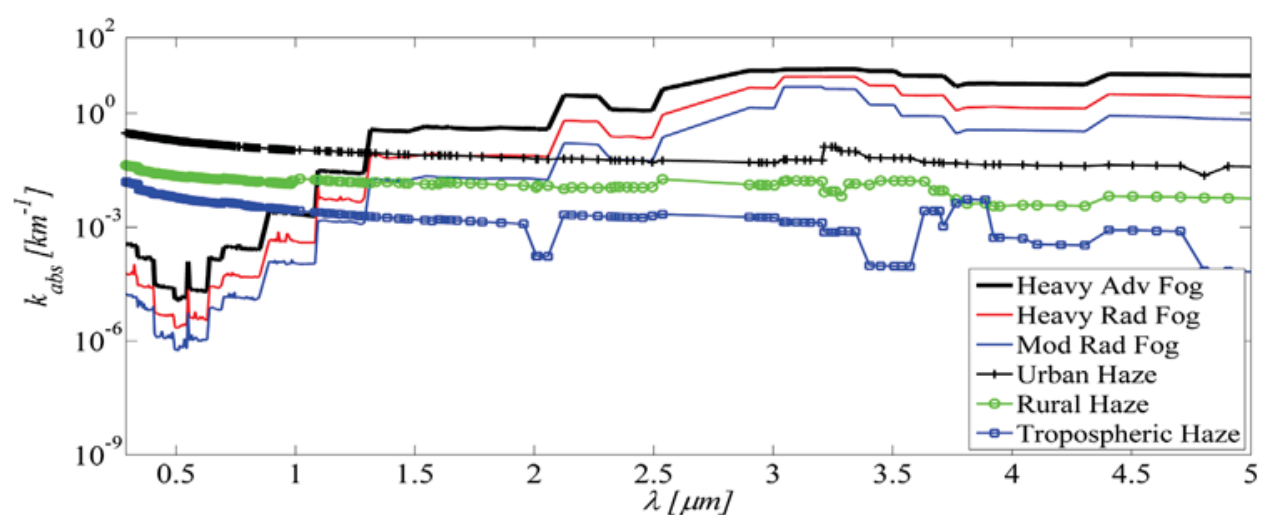

(c)

Figure 3.3 Optical properties of the haze and fog atmospheric aerosol models.

(a) Extinction coefficient $k_{\text {ext }}\left[\mathrm{km}^{-1}\right]$. (b) Scattering coefficient $k_{s c a}\left[\mathrm{~km}^{-1}\right]$. (c) Absorption coefficient $k_{a b s}\left[\mathrm{~km}^{-1}\right]$. 
$P S F_{d i r}(r, \lambda)$ and $P S F_{s c a}(r, \lambda)$ at the central portion of the imaging plane, since $\operatorname{PS} F_{\operatorname{dir}}(r, \lambda)$ and $P S F_{s c a}(r, \lambda)$ achieve their maximum values at this portion. Figure (3.4) shows $P S F_{d i r}(0, \lambda)$ and $P S F_{s c a}(0, \lambda)$ versus wavelength, $\lambda$, for haze and fog aerosol models, along different values of $\tau_{a t m}$, and in accordance with the optical properties of the atmospheric models listed in Table (3.1). It is noticed from figure (3.4) that the level of $P S F_{\text {sca }}$ approaches $P S F_{d i r}$ along the entire spectral wavelength band upon increased values of the optical depth, $\tau_{a t m}$, for each atmospheric aerosol model. This approach is more apparent for the fog aerosol models, figure (3.4-b), than that for the haze aerosol models, figure (3.4-a), due to the presence of a stronger forward-scattering by the large-sized fog aerosols than the weak scattering encountered by the small-sized haze aerosols. The impact of increasing the atmospheric optical depth, due to various aerosol models, on the levels of the direct and scattered PSFs will be used to calculate the mean numbers of direct and scattered photons that will be used to estimate the image spectrum SNR, as will be clarified in the next section.

\subsection{Image spectrum signal-to-noise ratio calculations}

In this section we first describe how the contributions of the direct and scattered components of the effective PSF are used to calculate the mean numbers of direct and scattered photons, and then show how to calculate the image spectrum SNR. 


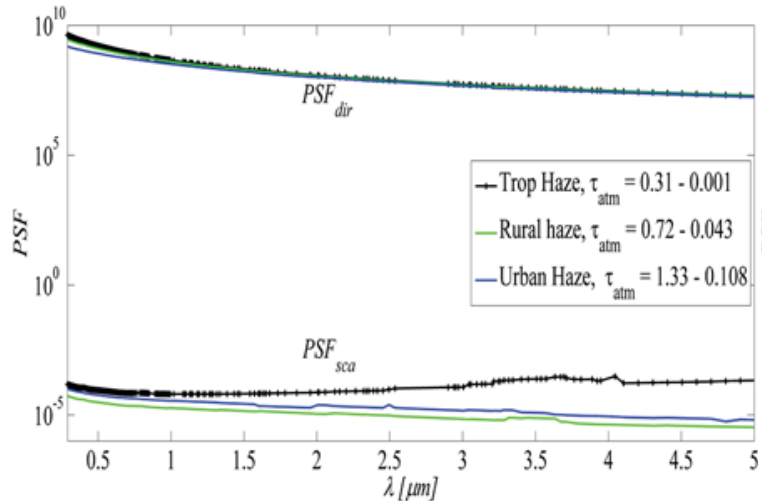

(i) $R=1000[\mathrm{~m}]$

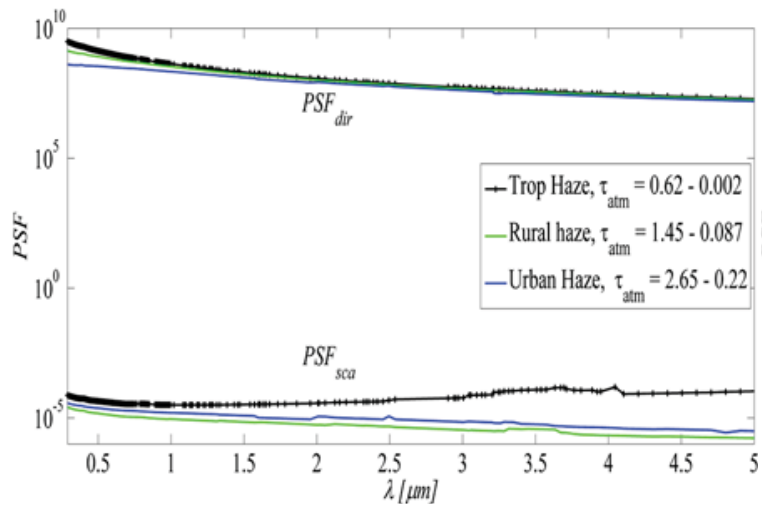

(ii) $R=2000[\mathrm{~m}]$

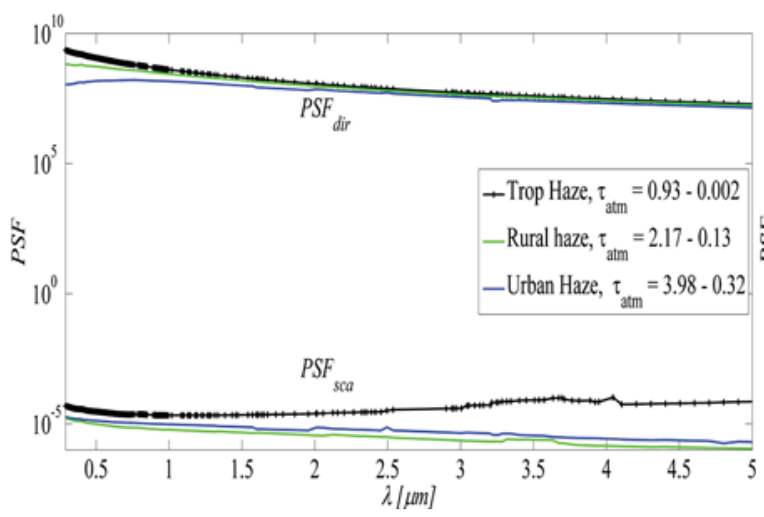

(iii) $R=3000[\mathrm{~m}]$

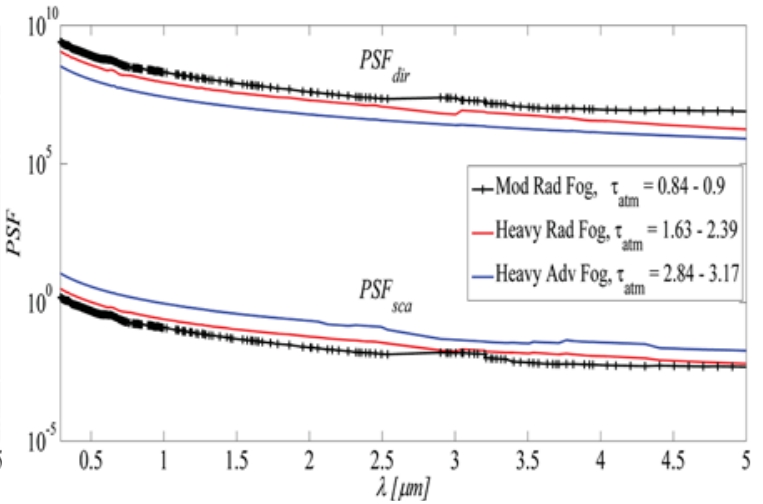

(i) $R=100[\mathrm{~m}]$

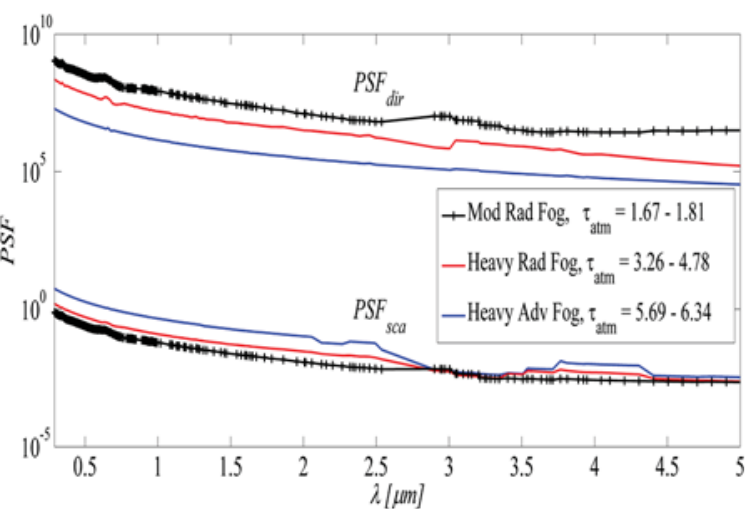

(ii) $R=200[\mathrm{~m}]$

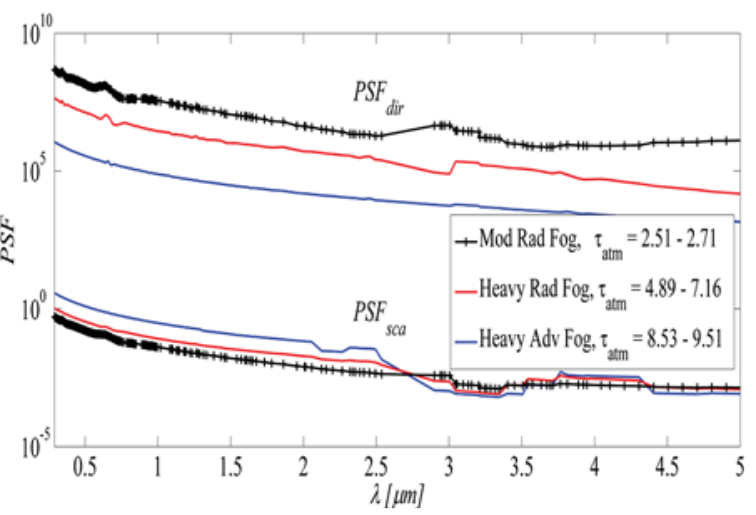

(iii) $R=300[\mathrm{~m}]$

(a)

(b)

Figure 3.4 $P \operatorname{PF}_{\operatorname{dir}}(0, \lambda)$ and $P S F_{s c a}(0, \lambda)$ for atmospheric aerosol models along different values of range $R$.

(a) Haze aerosol models, (b) Fog aerosol models 


\subsubsection{Calculation of the numbers of direct and scattered photons}

Eqs. (3.6 - 3.8) may be used to calculate the mean numbers of direct photons, $K_{d i r}(\lambda)$, and scattered photons, $K_{\text {sca }}(\lambda)$, reaching the detector of the imaging system as,

$$
\begin{gathered}
K_{\text {dir }}(\lambda)=\left[\frac{P S F_{\text {dir }}(0, \lambda)}{P S F_{t}(0, \lambda)}\right] K_{\text {avg }}(\lambda) \\
K_{\text {sca }}(\lambda)=\left[\frac{P S F_{\text {sca }}(0, \lambda)}{P S F_{t}(0, \lambda)}\right] K_{\text {avg }}(\lambda)
\end{gathered}
$$

Where $\operatorname{PSF}_{t}(0, \lambda)$ is the total effective PSF at the central portion of the imaging plane, and is given as the addition of the direct and scattered PSFs; $\operatorname{PSF}_{t}(0, \lambda)=\operatorname{PSF}_{\operatorname{dir}}(0, \lambda)+$ $P S F_{\text {sca }}(0, \lambda)[\mathbf{1 , 6 , 1 5 , 3 9 ]}$. Considering a photon-limited imaging system having $D=0.28$ $[\mathrm{m}], f=2.8[\mathrm{~m}], \tau_{o p}=1, \eta=1$, and $t_{\text {int }}=15 \times 10^{-3}[\mathrm{~s}]$, and assuming an ideal Lambertian target, and an air mass, $m=1.5$. According to Eqs. $(3.7-3.10)$ and table (3.1), the average number of direct and scattered photons for the haze and fog aerosol models are shown in figure (3.5). It is noted that no photons are recorded within the opaque atmospheric windows $1.3-1.44[\mu \mathrm{m}], 1.78-2[\mu \mathrm{m}]$, and $2.45-2.9[\mu \mathrm{m}]$, due to the reduced radiation transmission through these spectral bands. Figure (3.5.b) shows the increased proportion of the number of scattered photons, relative to the number of direct photons, upon increased atmospheric depth, specially for the heavy advection fog. 


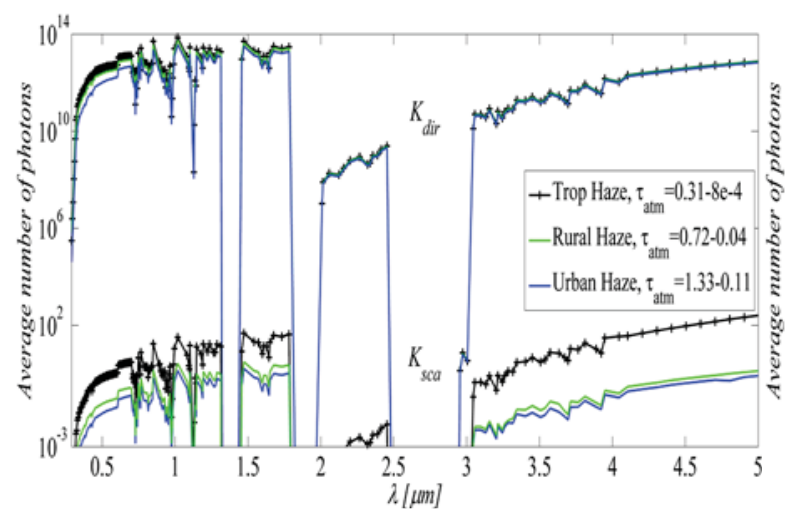

(i) $R=1000[\mathrm{~m}]$

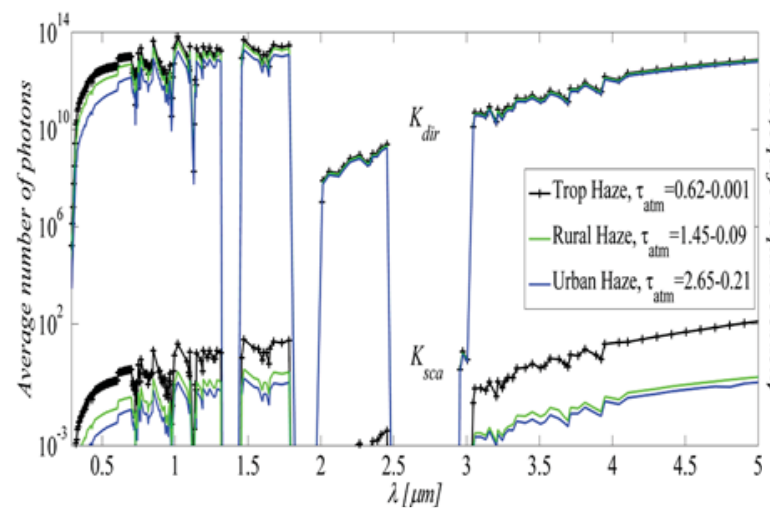

(ii) $R=2000[\mathrm{~m}]$

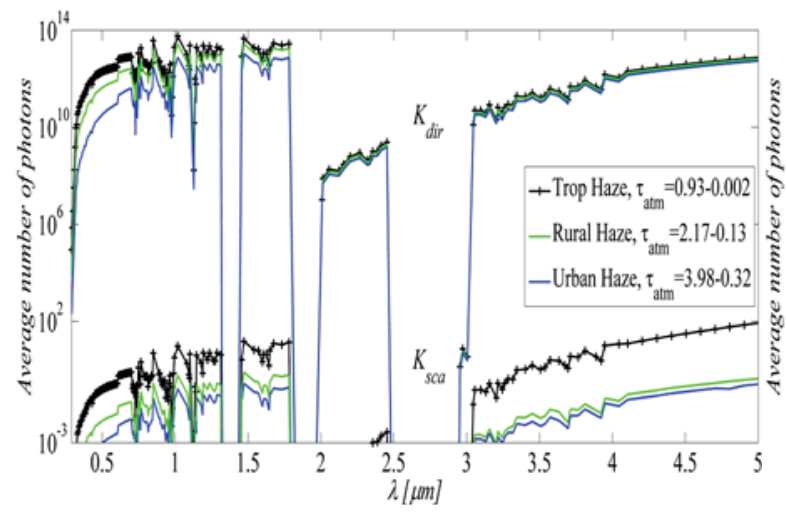

(iii) $R=3000[\mathrm{~m}]$

(a)

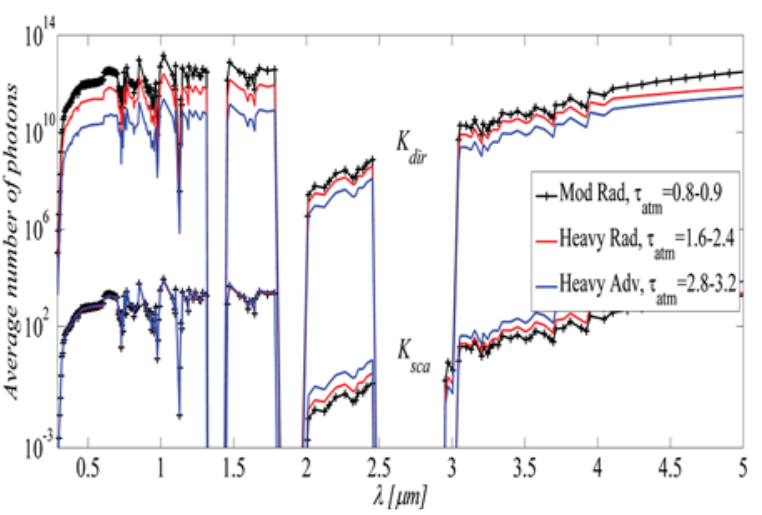

(i) $R=100[\mathrm{~m}]$

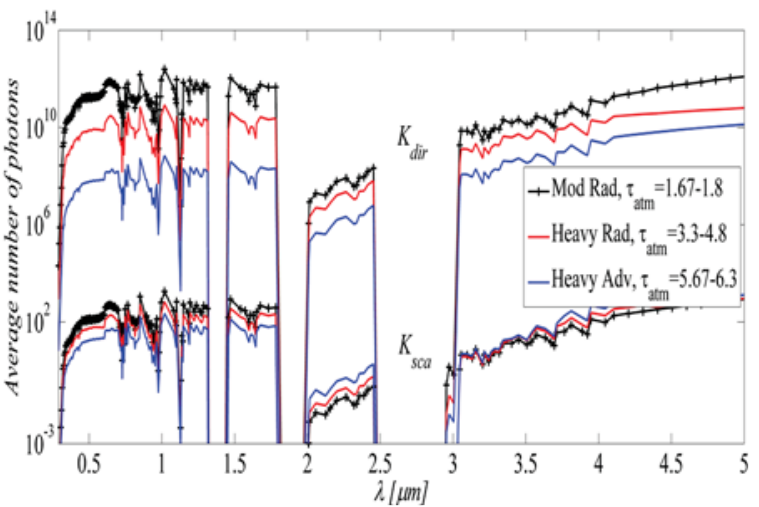

(ii) $R=200[\mathrm{~m}]$

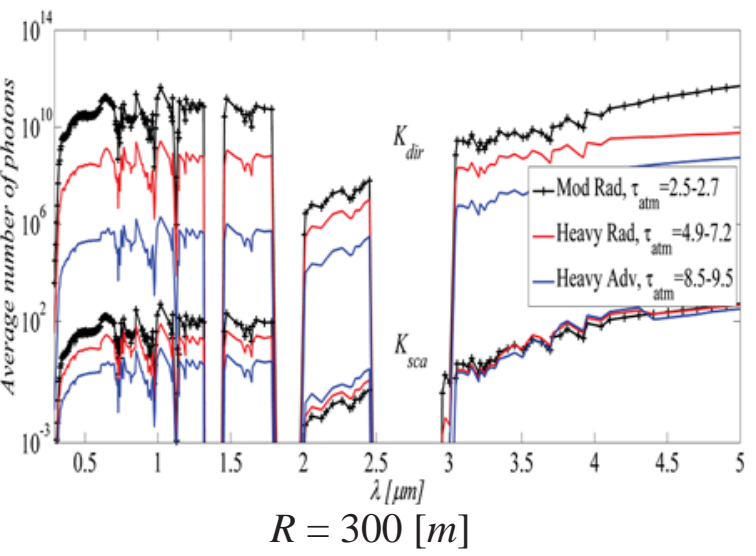

(b)

Figure 3.5 Average number of direct and scattered photons, $K_{d i r}$ and $K_{s c a}$, for atmospheric aerosol models along different values of range $R$.

(a) Haze aerosol models, (b) Fog aerosol models. 


\subsubsection{Calculation of the image spectrum SNR}

The mean number of direct and scattered photons, are now used to calculate the image spectrum SNR of a detected image at the visible, NIR, and MIR spectral bands. Following the development in [2], let $I(v)$ be the Fourier transform of an image which has been detected with a photon counting camera, where $v$ is the spatial frequency [cycle/rad]. Image spectrum SNR is defined as the ratio of the mean of $I(v)$ to the standard deviation of $I(v)$. The image spectrum SNR is a measure of the relative strengths of the image spectrum signal and the random variability of the signal in the spatial frequency domain, hence providing a useful metric of evaluating the effects of various sources of noise on the image quality [2]. The image SNR provides a means of estimating the noise effective resolution of an imaging system, and a means of estimating the highest frequency which can be reconstructed with a post detection image reconstruction algorithm, The SNR of an image obtained with a deterministic MTF and a photon-limited image detection system is given by [2],

$$
\operatorname{SNR}(v)=\frac{K_{d i r}\left|H(v) O_{n}(v)\right|}{\sqrt{K_{d i r}+K_{s c a}}}
$$

where $\operatorname{SNR}(v)$ is the image spectrum signal-to-noise ratio, $H(v)$ is the MTF of the imaging system, and $O_{n}(v)$ is the normalized Fourier spectrum of the target irradiance distribution. The cutoff spatial frequency, $v_{c u t}[\mathrm{cycle} / \mathrm{rad}]$, of an imaging system operating in a spectral wavelength band, is given by [40], 


$$
v_{c u t}=\frac{D}{\lambda_{c}}
$$

where $\lambda_{c}[m]$ is the central wavelength of the spectral band. Also, another parameter to be defined is the noise effective cutoff spatial frequency, vnoise [cycle/rad], of the image spectrum SNR, which is the spatial frequency that corresponds to an image spectrum SNR value of 1 . The reciprocal of $v_{\text {noise }}$ indicates a threshold noise effective spatial resolution, $\theta_{t h}[\mathrm{rad}]$, under which the noise exceeds the signal in a given image; i.e., if the angular spatial resolution gets smaller than $\theta_{t h}$, the noise recorded in the image due to scattering will exceed the signal level. Figure (3.6) shows the radially-averaged image spectrum SNR, $S N R_{r a d}$, versus the spatial frequency, $v$, for the haze and fog aerosol models at the visible; $\lambda_{c}=0.55[\mu m]$, NIR; $\lambda_{c}=1.22[\mu m]$, and MIR; $\lambda_{c}=4[\mu m]$, spectral wavelength bands, at $R=3000[\mathrm{~m}]$ for the haze aerosol models, and at $R=300[\mathrm{~m}]$ for the fog aerosol models. The values of $K_{d i r}$ and $K_{s c a}$ are extracted from figure (3.4-a-iii) and figure (3.4-b-iii) at the specified central wavelengths for each spectral band, and are used in Eq. (3.11) to estimate the SNR for each spectral band. The diffraction-limited plots, shown in figure (3.6), assume the absence of atmospheric aerosols; $K_{s c a}=0$. Table 2 lists the estimated noise effective cutoff spatial frequency, $v_{\text {noise }}$ [cycle/rad], of the image spectrum SNR for the atmospheric aerosol models, for each spectral band as shown in figure (3.6), and the corresponding threshold noise effective spatial resolution, $\theta_{t h}[\mathrm{rad}]$. Figure (3.6) and table (3.2) indicate that for each atmospheric aerosol model the 

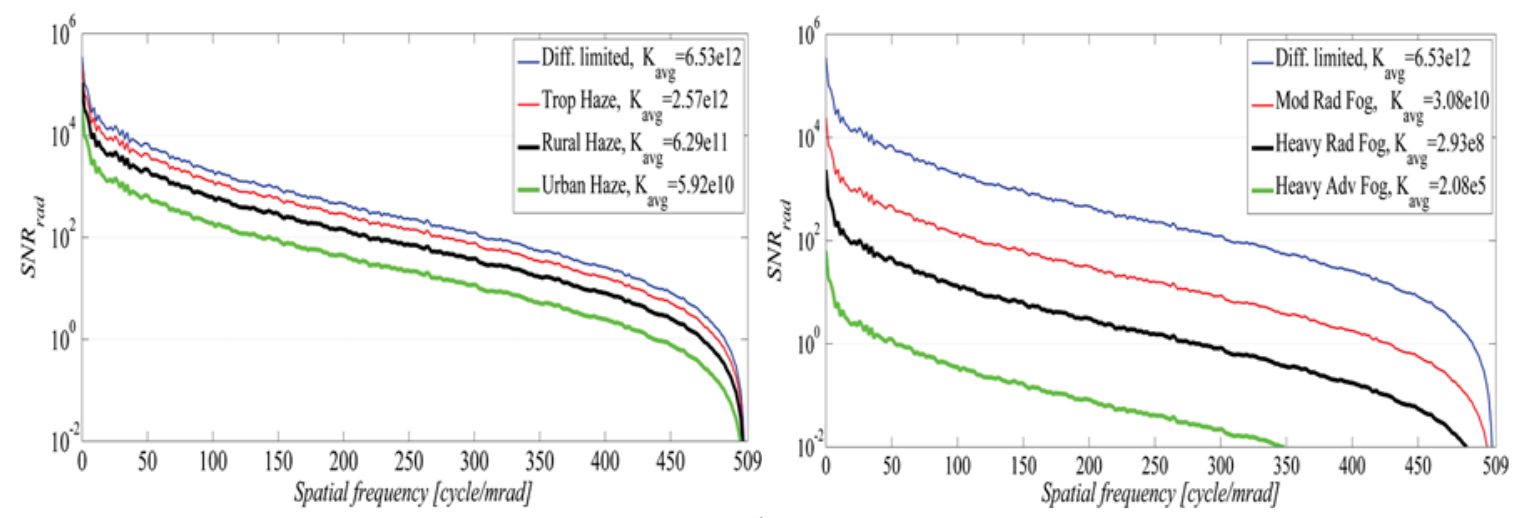

(i)
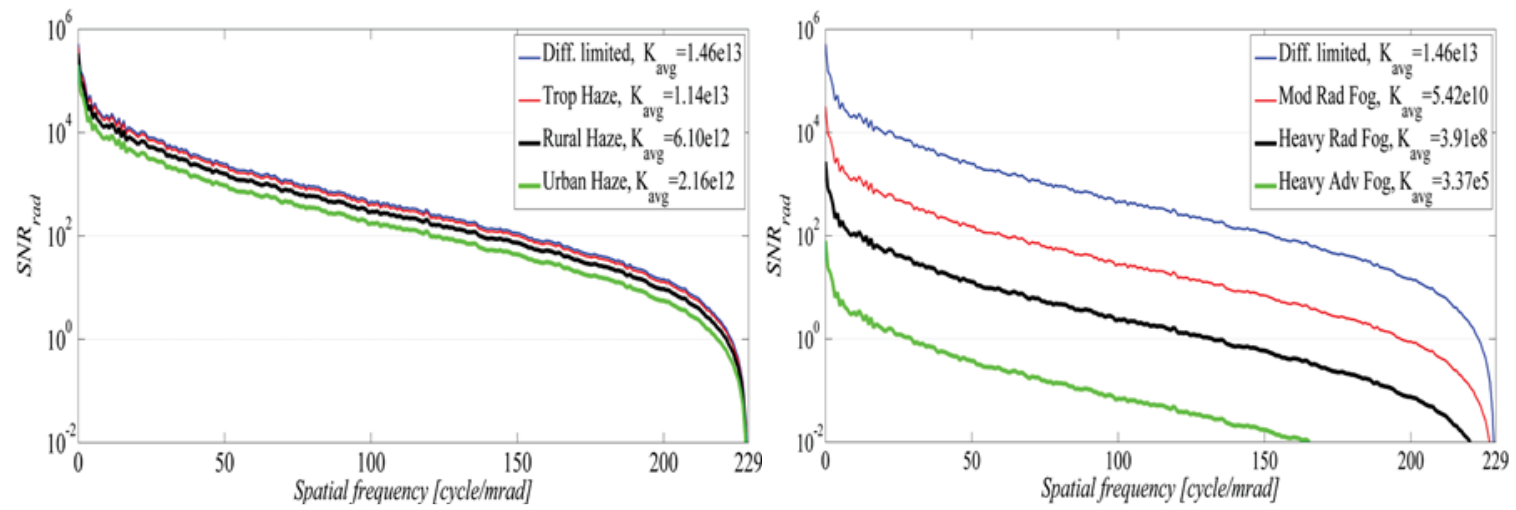

(ii)
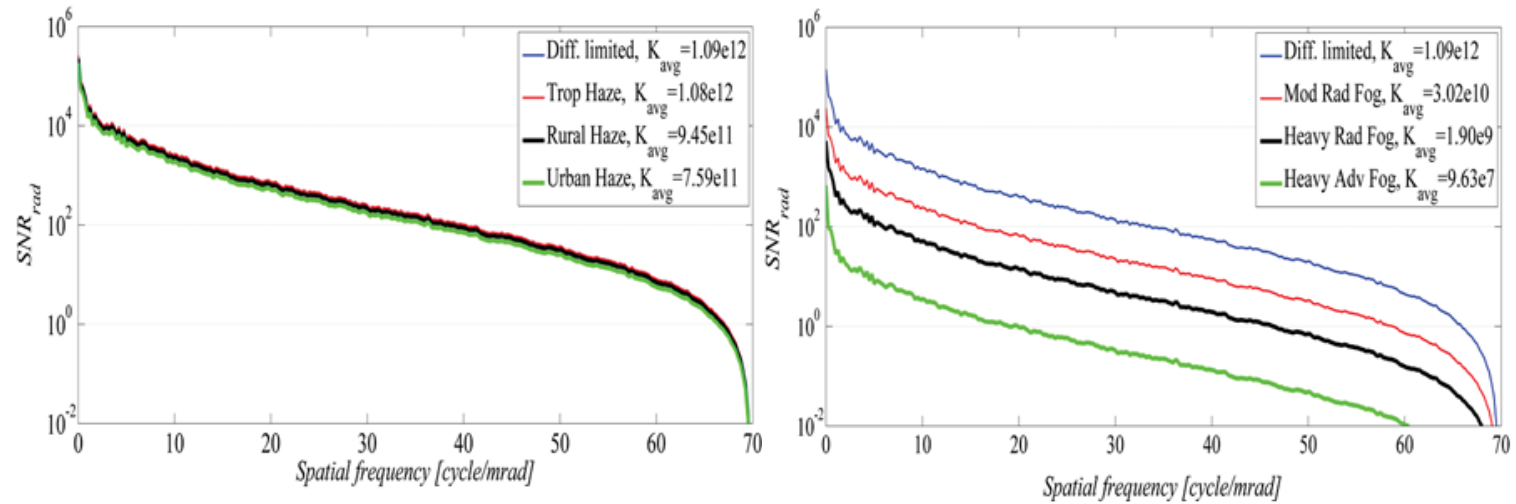

(iii)

(a)

(b)

Figure 3.6 Radially-averaged image spectrum signal-to-noise ratio, $S N R_{\text {rad }}$, for haze and fog aerosol models at the visible, NIR and MIR spectral wavelength bands.

(a) Haze aerosol models, $R=3000[\mathrm{~m}]$, (b) Fog aerosol models, $R=300[\mathrm{~m}]$.

(i) Visible band $\left(\lambda_{c}=0.55 \mu \mathrm{m}\right)$, (ii) NIR band $\left(\lambda_{c}=1.22 \mu \mathrm{m}\right)$, (iii) MIR band $\left(\lambda_{c}=4 \mu \mathrm{m}\right)$. 
Table 3.2 The estimated noise equivalent spatial frequency, $v_{\text {noise }}[\mathrm{cycle} / \mathrm{rad}]$, of the image-spectrum SNR for the atmospheric aerosol models, for each spectral band as shown in figure (3.6), and the corresponding threshold noise effective spatial resolution, $\theta_{\text {th }}[\mathrm{rad}]$.

\begin{tabular}{|c|c|c|c|c|c|c|}
\hline \multirow{2}{*}{$\begin{array}{l}\text { Atmospheric aerosol } \\
\text { models }\end{array}$} & \multicolumn{2}{|c|}{$\begin{array}{c}\text { Visible spectral band } \\
\lambda_{c}=0.55[\mu \mathrm{m}] \\
v_{\text {cut }}=509[\mathrm{cycle} / \mathrm{mrad}]\end{array}$} & \multicolumn{2}{|c|}{$\begin{array}{c}\text { NIR spectral band } \\
\lambda_{c}=1.22[\mu \mathrm{m}] \\
v_{\text {cut }}=229[\mathrm{cycle} / \mathrm{mrad}] \\
\end{array}$} & \multicolumn{2}{|c|}{$\begin{array}{c}\text { MIR spectral band } \\
\lambda_{c}=4[\mu \mathrm{m}] \\
v_{\text {cut }}=70[\mathrm{cycle} / \mathrm{mrad}]\end{array}$} \\
\hline & $\begin{array}{c}v_{\text {noise }} \\
{[\text { cycle/mrad }]}\end{array}$ & $\begin{array}{c}\theta_{t h} \\
{[\mu \mathrm{rad}]}\end{array}$ & $\begin{array}{c}v_{\text {noise }} \\
{[\text { cycle/mrad }]}\end{array}$ & $\begin{array}{c}\theta_{t h} \\
{[\mu r a d]}\end{array}$ & $\begin{array}{c}v_{\text {noise }} \\
{[\text { cycle/mrad }]}\end{array}$ & $\begin{array}{c}\theta_{t h} \\
{[\mu \mathrm{rad}]}\end{array}$ \\
\hline No aerosols & 491 & 2.04 & 223.3 & 4.478 & 67.12 & 14.898 \\
\hline Tropospheric haze & 487 & 2.05 & 223.0 & 4.484 & 67.01 & 14.925 \\
\hline Rural haze & 474 & 2.11 & 221.0 & 4.520 & 66.70 & 14.993 \\
\hline Urban haze & 438 & 2.28 & 218.0 & 4.590 & 66.40 & 15.060 \\
\hline Moderate radiation fog & 428 & 2.34 & 196.0 & 5.102 & 58.76 & 17.018 \\
\hline Heavy radiation fog & 276 & 3.62 & 131.0 & 7.634 & 46.40 & 21.552 \\
\hline Heavy advection Fog & 46 & 21.74 & 24.5 & 40.816 & 19.10 & 52.356 \\
\hline
\end{tabular}

values of $\theta_{t h}$ increase as we transfer from the visible to the MIR band indicating a degradation in the spatial resolution of the imaging system for larger wavelengths and a better spatial resolution for shorter wavelengths. It is also noticed that for each spectral band, the strong small-angle forward scattering that characterizes the fog aerosol models leads to increased number of scattered photons and a reduced number of direct photons reaching the imaging system, consequently the SNR obtained in the image will be reduced significantly for the fog aerosol models, also $\theta_{t h}$ is minimum for the tropospheric haze aerosol model, and $\theta_{t h}$ is maximum for the heavy advection fog aerosol model, indicating a better resolution for the haze aerosol models. This notice is consistent with the values of the meteorological ranges, $M$, listed in table (3.1), for each atmospheric aerosol model at the visible spectral band, where for the haze and fog atmospheric aerosol models, as $M$ decreases, $\theta_{t h}$ increases indicating a reduction in the image 
spectrum SNR. The increased values of $\theta_{t h}$ encountered by fog aerosol models emphasize the degrading effect of fog aerosol models on the spatial resolution of imaging systems operating in the visible, NIR, and MIR spectral bands.

\subsection{Conclusion}

This paper deals with the degrading effect examined by atmospheric scattering media on the level of the signal-to-noise ratio recorded in a detected image and hence, clarifies quantitatively the effect of atmospheric scattering on image quality. The paper presents a radiometric model for estimating the mean numbers of direct and scattered photons detected by an imaging system from an extended target, for a horizontal near-ground imaging scenario, upon propagation through various haze and fog aerosol models. The optical properties of the haze and fog aerosol models are studied based on their particles size distribution functions and spectral refractive indices in the visible, NIR, and MIR spectral bands. We incorporated the optical parameters of the atmospheric aerosol models into the calculation of the effective PSF due to the direct and scattered radiation acquired by an imaging system. The estimation of the photon count is based on calculating two basic criteria: first is the calculation of the radiant power, which reaches the imaging system from an extended target, either being due to a reflected spectral irradiance from the target in the visible and NIR bands, or due to an emitted spectral irradiance in the MIR band, and then propagated through an atmospheric aerosol medium, and second is the calculation of the effective PSF as a function of optical properties of haze and fog 
atmospheric aerosol models. The estimated mean numbers of direct and scattered photons detected by an imaging system are then utilized, to calculate the image spectrum SNR in the visible, NIR, and MIR spectral bands. Finally, we demonstrated a technique for predicting the resolution limit of an imaging system working in the presence of fog and haze atmospheric aerosol models as a function of the optical system, the scattering medium, and the signal level which show a reduction in the value of the noise effective spatial frequency, and consequently an increase in the noise effective spatial resolution, for images taken through fog aerosol media that are characterized by strong forwardscattering, hence emphasize the degrading effect of fog aerosol models on the spatial resolution of imaging systems operating in the visible, NIR, and MIR spectral bands. 


\section{Chapter 4}

\section{Reconstruction of Images Degraded by}

\section{Aerosol Scattering and Measurement}

\section{Noise}

3 The material contained in this chapter was submitted to SPIE Optical Engineering. See Appendix $\mathrm{C}$ for documentation of permission to republish this material. 


\subsection{Introduction}

Absorption and scattering in the atmosphere by haze and aerosols cause blurring in imaging systems which must operate in the presence of these degrading factors $\underline{[1,6,14}$ 15]. In the presence of aerosols in the atmosphere, the point spread function (PSF) of the atmosphere/telescope system in general has the character of a diffraction-limited component which can be attributed to light which passes through the medium without being scattered, plus a blurred "halo" which is due to the scattering medium. Forward scattering has the effect of broadening the angle at which the scattered light arrives at the receiver aperture compared to the unscattered light, and this light is spatially decorrelated from the unscattered light, even though both the scattered and unscattered light originated at the target. We note that turbulence in the atmosphere also causes blurring in imaging by virtue of light propagation through random index of refraction fluctuations caused by turbulent mixing of the air $[2,3]$. Hence, turbulence induced errors are experienced by all of the optical energy reaching the receiver aperture, whereas in the case of aerosol scattering some of the light arriving from the source is unscattered. While it is not strictly the case that aerosol blur and turbulence blur are independent of each other - for example, the atmospheric conditions which give rise to the creation of fog are certainly to some extent affected by the turbulent mixing conditions of the local air mass - we model these two effects as independent, and give our attention here to modeling the effects of aerosol scattering, and inversion techniques to overcome some of the effects of scattering through post detection image reconstruction. This choice is also partially justified by the examination of relatively short paths with relatively strong scatterer 
concentrations. We assume that turbulence effects on the images are dominated by aerosol scattering effects for the work presented here. We note that the average turbulence effects can be incorporated into the model presented here in a straightforward manner by multiplying the long exposure modulation transfer function (MTF) and the MTF derived here for the combination of scattered and unscattered light in the image plane, and this combined MTF can be used to simulate images if desired. The angular spread of light scattered by aerosols is on the order of $\lambda / a_{\text {mode }}[\mathrm{rad}]$, where $\lambda[\mathrm{m}]$ is the propagation wavelength and $a_{\text {mode }}[\mathrm{m}]$ is the modal radius of the size distribution function for the aerosol model $[\mathbf{2 0 , 4 1 ]}$. This ratio is typically on the order of radians, i.e., orders of magnitude greater than the field-of-view of an imaging system. Therefore much of the scattered light by atmospheric aerosols is not incident upon the image sensor and thus reduces the photon flux level available for forming the image. However, small-angle forward scattering, caused by large diameter aerosols, is also present and, when recorded in the image, gives rise to image blur $[\mathbf{1 , 1 5}$. In addition to aerosol-induced blurring, measurement noise effects are also present in observed images, where the limit of detection of images is due to random fluctuations in the arrival times and locations of detected photons which are converted to electrons in a semi-conductor detector. This effect is referred to as photon noise or shot noise, and is represented by Poisson statistics [42]. The image spectrum signal-to-noise ratio (SNR) represents the ratio of the measured light signal to the measurement noise in the spatial frequency domain, and characterizes the image quality and determines the ultimate performance of the imaging system [2]. The spatial frequency that can be recovered in the images that are degraded by aerosolinduced blur and measurement noise is termed noise effective cutoff spatial frequency 
and corresponds to an image spectrum SNR value of 1 . This frequency imposes a limiting bound for the spatial frequency that can be recovered through linear post detection reconstruction algorithms [2]. In this chapter we examine the problem of reconstructing images degraded by aerosol blur and measurement noise. We have previously published a method for analytically predicting the effective PSF of the telescope/atmosphere system, which accurately accounts for the contributions of scattered and unscattered light to the overall PSF, based on the optical properties of the imaging system and the aerosol medium that intercepts the path of light propagation between an observed target and the imaging system [1]. In this chapter representative images of a spoke target are simulated under various conditions of scattering and photon flux levels. The atmospheric aerosol media modeled here are heavy radiation fog, medium advection fog, and heavy advection fog, which are characterized by varying degrees of strong small-angle forward scattering which in turn contribute to varying degrees of image blurring. Inspection of the images presented here shows that a wide range of blurring and noise effects are present for these cases, and in some instances it would be essentially impossible to determine the target in detail from the simulated images. Linear reconstruction of the images is then performed by means of a Wiener filter and a constrained least squares (CLS) filter [43] to help understand the limits of post detection image reconstruction when applied to such data. Our results show that in every case examined some level of additional detail in the target was added by virtue of the image reconstruction processing. However, measurement noise induced image artifacts are also present.

The remainder of this chapter is organized as follows. Using the approach presented in chapter (2), the second section outlines the calculation of the overall effective PSF at the 
visible wavelength band, $\lambda=0.55[\mu \mathrm{m}]$, based on the optical properties of the imaging system and fog aerosol media, at various values of the atmospheric optical depth, $\tau_{\text {atm }}$, and provides the details of the image simulation. Image spectrum SNR calculations are performed using the approach presented in chapter (3), representative images of the spoke target corrupted by fog aerosols-induced blurring and measurement noise are shown, and linear reconstruction of the degraded images by means of a Wiener and a CLS filters is executed in the third section. Conclusions are presented in the fourth section. Our findings generally agree with a previous model that was suggested by Kopeika [15], from the perspective that the atmospheric scattering has a significant impact on the spatial resolution of imaging systems. However, we were not able to reproduce the results of reference [15] regarding aerosol blurring that were achieved at relatively low values of the atmospheric optical depth. Our findings confirm the presence of aerosol-induced blur at extreme conditions of atmospheric turbidity represented by large values of atmospheric optical depth and in this regard our findings match the results presented by Ben Dor [6], and Bissonnette [20].

\subsection{Theory}

Throughout this section we demonstrate the calculation of the effective PSF, as well as the calculation and sampling of the overall MTF based on the optical properties of the fog aerosol models and the imaging system in the visible wavelength band. 


\subsubsection{Effective point spread function}

The calculation of the effective PSF of an imaging system accounts for the effects of the diffraction, scattering, and the appropriate optical power level of both the unscattered (direct) and the scattered radiation arriving at the pupil of the imaging system $[\mathbf{1 , 6}$. Since the scattered radiation is temporally and spatially de-correlated from the direct radiation, we model the effects of the direct (coherent) radiation and the scattered (incoherent) radiation of the aerosol species as additive in the image plane. Atmospheric fog aerosol models are characterized by a narrow peaked phase function, as shown in figure. (4.1), which is due to their relatively large particles size with respect to the wavelength, hence indicating an increased scattered radiation in the forward direction. The radial distribution of the total effective PSF at the image plane of an optical system, $P S F_{t}(r)$, at the given wavelength $\lambda$ is given as the summation of the PSF due to the direct radiation, $P S F_{\operatorname{dir}}(r)$, and the PSF due to the scattered radiation detected by the imaging system, $P S F_{\text {sca }}(r)[\mathbf{1 , 4 , 6 , 2 0}]$,

$$
P S F_{t}(r)=P S F_{d i r}(r)+P S F_{s c a}(r)
$$

where $r[m]$ is the radial distance in the imaging plane. $P S F_{d i r}(r)$ is an Airy disc whose peak is reduced by power losses due to scattering between the target and receiver and is given by [4], 


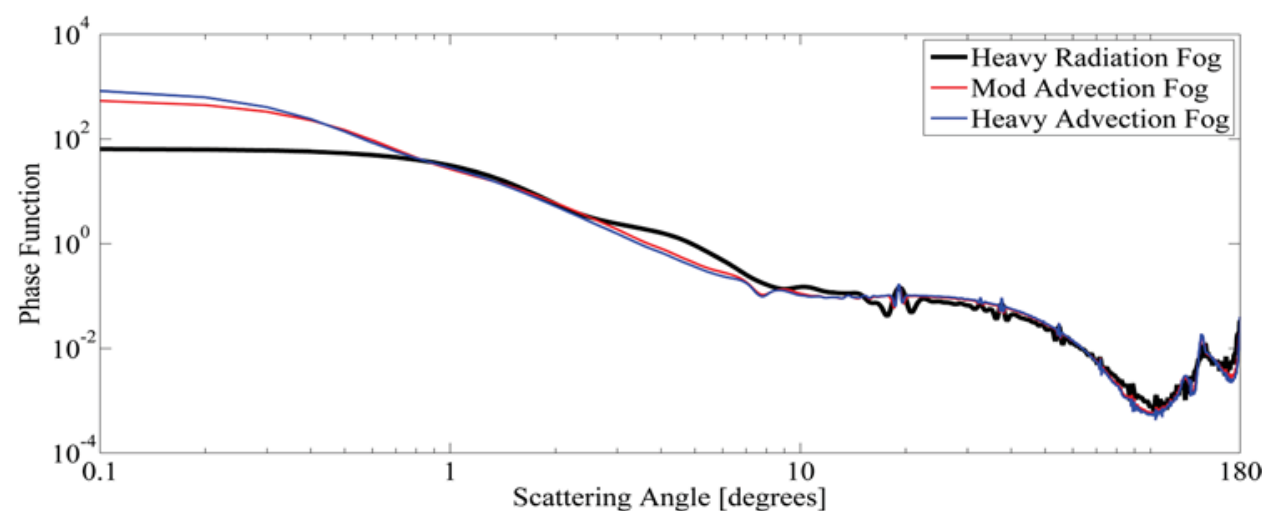

Figure 4.1 Phase function of the atmospheric fog aerosol models at visible wavelength band.

$$
P S F_{d i r}(r)=\exp \left(-\tau_{a t m}\right)\left(\frac{D}{2 r}\right)^{2} J_{1}^{2}\left(\frac{k D r}{2 f}\right)
$$

where $D[m]$ is the diameter of the entrance pupil of the imaging system and $f[m]$ is its focal length, $k\left[\mathrm{~m}^{-1}\right]$ is the wave number of the propagating radiation, $k=2 \pi / \lambda, J_{l}$ is Bessel function of the first kind of order 1 , and $\tau_{\text {atm }}$ [dimensionless] is the optical depth of the atmosphere which is defined as the integral of the extinction coefficient along the path between target and imaging system for a plane-stratified medium [4, 6]. Whereas $P S F_{s c a}(r)$ which represents the scattered radiation that is spread throughout the imaging plane is given by [4],

$$
P S F_{s c a}(r)=\left(\frac{1.64 a_{\text {mode } e} D}{\lambda f \sqrt{R k_{s c a}}}\right) \exp \left[-\tau_{a t m}\left(1-\omega_{o}\right)-\left(\frac{3.28 a_{\text {mode }} r}{\lambda f \sqrt{R k_{s c a}}}\right)\right]
$$


where $k_{s c a}\left[m^{-1}\right]$ is the scattering coefficient, $R[\mathrm{~m}]$ is the range between the target and the imaging system, and $\omega_{o}^{t}$ is the scattering albedo of the medium. The optical properties of the three fog aerosol models examined here in the visible wavelength band, including the absorption coefficient $k_{a b s}\left[\mathrm{~km}^{-1}\right], a_{\text {mode }}, \omega_{o}^{t}$, and $k_{s c a}$ are listed in table (4.1). Considering an imaging system with $D=0.02[\mathrm{~m}]$ and $f=0.1[\mathrm{~m}]$, and according to the optical properties of the fog aerosol models listed in table (4.1) and using Eqs. (4.1 - 4.3), figure (4.2) shows the radial distribution of the normalized total effective PSF at the imaging plane for the fog aerosol models at different values of the range $R$. It is clear that the total PSF is composed of a strong peak at the central portion of the imaging plane, due to the direct un-scattered radiation, and a weaker level halo which is spread across the imaging plane due to the scattered radiation. Note that for each fog model, the level of the scattering halos increases for an increased values of the atmospheric optical depth indicating an increased level of the scattered radiation at the image plane. Moreover for each range heavy advection fog, which is characterized by stronger forward scattering, shows higher level of scattered radiation indicating the influence of more forward scattering on the level of scattered radiation that is acquired by the imaging system.

Table 4.1 Optical properties of fog aerosol models in the visible wavelength band.

\begin{tabular}{ccccc}
$\begin{array}{c}\text { Atmospheric aerosol } \\
\text { models }\end{array}$ & \multicolumn{4}{c}{ Optical properties $(\lambda=0.55 \mu \mathrm{m})$} \\
& $\begin{array}{c}a_{\text {mode }} \\
{[\mu \mathrm{m}]}\end{array}$ & $\omega_{o}{ }^{t}$ & $\begin{array}{l}k_{\text {sca }}(\lambda) \\
{\left[\mathrm{km}^{-1}\right]}\end{array}$ & $\begin{array}{c}k_{\text {abs }}(\lambda) \\
{\left[\mathrm{km}^{-1}\right]}\end{array}$ \\
\hline Heavy radiation fog & 4 & 0.97 & 16.65 & $3.27 \times 10^{-8}$ \\
Moderate advection fog & 8 & 0.98 & 18.75 & $6.61 \times 10^{-8}$ \\
Heavy advection fog & 10 & 0.99 & 28.70 & $1.19 \times 10^{-7}$ \\
\hline
\end{tabular}




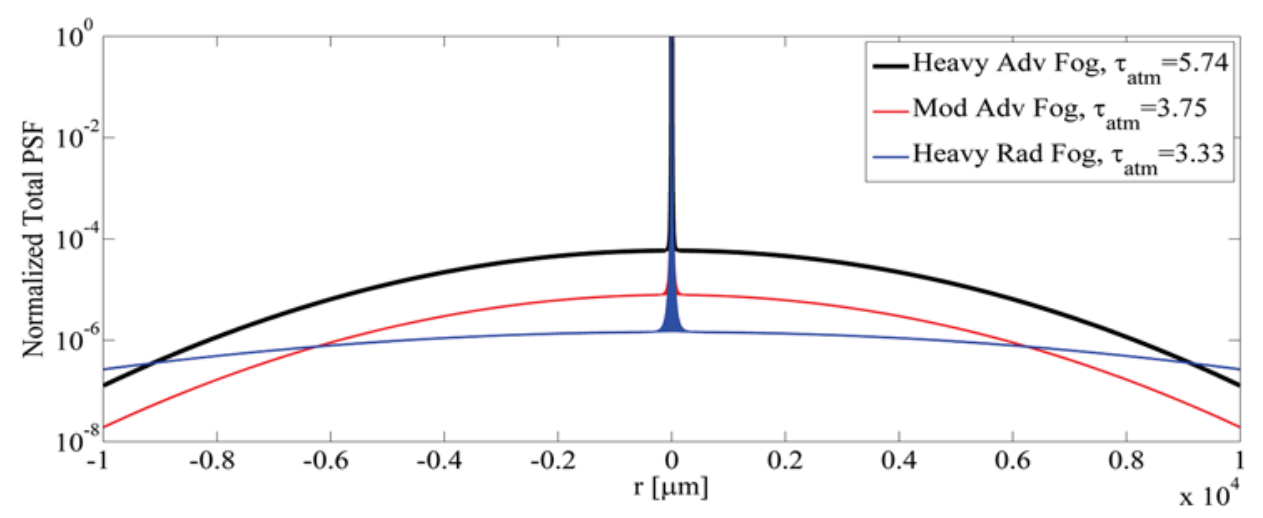

(a)

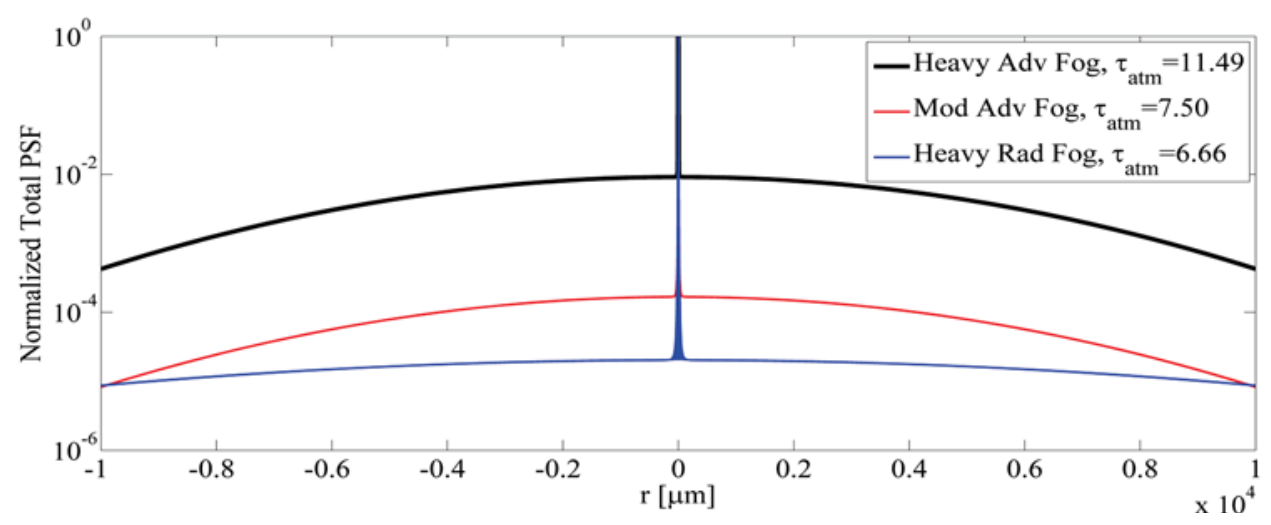

(b)

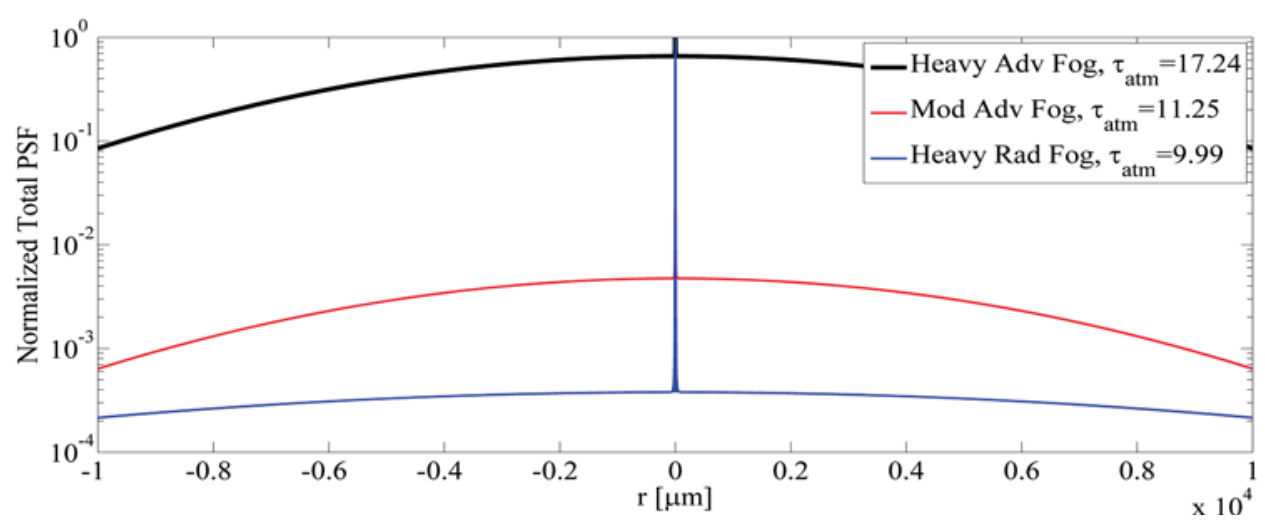

(c)

Figure 4.2 Radial distribution of the normalized total effective PSF for the fog aerosol models at different ranges, $R$, between the target and imaging system values at the visible wavelength.

(a) $R=200[\mathrm{~m}]$, (b) $R=400[\mathrm{~m}]$, (c) $R=600[\mathrm{~m}]$. 


\subsubsection{Modulation transfer function}

The normalized modulus of the two dimensional Fourier transform of the overall PSF gives the total MTF, $M_{T F}[\mathbf{3 2}, \mathbf{4 0}, \mathbf{4 4}]$, which is a metric that describes how the spectral components of the target are affected by the optical properties of the propagation medium and the imaging system,

$$
\operatorname{MTF}_{t}(u, v)=\left|\frac{\int_{-\infty}^{\infty} \int_{-\infty}^{\infty} P S F_{t}(x, y) \times \exp [-j 2 \pi(x u+y v)] d x d y \mid}{\int_{-\infty}^{\infty} \int_{-\infty}^{\infty} P S F_{t}(x, y) d x d y}\right|
$$

where, $x$ and $y[\mathrm{~m}]$ are spatial coordinates at the image plane, and $u$ and $v[$ cycle/rad $]$ are

spatial frequency coordinates in the $x$ and $y$ directions, respectively, where $u=\frac{x}{\lambda f}$ and $v=\frac{y}{\lambda f}\left[\mathrm{rad}^{-1}\right][40]$. The sampling of $M T F_{t}$ is addressed here to satisfy the conditions used in the fast Fourier transform operations. For an image array of size $N$ samples and according to the parameters shown in figure (4.3), the sample spacing in the spatial frequency domain $\Delta v_{\text {img }}\left[\mathrm{rad}^{-1}\right]$ is related to $N$ and the sample spacing in the entrance aperture plane $d x_{p}[\mathrm{~m}]$ by $[\mathbf{4 3}, \mathbf{4 5}]$,

$$
\Delta v_{i m g} \leq \frac{1}{N d x_{p}}
$$




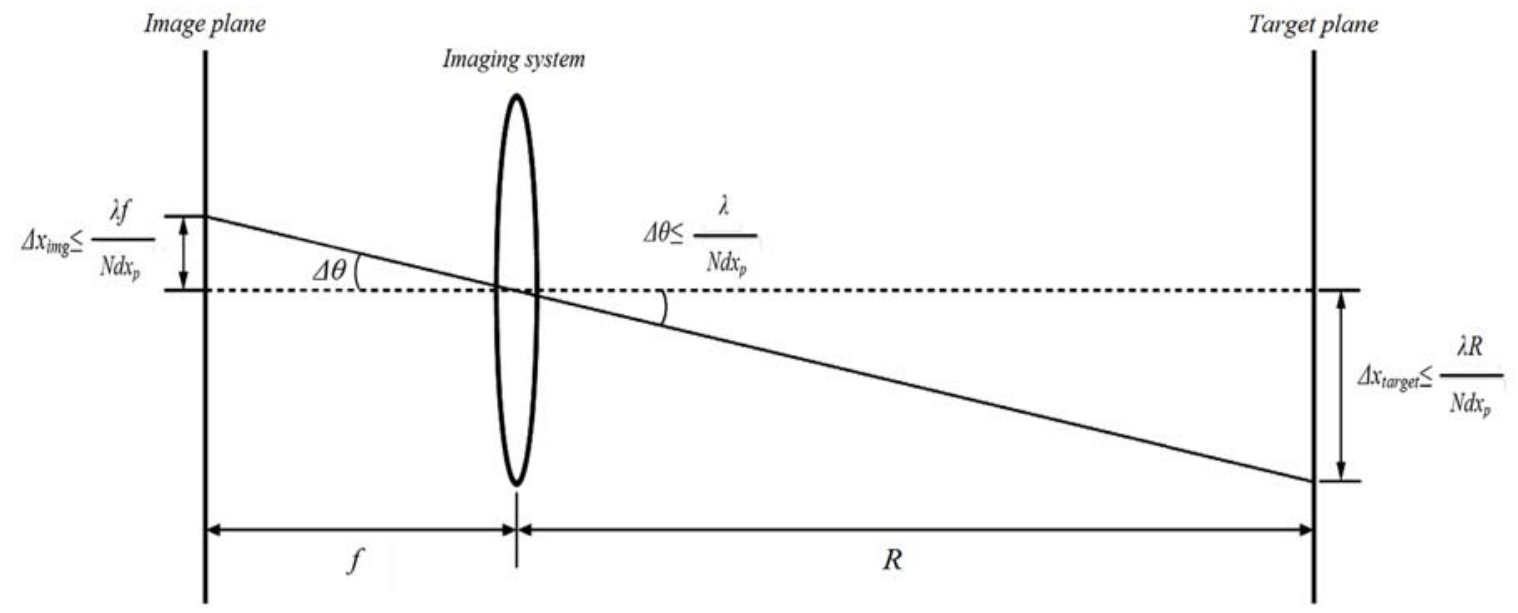

Figure 4.3 Sampling parameters for the total modulation transfer function, $M T F_{t}$, of the fog aerosol models.

Also $\Delta v_{\text {img }}$ is related to the angular sample spacing in the spatial domain $\Delta \theta[\mathrm{rad}]$ by [40],

$$
\Delta v_{i m g}=\frac{\Delta \theta}{\lambda}
$$

then from Eqs. (4.5 - 4.6),

$$
\Delta \theta \leq \frac{\lambda}{N d x_{p}}
$$

Finally the angular spread of $M T F_{t}$ at the image plane, $\theta_{i m g}=N \Delta \theta$, which accounts for the angular extent of the target under observation, $\theta_{\text {target }}[\mathrm{rad}]$, and the angular extent of the overall PSF, $\theta_{P S F}[\mathrm{rad}]$ is given by, 


$$
N \Delta \theta>\theta_{\text {target }}+\theta_{P S F}
$$

where $\theta_{\text {target }}=\frac{L_{\text {target }}}{R}[\mathrm{rad}], L_{\text {target }}[\mathrm{m}]$ is the linear dimension of the target, and

$\theta_{P S F}=\frac{\lambda}{a_{\text {mode }}}[\mathrm{rad}]$. According to the sampling criteria described by Eqs. (4.4 - 4.8),

figure (4.4) shows the properly sampled total MTFs, at visible wavelength, for a diffraction-limited imaging system, and for the heavy radiation, moderate advection and heavy advection fog aerosol models at different ranges $R$ between target and imaging system. Representative simulated images of spoke targets that are corrupted with various fog aerosol models and photon flux levels, and their reconstructions will be shown in the next section.

\subsection{Image simulation and reconstruction}

The calculation of the image spectrum SNR of the reconstructed images gives an estimate for the noise effective cutoff spatial frequency. This frequency imposes a limiting bound for the spatial frequency that can be recovered through linear post detection reconstruction algorithms [2]. The criteria of calculating the image spectrum SNR is presented in section (4.3.1). Simulations of the images corrupted by aerosol scattering and photon noise, as well as linear reconstructions of the degraded images by means of a Wiener filter and a CLS filter followed by calculating the image spectrum SNR of the reconstructed images are performed in section (4.3.2). 


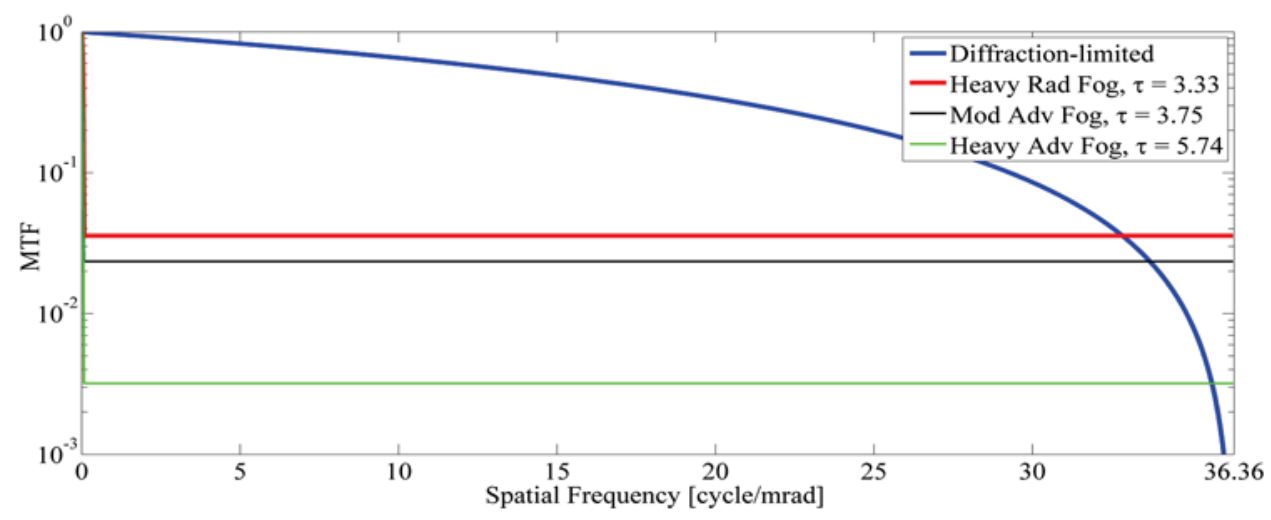

(a)

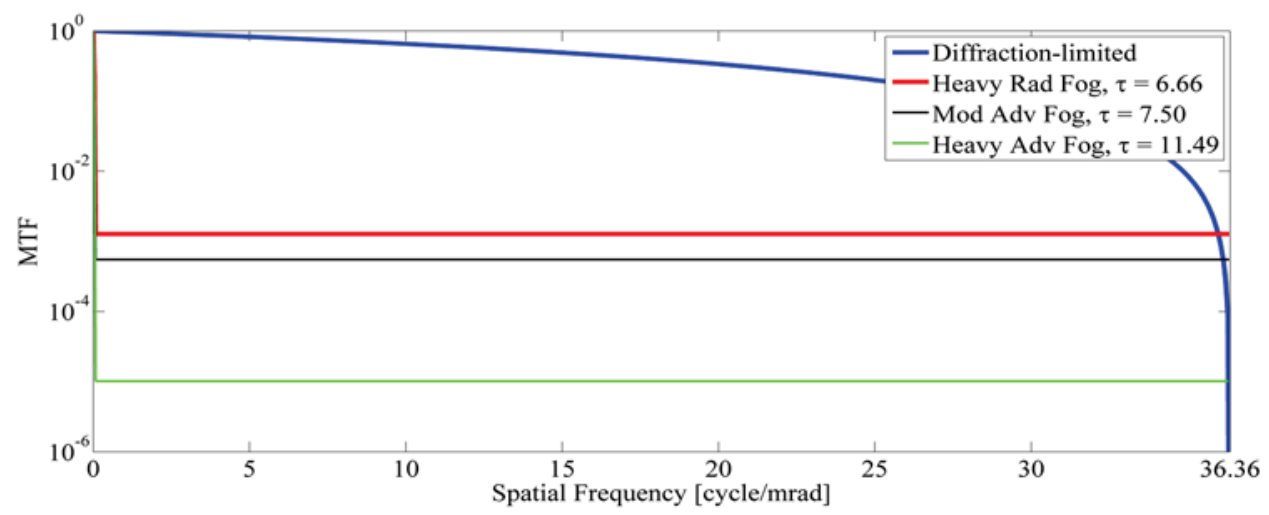

(b)

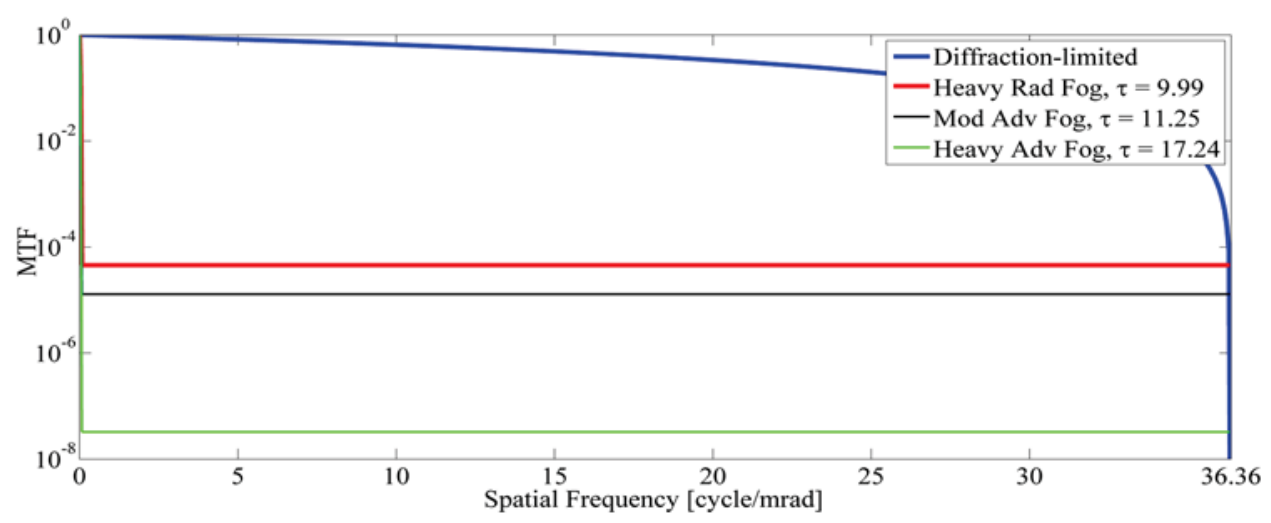

(c)

Figure 4.4 Modulation transfer function, MTF, at visible wavelength, for a diffractionlimited imaging system $M T F_{\text {diff, with }} D=0.02[\mathrm{~m}]$ and $f=0.1[\mathrm{~m}]$, and for atmospheric fog aerosol models, $M T F_{t}$, at different ranges, $R$, between target and imaging system. (a) $R=200[\mathrm{~m}]$, (b) $R=400[\mathrm{~m}]$, (c) $R=600[\mathrm{~m}]$. 


\subsubsection{Image spectrum signal-to-noise ratio}

let $I(v, v)$ be the Fourier transform of an image which has been detected with a photon counting camera. Image spectrum SNR is defined as the ratio of the mean of $I(v, v)$ to the standard deviation of $I(v, v)$. Hence, the image spectrum SNR is a measure of the relative strengths of the image spectrum signal and the random variability of the signal in the spatial frequency domain, and provides a useful metric of evaluating the effects of various sources of noise on the image quality. The image spectrum SNR, $\operatorname{SNR}(u, v)$, of images obtained with a deterministic MTF and a photon-limited image detection system is given by [2],

$$
\operatorname{SNR}(u, v)=\frac{K_{d i r}\left|\operatorname{MTF}_{t}(u, v) F_{n}(u, v)\right|}{\sqrt{K_{d i r}+K_{s c a}}}
$$

where $F_{n}(v)$ is the normalized Fourier spectrum of the target irradiance distribution, or simply the target spectrum, and $K_{d i r}$ and $K_{s c a}$ are the numbers of direct and scattered photons arriving at the entrance aperture of the imaging system, respectively. Estimates of $K_{d i r}$ and $K_{s c a}$ may be calculated by,

$$
K_{d i r}=\left[\frac{P S F_{d i r}(0)}{P S F_{t}(0)}\right] K_{a v g}
$$




$$
K_{s c a}=\left[\frac{P S F_{s c a}(0)}{P S F_{t}(0)}\right] K_{a v g}
$$

where $P S F_{\text {dir }}(0), P S F_{\text {sca }}(0), P S F_{t}(0)$, are the direct, scattered, and total PSFs at the central portion of the imaging plane, respectively, and $K_{a v g}$ is the average number of photons detected at the detector plane of the imaging system, which can be calculated using radiance theorem [37],

$$
K_{a v g}=\frac{A A_{a p e r} \tau_{a t m}(\lambda) \tau_{o p} \eta t_{\mathrm{int}} \lambda \int_{\lambda} \rho E_{\lambda} d \lambda}{\pi h c R^{2}}
$$

where $A$ is the target area $\left[m^{2}\right], A_{\text {aper }}$ is the entrance aperture area $\left[m^{2}\right], \tau_{o p}$ is optics transmittance, $\eta$ is quantum efficiency of the detectors, $\rho$ is target reflectance, $t_{\text {int }}$ is integration time for the detectors $[s], E_{\lambda}$ is the spectral irradiance reflected from the target [W $\left.\mathrm{m}^{-2} \mu \mathrm{m}^{-1}\right], h$ is Planck's constant, $h=6.626 \times 10^{-34}[\mathrm{~J} . \mathrm{s}]$, and $c$ is the speed of light, $c=3 \times 10^{8}[\mathrm{~m} / \mathrm{s}]$. The noise effective cutoff spatial frequency $v_{\text {noise }}[\mathrm{cycle} / \mathrm{rad}]$ is defined as the spatial frequency that corresponds to an image spectrum SNR value of 1. vnoise represents a limiting bound for the spatial frequency that can be recovered through linear post detection reconstruction algorithms [2]. The reciprocal of $v_{\text {noise }}$ indicates a threshold noise effective spatial resolution, $\theta_{t h}[\mathrm{rad}]$. 


\subsubsection{Image reconstruction}

The simulation of images corrupted by atmospheric scattering due to various fog aerosols, and photon noise is performed by multiplying the spectrum of the target by the total MTF and then superimposing Poisson noise $N(u, v)$ according to the photons flux levels [43],

$$
G(u, v)=M T F_{t}(u, v) \times F_{n}(u, v)+N(u, v)
$$

where $G(u, v)$ is the spectrum of the degraded image. In our simulation we use a spoke target, with diameter $L_{\text {target }}=2[\mathrm{~m}]$, located at different ranges $R$ from the imaging system. Figure (4.5) shows simulations of the images after being corrupted with aerosol scattering and photon noise. We notice from figure (4.5) that the spatial resolution is degraded severely for extremely turbid atmosphere represented by large values of atmospheric optical depth. The linear post detection reconstruction algorithms used here to reconstruct the degraded images shown in figure (4.5) include Wiener filter and CLS filter. The estimated reconstructed image $\hat{F}(u, v)$ is given by [43],

$$
\hat{F}(u, v)=M(u, v) \times G(u, v)
$$

where $M(u, v)$ is the restoring filter function. The restoring function of Wiener filter, $M_{\text {Wiener, }}$ is given by [43], 

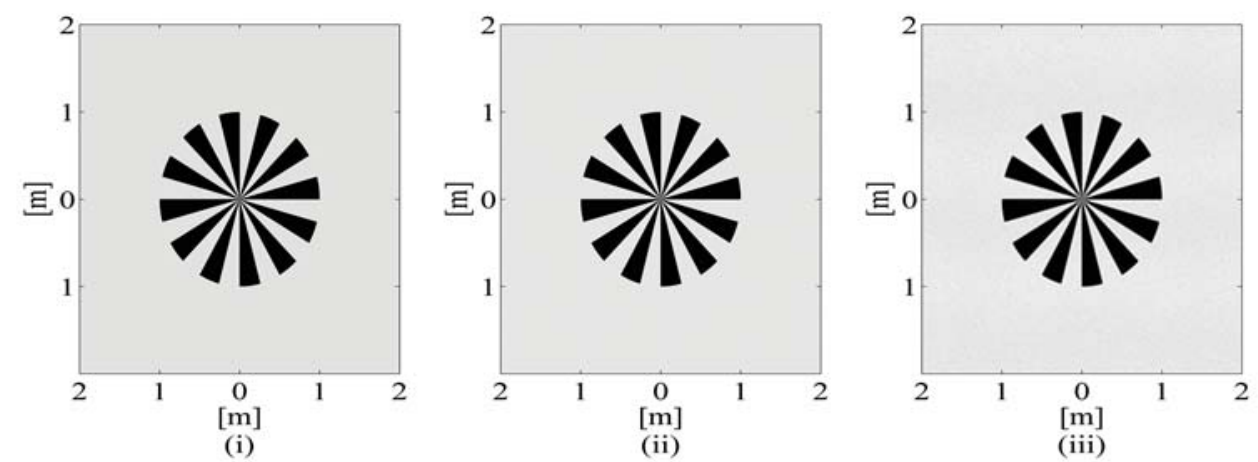

(a)

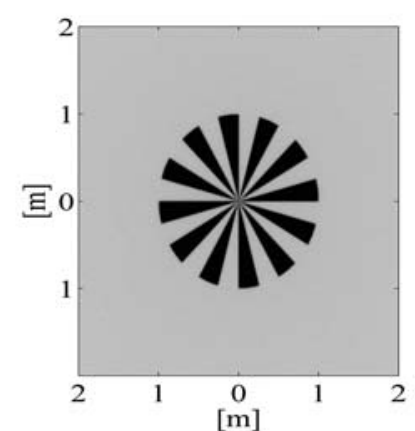

(i)

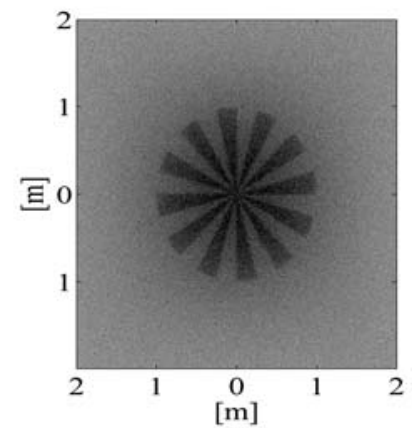

(i)

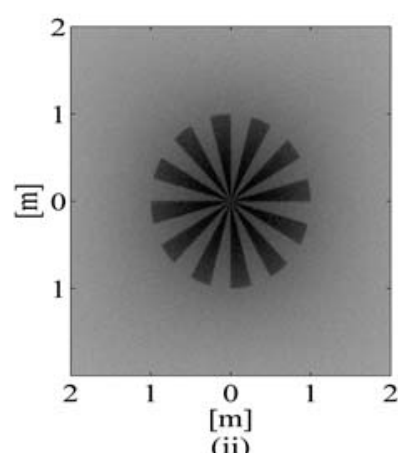

(b)
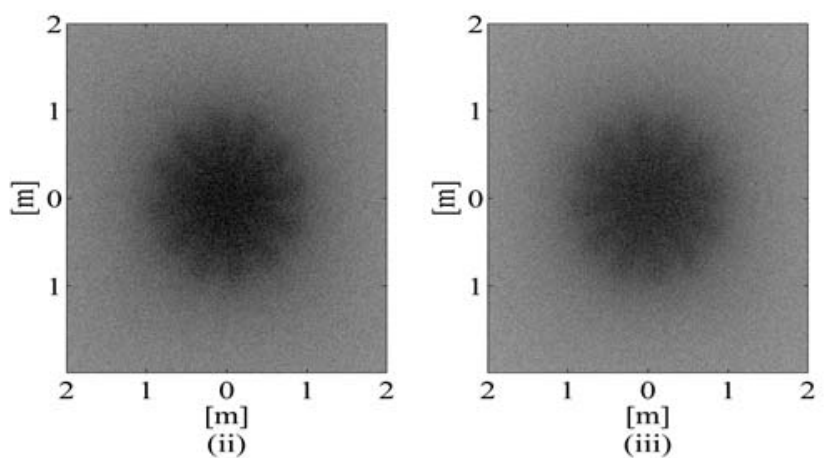

(c)

Figure 4.5 Images degraded by scattering due to atmospheric fog aerosol models, and corrupted with Poisson noise at different ranges, $R$, between target and imaging system.

(a) $R=200[\mathrm{~m}]$, (b) $R=400[\mathrm{~m}]$, (c) $R=600[\mathrm{~m}]$.

(i) Heavy radiation fog, (ii) Moderate advection fog, (iii) Heavy advection fog. 


$$
M_{\text {Wiener }}=\frac{1}{\operatorname{MTF}_{t}(u, v)} \frac{\left|\operatorname{MTF}_{t}(u, v)\right|^{2}}{\left|\operatorname{MTF}_{t}(u, v)\right|^{2}+K}
$$

where $K$ is a specified constant which is chosen interactively to yield the best visual results [43]. Whereas the restoring function of CLS filter, $M_{C L S}$, is given by [43],

$$
M_{C L S}=\frac{\operatorname{MTF}_{t}^{*}(u, v)}{\left|\operatorname{MTF}_{t}(u, v)\right|^{2}+\gamma|P(u, v)|^{2}}
$$

where $\operatorname{MTF}_{t}{ }^{*}(u, v)$ is the complex conjugate of $M T F_{t}(u, v), P(u, v)$ is the Fourier transform of the Laplacian matrix [43],

$$
p(x, y)=\left[\begin{array}{ccc}
0 & -1 & 0 \\
-1 & 4 & -1 \\
0 & -1 & 0
\end{array}\right]
$$

and $\gamma$ is a scalar that is evaluated iteratively $[43,46]$. Figures (4.6) and (4.7) show the reconstructed images after applying a Wiener filter and a CLS filter, respectively, on the degraded images of figure (4.5). In order to make an estimate of the spatial resolution that can be restored, we calculated the image spectrum SNR of the reconstructed images based on the criteria explained in section (4.3.1). Figure (4.8) shows the radially-averaged image spectrum SNR, $S N R_{r a d}$, versus spatial frequency for the reconstructed images given 


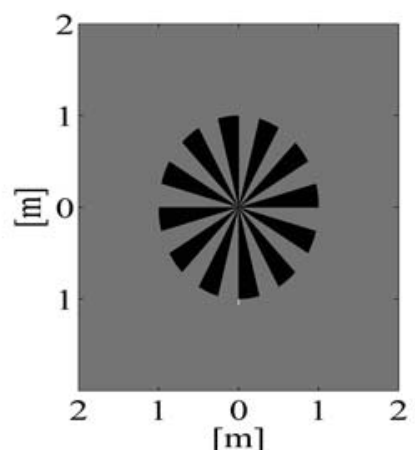

(i)

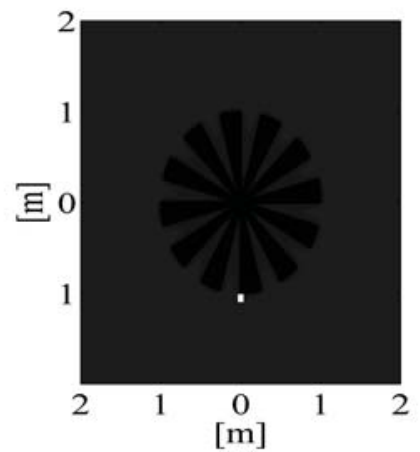

(i)

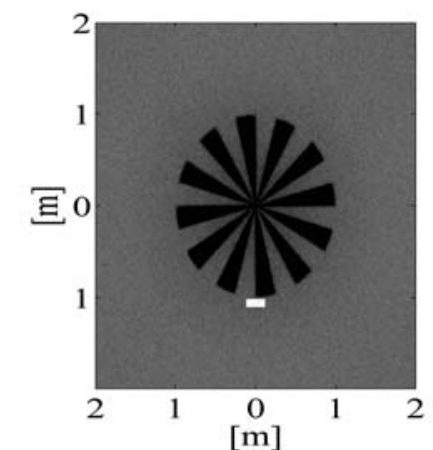

(i)

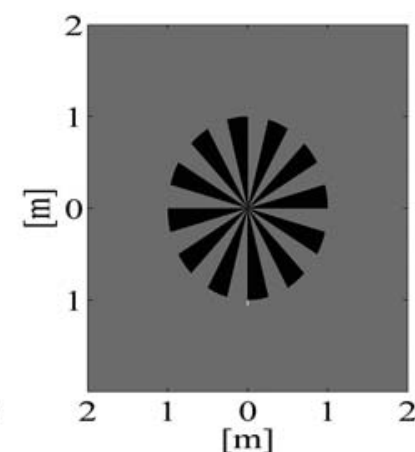

(ii)

(a)

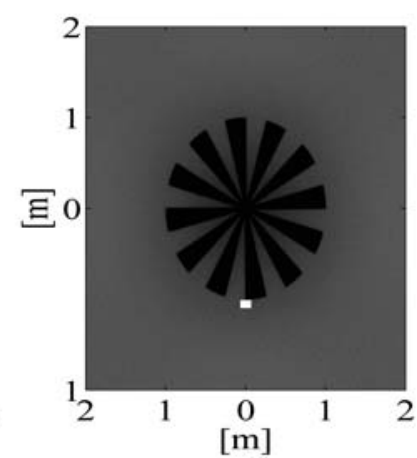

(ii)

(b)

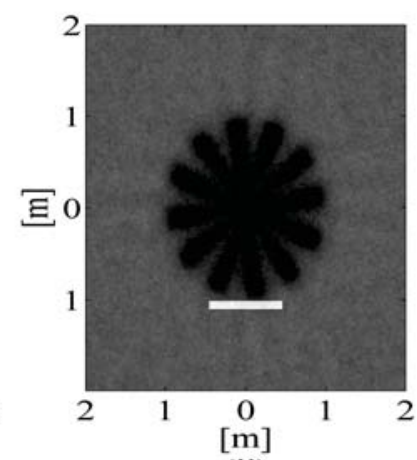

(ii)

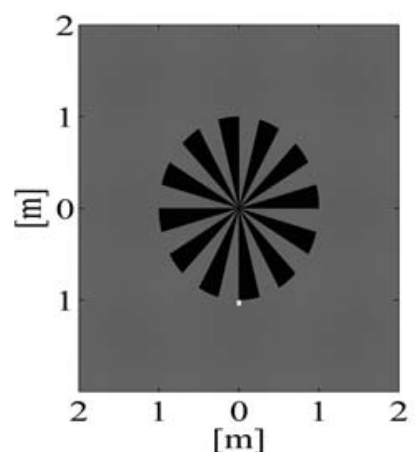

(iii)

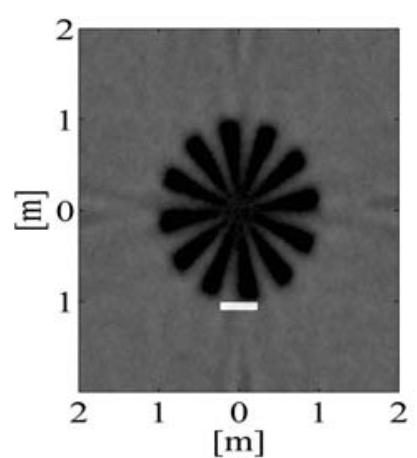

(iii)

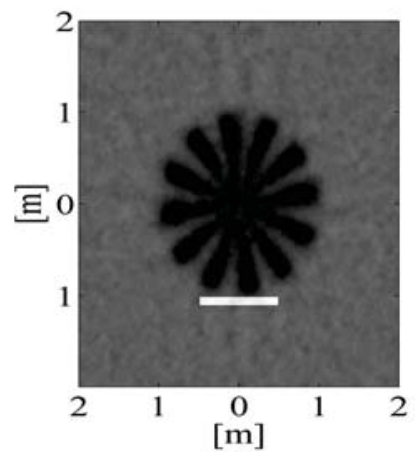

(iii)

(c)

Figure 4.6 Reconstruction of degraded images of figure (4.5) using Wiener filter.

(a) $R=200[\mathrm{~m}]$, (b) $R=400[\mathrm{~m}]$, (c) $R=600[\mathrm{~m}]$.

(i) Heavy radiation fog, (ii) Moderate advection fog, (iii) Heavy advection fog. 


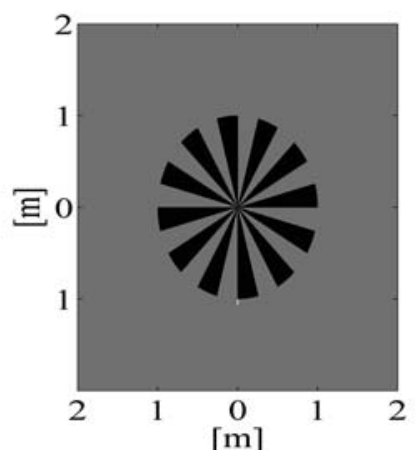

(i)

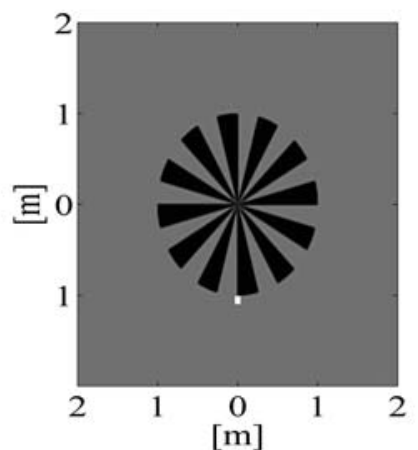

(i)

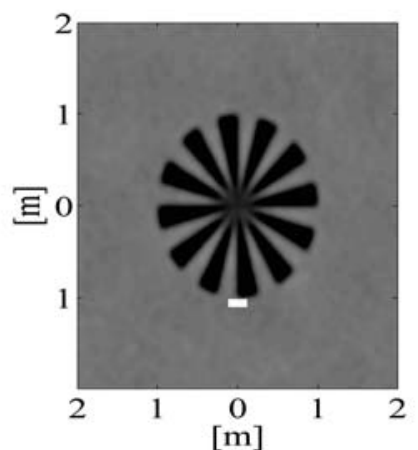

(i)

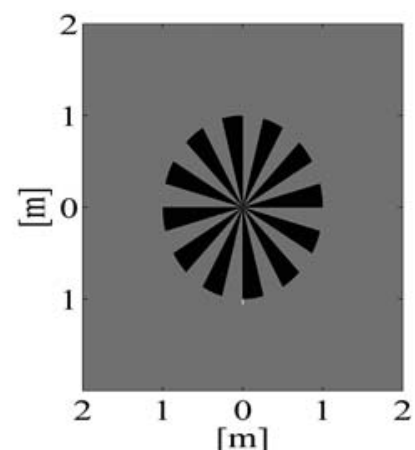

(ii)

(a)

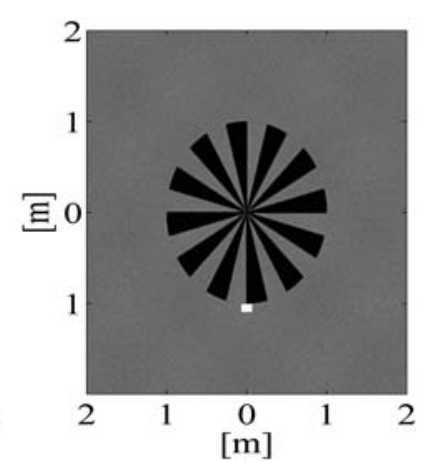

(ii)

(b)

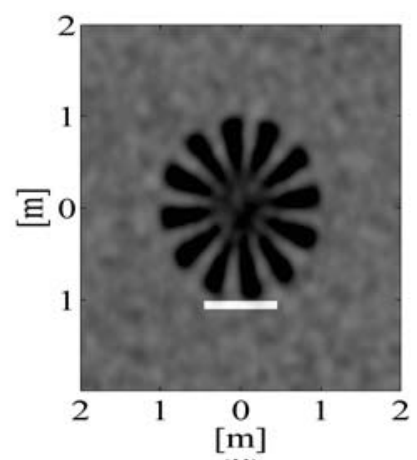

(ii)

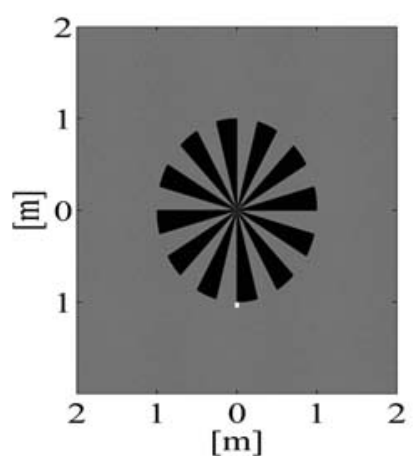

(iii)

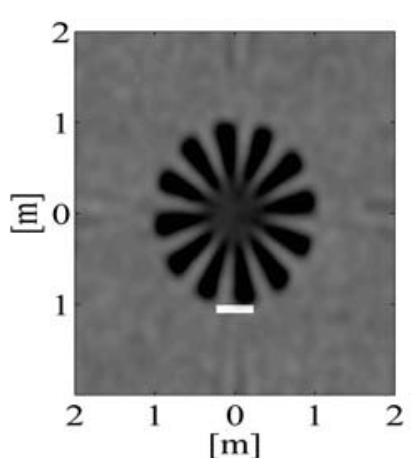

(iii)

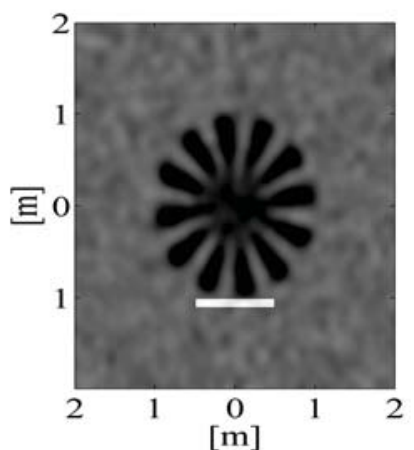

(iii)

(c)

Figure 4.7 Reconstruction of degraded images of figure (4.5) using constrained least squares (CLS) filter.

(a) $R=200[\mathrm{~m}]$, (b) $R=400[\mathrm{~m}]$, (c) $R=600[\mathrm{~m}]$.

(i) Heavy radiation fog, (ii) Moderate advection fog, (iii) Heavy advection fog. 
in figures (4.5) and (4.6). Table (4.2) lists values of the noise effective cutoff spatial frequency, $v_{n o i s e}$, that are extracted from figure (4.8) and the corresponding values of the noise effective spatial resolution, $\theta_{t h}$. The product $\theta_{t h} R$ gives an estimated value of the spatial resolution which can be restored after applying the reconstruction filters. The estimated values of the restored spatial resolution are listed in table (4.2), and the corresponding distance in the image plane is represented on figures (4.6) and (4.7) by white bars below the reconstructed spoke target. The size of these white bars indicate the best spatial resolution that can be reconstructed by means of Wiener and CLS filters, and are shown to be consistent with the reconstructions that are shown in figures (4.6) and (4.7). Although noise artifacts are present in the reconstructions, they achieve a very close estimate of the original target. Results of reconstructions show an excellent recovered spatial resolution of the images corrupted with aerosol blur and measurement noise at relatively small atmospheric optical depths to within the limits imposed by MTF attenuation caused by the optics, the atmosphere, and measurement noise. Whereas for badly blurred images acquired in extremely turbid fog aerosol media, which are represented by large optical depths, some level of the details of the original spoke targets have been recovered by virtue of the image reconstruction processing despite the presence of blur and noise induced artifacts. This is attributed to the important conclusion that the spatial resolution of images degraded by aerosol scattering and measurement noise can be restored up to the limit imposed by the noise effective spatial resolution of the measurement. 


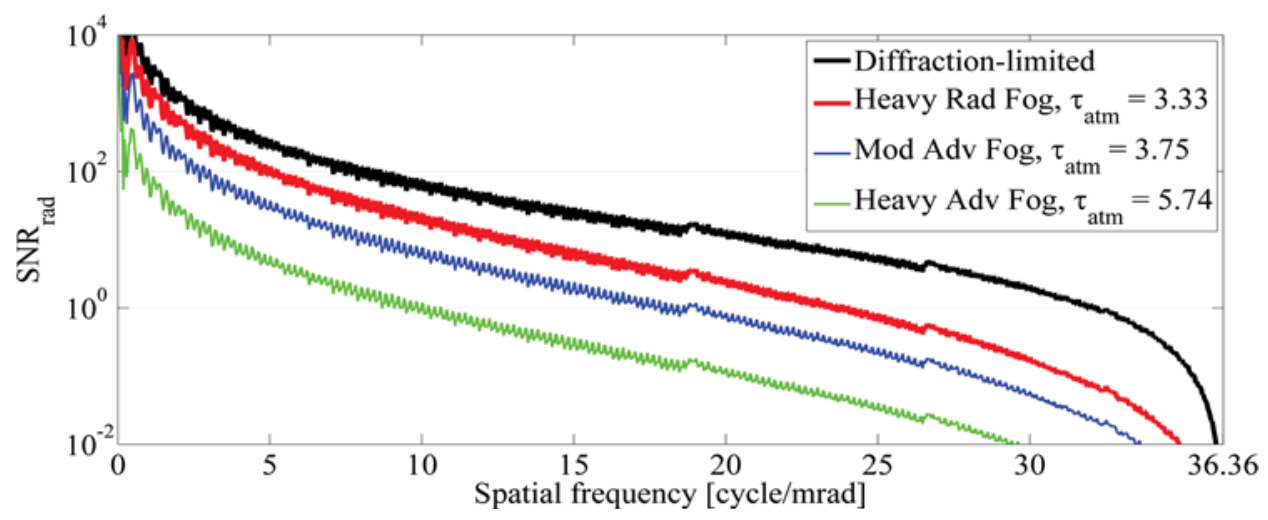

(a)

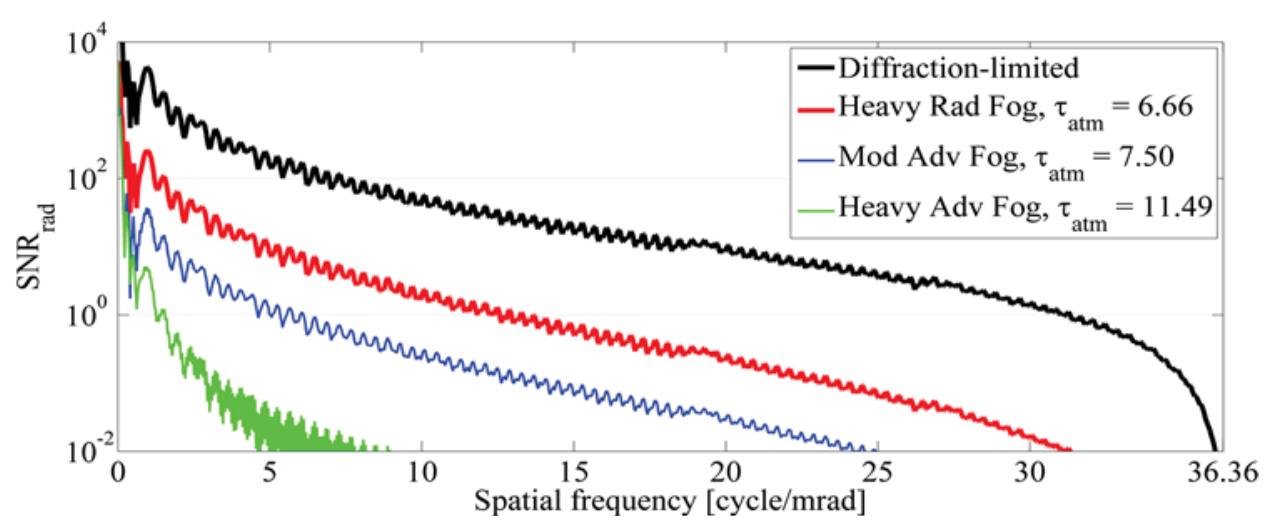

(b)

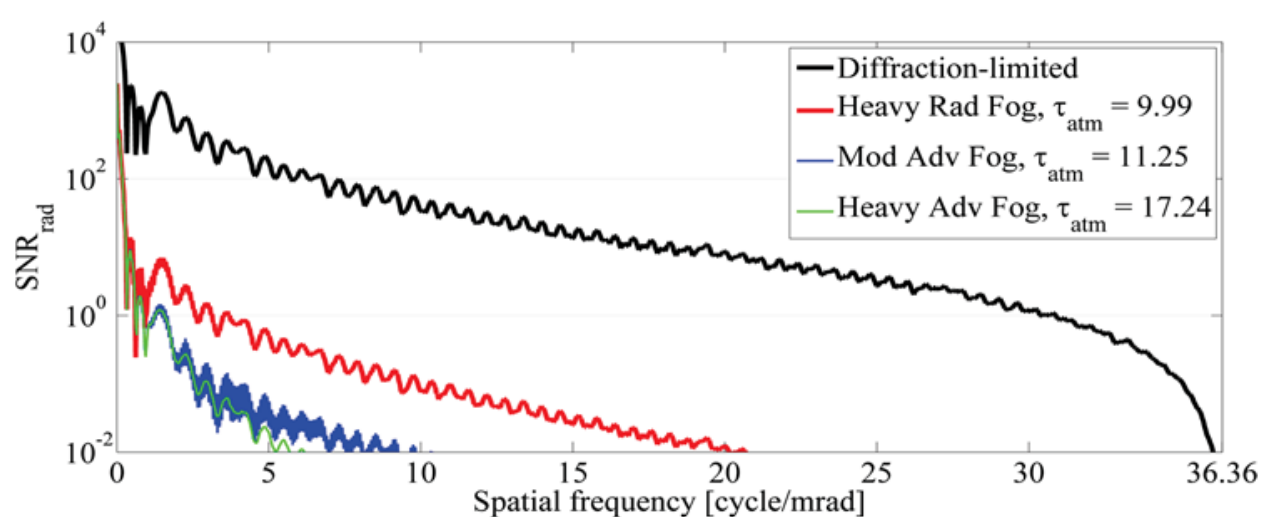

(c)

Figure 4.8 Reconstruction of degraded images of figure (4.6) using constrained least squares (CLS) filter.

(a) $R=200[\mathrm{~m}]$, (b) $R=400[\mathrm{~m}]$, (c) $R=600[\mathrm{~m}]$.

(i) Heavy radiation fog, (ii) Moderate advection fog, (iii) Heavy advection fog. 
Table 4.2 The estimated noise effective cutoff spatial frequency $v_{n o i s e}[\mathrm{cycle} / \mathrm{rad}]$ of the image spectrum SNR shown in figure (4.8), the corresponding threshold noise effective spatial resolution $\theta_{t h}[\mathrm{rad}]$, and the values of the restored spatial resolution $[\mathrm{m}] ; \theta_{t h} R$.

\begin{tabular}{|c|c|c|c|c|c|c|c|c|c|}
\hline \multirow{2}{*}{$\begin{array}{l}\text { Fog } \\
\text { aerosol } \\
\text { models }\end{array}$} & \multicolumn{3}{|c|}{$R=200[\mathrm{~m}]$} & \multicolumn{3}{|c|}{$R=400[\mathrm{~m}]$} & \multicolumn{3}{|c|}{$R=600[\mathrm{~m}]$} \\
\hline & $\begin{array}{c}v_{\text {noise }} \\
\text { [cycle/mrad] }\end{array}$ & $\begin{array}{c}\theta_{t h} \\
{[\mu r a d]}\end{array}$ & $\begin{array}{l}\theta_{\text {th }} R \\
{[\mathrm{~cm}]}\end{array}$ & $\begin{array}{c}v_{\text {noise }} \\
{[\text { cycle/mrad }]}\end{array}$ & $\begin{array}{c}\theta_{t h} \\
{[\mu r a d]}\end{array}$ & $\begin{array}{l}\theta_{t h} R \\
{[\mathrm{~cm}]}\end{array}$ & $\begin{array}{c}v_{\text {noise }} \\
\text { [cycle/mrad }]\end{array}$ & $\begin{array}{c}\theta_{t h} \\
{[\mu r a d]}\end{array}$ & $\begin{array}{l}\theta_{\text {th }} R \\
{[\mathrm{~cm}]}\end{array}$ \\
\hline $\begin{array}{c}\text { Heavy } \\
\text { Radiation }\end{array}$ & 23.27 & 42.97 & 0.86 & 11.82 & 84.60 & 3.38 & 2.58 & 387.59 & 23.26 \\
\hline $\begin{array}{l}\text { Moderate } \\
\text { Advection }\end{array}$ & 17.31 & 57.78 & 1.16 & 4.66 & 214.59 & 8.58 & 0.66 & 1515.15 & 90.91 \\
\hline $\begin{array}{c}\text { Heavy } \\
\text { Advection }\end{array}$ & 8.91 & 112.23 & 2.24 & 0.92 & 1086.9 & 43.48 & 0.62 & 1612.90 & 96.77 \\
\hline
\end{tabular}

\subsection{Conclusion}

The reduction in image quality is attributed either to the reduction of image contrast, due to atmospheric aerosols that cause extinction of the propagating radiation and scattering of such radiation at large enough angles leading to an ultimate reduction in SNR, or to image blurring, due to small-angle forward scattering by atmospheric aerosols, where the scattered radiation collected by the sensor will be slightly deviated such that fine image features such as lines and edges are blurred. This paper quantifies atmospheric scattering and introduces an approach for reconstruction of images distorted by atmospheric aerosol blur and measurement noise for a horizontal imaging scenario, using estimates of the effective PSF which account for unscattered and scattered radiation detected by the imaging system and a superimposed photon noise, which is attributed to the inherent random fluctuations of the incident photon flux. Representative images of a spoke target corrupted by fog aerosols-induced blurring and measurement noise show a severe loss of 
spatial resolution for increased values of the atmospheric optical depth. We attribute this degradation to the strong small-angle forward scattering, which characterizes fog aerosol models, beside the increasing measurement noise which reduces the SNR obtained in the image. Although noise artifacts are present in the reconstructions, they achieve a very close estimate of the original target. Results of reconstructions show a complete recovered spatial resolution of the images corrupted with aerosol blur and measurement noise at relatively small atmospheric optical depths to within the limits imposed by MTF attenuation caused by the optics, the atmosphere, and the measurement noise, whereas for badly blurred images acquired in extremely turbid fog aerosol media, which are represented by large optical depths, only part of the details of the original spoke targets have been recovered despite the presence of blur and noise artifacts. Analysis of the image spectrum SNR of the reconstructed images shows that images degraded by aerosol scattering and measurement noise can be reconstructed, and the spatial resolution can be recovered up to the limit imposed by the noise effective cutoff spatial frequency of the measurement. 


\section{Chapter 5}

\section{Conclusions}

In this dissertation, the problem of light scattering by haze and fog atmospheric aerosol models was addressed in a way that enables us to understand the limits imposed by an imaging system, scattering medium, and the signal level on reconstruction of images degraded by aerosol-induced blur and measurement noise. In chapter (2) a detailed overview of light scattering by haze and fog aerosol models was done using Mie scattering theory. A detailed electro-magnetic model was developed that calculates the optical properties of a single spherical particle, also the physical parameters of various haze and fog atmospheric aerosol models, including their particle compositions and size distribution functions, were incorporated to calculate the optical properties of these atmospheric media. This detailed model was used to compute the average point spread function of the atmosphere/telescope system, which is the basic physical function that describes the spatial response of an imaging system. In chapter (3) a novel model for estimating the image spectrum signal-to-noise ratio for a horizontal imaging scenario through scattering media was proposed based on two criteria: the effective PSF calculations presented in chapter (2), and a radiometric model that estimates the average number of photons detected by an imaging system from an extended target. This model considered both the reflected radiation from an extended target in the visible and near 
infra-red (NIR) bands, and the emitted radiation from the target in the mid infra-red (MIR) band. Chapter (4) examined the problem of reconstructing images degraded by aerosol-induced blur and measurement noise using estimates of the effective PSF that was introduced in chapter (2), and image spectrum SNR considerations, that were investigated in chapter (3). Representative images of a spoke target acquired under various conditions of scattering and photon flux levels were simulated and linear reconstruction algorithms were applied on the degraded images.

\subsection{Summary of key results}

The work presented in this dissertation makes a number of important contributions to the field of atmospheric optics:

First, a detailed electro-magnetic model was developed to calculate the effective PSF of an imaging system. This model showed the significant effect of atmospheric scattering on

the contrast and spatial resolution of images acquired by imaging systems, due to the increased level of the scattered radiation PSF and the reduced level of the direct radiation PSF upon increasing the atmospheric optical depth.

Second, we adopted a novel model for the calculation of the image spectrum SNR that predicts the resolution limit of an imaging system working in the presence of fog and haze as a function of the optical system, the scattering medium, and the signal level. This model provides a means of estimating the noise effective cutoff spatial frequency of an 
imaging system which imposes a limit on the highest spatial frequency which can be reconstructed with a post detection image reconstruction algorithm. We concluded a reduction in the value of the noise effective cutoff spatial frequency for images taken through fog aerosol media, hence emphasized the degrading effect of fog aerosol models on the spatial resolution of imaging systems which operate in the visible, NIR, and MIR spectral bands.

Finally, results of reconstruction of images degraded by aerosol-induced blurring and measurement noise effects, which is examined using estimates of the overall PSF and image spectrum SNR considerations, show that the spatial resolution can be recovered in badly blurred images up to the limit imposed by the noise effective cutoff spatial frequency of the measurement.

\subsection{Suggestions for future work}

As with any research, there are still some questions that this dissertation did not answer and may be of interest to other researchers. The most important issue that made a great debate among the researchers working in the field of atmospheric optics in general and in atmospheric scattering in particular is the way an imaging system detects and measures a scattered radiation. We tried to answer this question through introducing our novel model that links the different components that constitute the imaging chain: the optical system, the scattering medium, and the measured signal level. 
Some future ideas related to our work involve the incorporation of optical turbulence effects to our scattering model. The resulting model may be used on a larger scale to improve the quality of images acquired through a scattering and turbulent atmospheric media. Another future direction is the practical implementation of our model to reconstruct real scaterring-degraded images. Also, an advanced trend is to make a real time reconstruction in the visible and infrared spectral bands. 


\section{References}

1. M. E. Hanafy, M. C. Roggemann, and D. O. Guney, "Detailed effects of scattering and absorption by haze and aerosols in the atmosphere on the average point spread function of an imaging system," J. Opt. Soc. Am. A 31, 1312 - 1319 (2014).

2. M. C. Roggemann and B. M. Welsh, Imaging Through Turbelence. (CRC Press, 1996).

3. J. W. Goodman, Statistical Optics. (J. Wiley \& Sons, Inc, 2000).

4. A. Ishimaru, Wave Propagation and Scattering in Random Media. (IEEE Press and Oxford University Press, New York, 1997).

5. R. F. Lutomirski, "Atmospheric degradation of electro-optical system performance," Appl. Opt. 17, 3915-3921 (1978).

6. B. Ben Dor, A. D. Devir, G. Shaviv, P. Bruscaglioni, P. Donelli, and A. Ismaelli, "Atmospheric scattering effect on spatial resolution of imaging systems," J. Opt. Soc. Am. A 14, 1329-1337 (1997).

7. P. N. Reinersman and K. L. Carder, "Monte Carlo simulation of the atmospheric point-spread function with an application to correction for the adjacency effect," Appl. Opt. 34, 4453 - 4471 (1995).

8. D. Toublanc, "Henyey Greenstein and Mie phase functions in Monte Carlo radiative transfer computations," Appl. Opt. 35, 3270 - 3274 (1996). 
9. J. Colombi and K. Louedec, "Monte Carlo simulation of light scattering in the atmosphere and effect of atmospheric aerosols on the point spread function," J. Opt. Soc. Am. A 30, $2244-2252$ (2013).

10. R. A. Leathers, T. V. Downes, C. O. Davis, and C. D. Mobley, "Monte Carlo Radiative Transfer Simulations for Ocean Optics: A Practical Guide, " (Naval Research Laboratory, Naval Research Laboratory, Washington, DC 20375-5320, 2004).

11. H.C. van de Hulst, Light scattering by small particles. (Dover Publications, Inc. , New York, 1981).

12. D. Deirmendjian, Electromagnetic Scattering on Spherical Polydispersions. (American Elsevier Publishing Company, Inc., New York, 1969).

13. E.P. Shettle and R.W. Fenn, "Models for the Aerosols of the Lower Atmosphere and the Effects of Humidity Variations on Their Optical Properties, " AFGL-TR-79-0214 (Air Force Geophysics Laboratory, Hanscom Air Force Base, MA, 1979).

14. C. F. Bohren and D. R. Huffman, Absorption and Scattering of Light by Small Particles. (Wiley-VCH, 2004).

15. N. S. Kopeika and D. Sadot, "Imaging through the atmosphere: practical instrumentation-based theory and verification of aerosol modulation transfer function," J. Opt. Soc. Am. A 10, 172-179 (1993).

16. N. S. Kopeika and D. Sadot, "Imaging through the atmosphere: practical instrumentation-based theory and verification of aerosol modulation transfer function: reply to comment," J. Opt. Soc. Am. A 12, 1017 - 1023 (1995). 
17. N. S. Kopeika, I. Dror, and D. Sadot, "Causes of atmospheric blur: comment on Atmospheric scattering effect on spatial resolution of imaging systems," J. Opt. Soc. Am. A 15, 3097 - 3106 (1998).

18. N. S. Kopeika and D. Arbel, "Imaging through the atmosphere: an overview," SPIE 3609, 78-89 (1999).

19. I. Dror and N. S. Kopeika, "Experimental comparison of turbulence modulation transfer function and aerosol modulation transfer function through the open atmosphere," J. Opt. Soc. Am. A 12, 870-980 (1995).

20. L. R. Bissonnette, "Imaging through fog and rain," Opt. Eng. 31, 1045-1052 (1992).

21. W. J. Wiscombe, "Improved Mie scattering algorithms," Appl. Opt. 19, 1505-1509 (1980).

22. R. C. Shirkey and D. H. Tofsted, "High Resolution Electro-Optical Aerosol Phase Function Database PFNDAT2006, " ARL-TR-3877 (U.S. Army Research Laboratory, White Sands Missile Range, 2008).

23. S. G. Gathman, "Optical properties of the marine aerosol as predicted by the Navy aerosol model," Opt. Eng. 22, 57-62 (1983).

24. E.P. Shettle D.R. Longtin, J.R. Hummel, J.D. Pryce "A Wind Dependent Desert Aerosol Model: Radiative Properties, " AFGL-TR-88-0112 (Air Force Geophysics Laboratory: Hanscom Air Force Base, MA, 1988).

25. K. N. Liou. (2002). An Introduction to Atmospheric Radiation (Second ed.).

26. R. G. Grainger, "Some Useful Formulae for Aerosol Size Distributions and Optical Properties, " (University of Oxford, 2012). 
27. M. T. Eismann and D. A. LeMaster, "Aerosol modulation transfer function model for passive long-range imaging over a nonuniform atmospheric path," Opt. Eng. 52, 046201-1-046201-13 (2013).

28. E.P.Shettle F.X.Kneizys, L.W.Abreu, J.H.Chetwynd, G.P.Anderson, J.E.A.Selby, W.O.Gallery, \& S.A.Clough, "User's Guide to LOWTRAN 7, " AFGL-TR-88-0177 (Air Force Geophysics Laboratory: Hanscom Air Force Base, MA, 1988).

29. Y. Kuga and A. Ishimaru, "Modulation transfer function of layered inhomogeneous random media using the small-angle approximation," Applied Optics 25, 4382 4385 (1986).

30. Y. Kuga and A. Ishimaru, "Modulation transfer function and image transmission through randomly distributed spherical particles," J. Opt. Soc. Am. A 2, 2330 - 2335 (1985).

31. W. H. Wills, "Loss of Resolution in Water as a Result of Multiple Small-Angle Scattering," J. Opt. Soc. Am. 59, 686 - 691 (1968).

32. P. Bruscaglioni, P. Donelli, A. Ismaelli, and G. Zaccanti, "Monte Carlo calculations of the modulation transfer function of an optical system operating in a turbid medium," Appl. Opt. 32, 2813 - 2824 (1993).

33. GEO/GF, "Solar Spectrum, " EUM/MTG/DEF/10/0611 EUMETSAT, 2010).

34. A. T. Mecherikunnel and J. C. Richmond, "Spectral Distribution of Solar Radiation, " TM 82021 National Aeronautics and Space Administration, Goddard Space Flight Center, Greenbelt, Maryland 20771, 1980).

35. B. A. Bodhaine, N. B. Wood, E. G. Dutton, and J. R. Slusser, "On Rayleigh Optical Depth Calculations," J. of Atm. and Ocean. Tech. 16, 1854-1861 (1999). 
36. K. N. Liou, An Introduction to Atmospheric Radiation. (Academic Press, 2002).

37. R. W. Boyd, Radiometry and the Detection of Optical Radiation. (John Wiley \& Sons, Inc., 1983).

38. R. C. Shirkey and S. G. O’Brien, "An Analysis of Atmospheric Aerosol Scattering for Mid-Infrared Systems, " ARL-TR-3938 (Army Research Laboratory White Sands Missile Range, NM 88002-5501, 2006).

39. L. R. Bissonnette, "Multiscattering model for propagation of narrow light beams in aerosol media," Applied Optics 27, 2478-2484 (1988).

40. J. W. Goodman, Introduction to Fourier Optics, Third ed. (Roberts \& Company 2005).

41. Y. Yitzhaky, "Restoration of atmospherically blurred images according to whetherpredicted atmospheric modulation transfer function," Opt. Eng. 36, 3064 - 3072 (1997).

42. A. Foi, M. Trimeche, V. Katkovnik, and K. Egiazarian, "Practical PoissonianGaussian Noise Modeling and Fitting for Single-Image Raw-Data," IEEE TRANSACTIONS ON IMAGE PROCESSING 17, 1737 - 1754 (2008).

43. R. C. Gonzales and R. E. Woods, Digital Image Processing. (Pearson Education, Inc., 2008).

44. O. K. Ersoy, Diffraction, Fourier Optics and Imaging. (John Wiley \& Sons, Inc., Hoboken, New Jersey, 2007).

45. M. C. Roggemann and T. J. Schulz, "Algorithm to increase the largest aberration that can be reconstructed from Hartmann sensor measurements," Appl. Opt. 37, 4321 $4329(1998)$. 
46. D. Sadot, S. R. Rotman, and N. S. Kopeika, "Comparison between high-resolution restoration techniques of atmospherically distorted images," Opt. Eng. 34, 144 - 153 (1994). 


\section{Appendix A}

Mie scattering theory 
The scattering and absorption of electro-magnetic (EM) radiation by atmospheric aerosols depend on the aerosols optical properties including the EM cross sections, single scattering albedo, scattering phase function, and asymmetry parameter. This appendix explores the application of Mie scattering theory to calculate the optical properties for a single homogeneous spherical particle, with a certain size and refractive index. For a particle to cause scattering, the particle must have a different index of refraction than the medium in which it exists. Consider a spherical particle with radius $r$ that is illuminated by a plane harmonic wave as shown in figure (A.1). The direction of propagation of the incident wave defines the z-axis. The forward direction $\hat{e}_{z}$ and the scattering direction $\hat{e}_{r}$ define the scattering plane. $E_{i \theta}$ and $E_{i \varphi}$ are the parallel and orthogonal components of the incident electric field to the scattering plane respectively, whereas $E_{s \theta}$ and $E_{s \varphi}$ are the parallel and orthogonal components of the scattered electric field respectively. Mie scattering theory solves Maxwell's equations inside and outside the spherical particle, and by applying the boundary conditions that imply continuity of the incident field components $E_{i \theta}$ and $E_{i \varphi}$ across the sphere boundary, we get the scattered far-field components $E_{s \theta}$ and $E_{s \varphi}[4,11,14]$

$$
\begin{gathered}
E_{\| s}=E_{S \theta}=\frac{i e^{i k z}}{k r} S_{2}(\theta) \cos \phi \\
E_{\perp \mathrm{s}}=E_{S \phi}=-\frac{i e^{i k z}}{k r} S_{1}(\theta) \sin \phi
\end{gathered}
$$




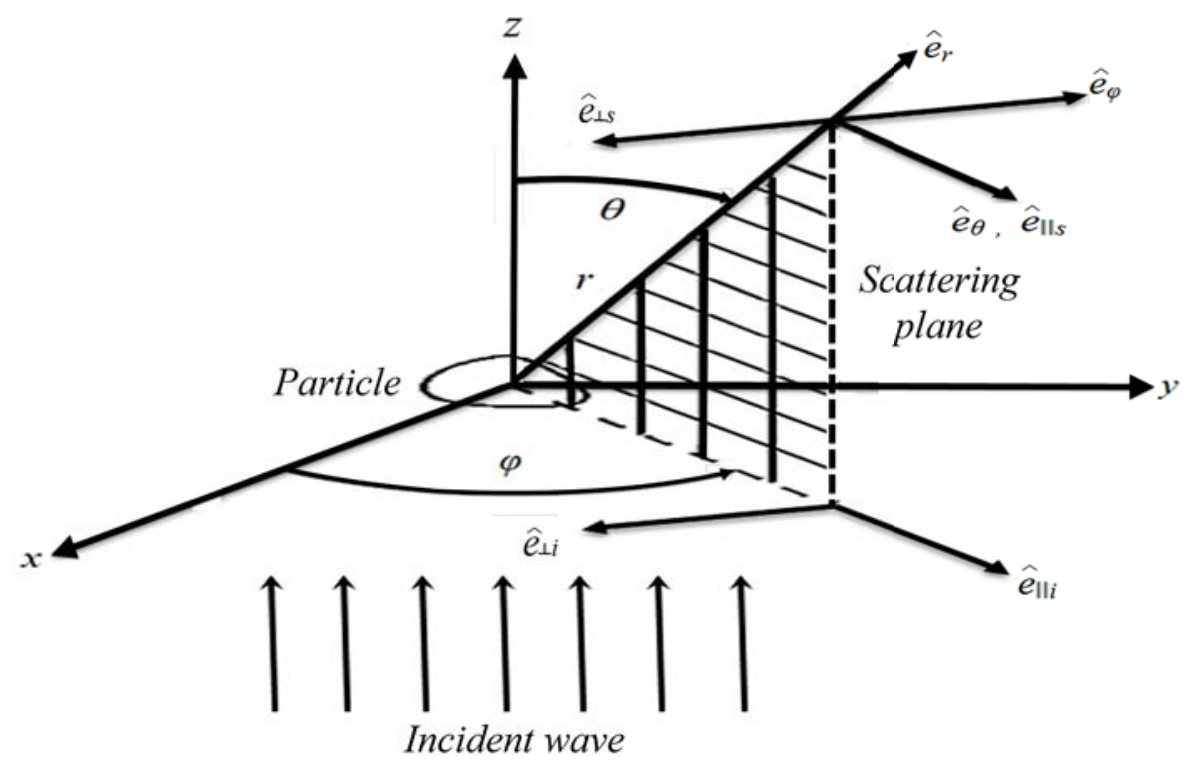

Figure A.1 Geometry for scattering of an electromagnetic wave by a spherical particle.

where $k=2 \pi / \lambda$ is the wave number $\left[m^{-1}\right], z$ is the range from the scattering sphere to an observation point in the scattering plane $[m]$, and $S_{1}(\theta)$ and $S_{2}(\theta)$ are the scattering amplitude functions. Due to the fact that scattering by spherical particles is always uniform in azimuth angle $\varphi$, the scattering amplitude functions and all other scattering functions are always written simply as a function of polar scattering angle $\theta . S_{1}(\theta)$ and $S_{2}(\theta)$ are described by the following infinite series $[4,11,14]$,

$$
\begin{aligned}
& S_{1}(\theta)=\sum_{n=1}^{\infty} \frac{(2 n+1)}{n(n+1)}\left[a_{n} \pi_{n}(\cos \theta)+b_{n} \tau_{n}(\cos \theta)\right] \\
& S_{2}(\theta)=\sum_{n=1}^{\infty} \frac{(2 n+1)}{n(n+1)}\left[a_{n} \tau_{n}(\cos \theta)+b_{n} \pi_{n}(\cos \theta)\right]
\end{aligned}
$$


where $a_{n}$ and $b_{n}$ are Mie coefficients, and $\pi_{n}(\cos \theta)$ and $\tau_{n}(\cos \theta)$ are angle-dependent functions describing the angular scattering patterns of the spherical harmonics and are given by [14],

$$
\begin{gathered}
\pi_{n}(\cos \theta)=\frac{P_{n}^{1}(\cos \theta)}{\sin \theta} \\
\tau_{n}(\cos \theta)=\frac{d}{d \theta}\left[P_{n}^{1}(\cos \theta)\right]
\end{gathered}
$$

where $P_{n}{ }^{l}(\cos \theta)$ are the associated Legendre functions of the first kind of degree $n$ and order $1 . \pi_{n}(\cos \theta)$ and $\tau_{n}(\cos \theta)$ can be computed following the recurrence relations,

$$
\begin{gathered}
\pi_{n}=\left(\frac{2 n-1}{n-1} \cos \theta\right) \pi_{n-1}-\left(\frac{n}{n-1}\right) \pi_{n-2} \\
\tau_{n}=(n \cos \theta) \pi_{n}-(n+1) \pi_{n-1}
\end{gathered}
$$

which begin with $\pi_{0}=0, \pi_{1}=1, \pi_{2}=3 \cos \theta, \tau_{0}=0, \tau_{1}=\cos \theta$ and $\tau_{2}=3 \cos (2 \theta)$ [12].

Figure (A.2) shows plots of the angle-dependent functions $\pi_{n}(\cos \theta)$ and $\tau_{n}(\cos \theta)$ versus $\theta$. 


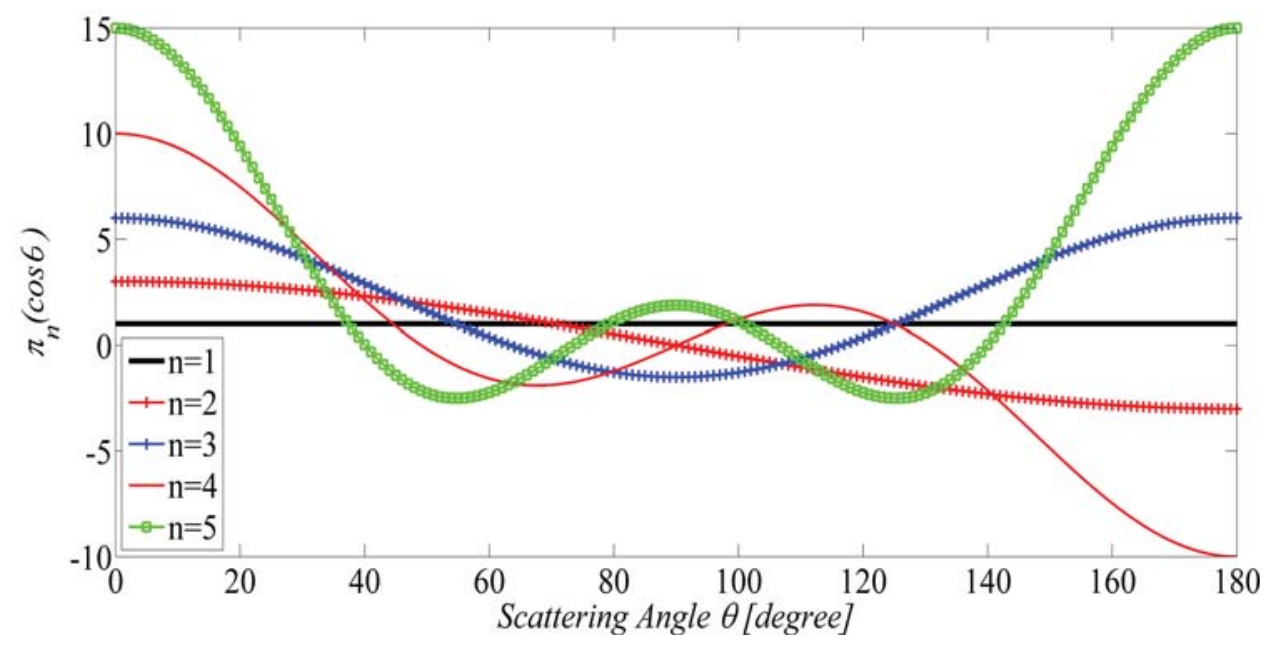

(a)

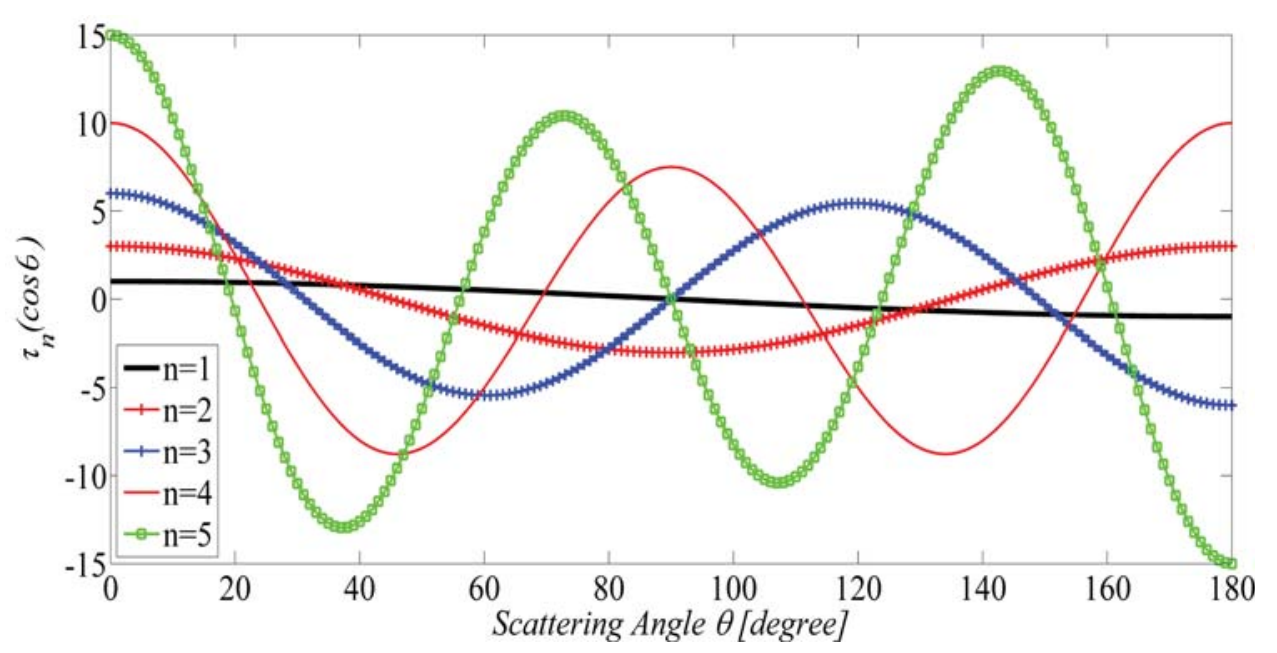

(b)

Figure A.2 Angle-dependent functions $\pi_{n}(\cos \theta)$ and $\tau_{n}(\cos \theta)$ used in calculating the scattering amplitude functions $S_{1}(\theta)$ and $S_{2}(\theta)$.

(a) $\pi_{n}(\cos \theta)$, (b) $\tau_{n}(\cos \theta)$.

Whereas, $a_{n}$ and $b_{n}$ are given by [14], 


$$
b_{n}=\frac{\mu_{1} j_{n}(m x)\left\{\frac{d}{d x}\left[x j_{n}(x)\right]\right\}-j_{n}(x)\left\{\frac{d}{d(m x)}\left[m x j_{n}(m x)\right]\right\}}{\mu_{1} j_{n}(m x)\left\{\frac{d}{d x}\left[x h_{n}^{(1)}(x)\right]\right\}-h_{n}^{(1)}(x)\left\{\frac{d}{d(m x)}\left[m x j_{n}(m x)\right]\right\}}
$$

where $x$ is the size parameter for a particle with radius $r$ and is given by,

$$
x=k r=\frac{2 \pi r}{\lambda}
$$

$m$ is the complex refractive index of the sphere relative to the refractive index of the ambient medium; $m=m^{\prime}+i m^{\prime \prime}$, Both $m^{\prime}$ and $m^{\prime \prime}$ depend on the wavelength of the propagating radiation. $\mu_{1}$ is the ratio of the magnetic permeability of the sphere to the magnetic permeability of the ambient medium. $h_{n}^{(1)}(z)=j_{n}(z)+i y_{n}(z)$, where $h_{n}^{(1)}(z)$ is spherical Bessel function of the third kind of order $n$ (sometimes called spherical Hankel function), $j_{n}(z)$ and $y_{n}(z)$ are spherical Bessel functions of the first and second kind respectively, of order $n$ and of given arguments, $z=x$ or $z=m x$. Spherical Bessel functions are related to Bessel functions by the following relationships [4],

$$
\begin{aligned}
& j_{n}(z)=\sqrt{\frac{\pi}{2 z}} J_{n+0.5}(z) \\
& y_{n}(z)=\sqrt{\frac{\pi}{2 z}} Y_{n+0.5}(z)
\end{aligned}
$$


where $J_{n}$ and $Y_{n}$ are Bessel functions of the first and second kind respectively of order $n$. Mie coefficients $a_{n}$ and $b_{n}$ are the most challenging part in Mie scattering theory computations and once calculated, all measurable quantities associated with scattering and absorption such as EM cross sections and scattering matrix elements can be determined [14]. 


\section{Appendix B}

\section{Spectral Refractive Indices of Particles}

\section{Constituting Haze and Fog Atmospheric}

Aerosol Models 


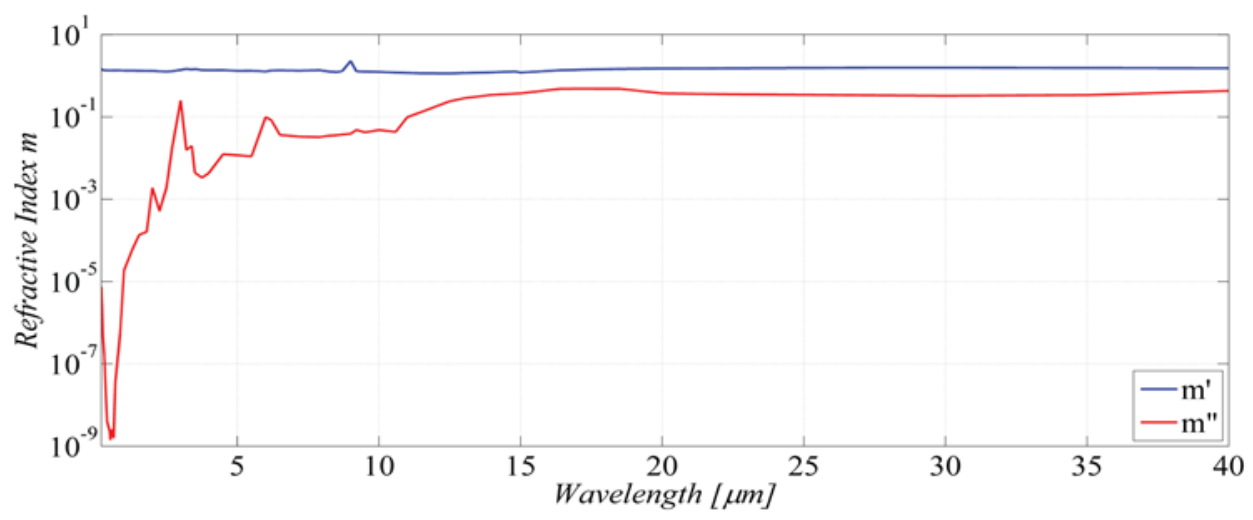

Figure B.1 Maritime haze aerosols, $\mathrm{RH}=90 \%$, real and imaginary parts, $m^{\prime}$ and $m^{\prime \prime}$, of the refractive index, $m=m^{\prime}+i m^{\prime \prime}$.

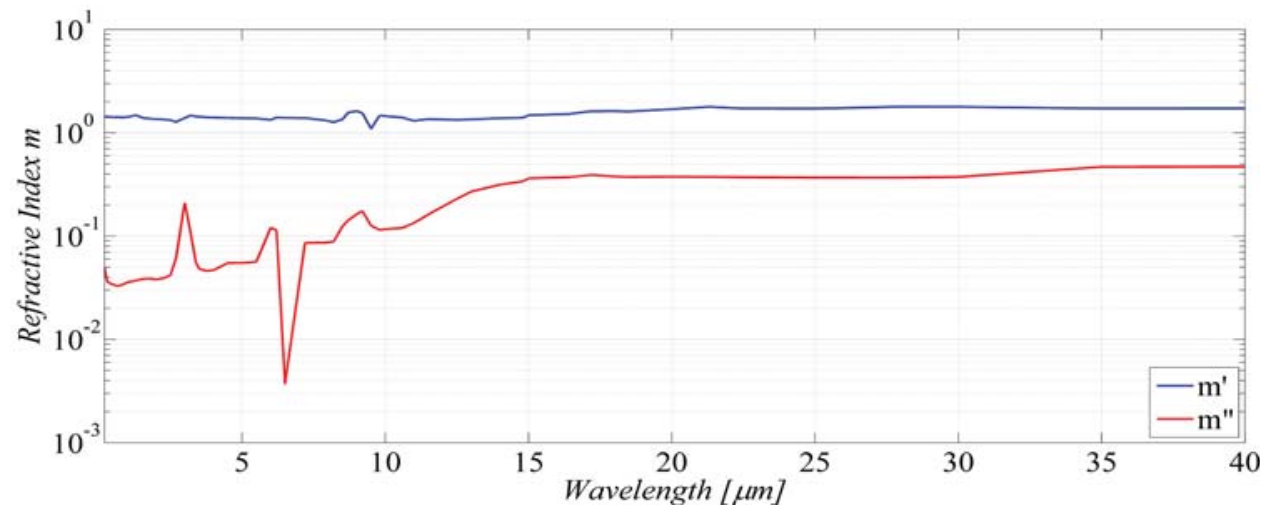

Figure B.2 Urban haze aerosols, $\mathrm{RH}=80 \%$, real and imaginary parts, $m^{\prime}$ and $m^{\prime \prime}$, of the refractive index, $m=m^{\prime}+i m^{\prime \prime}$.

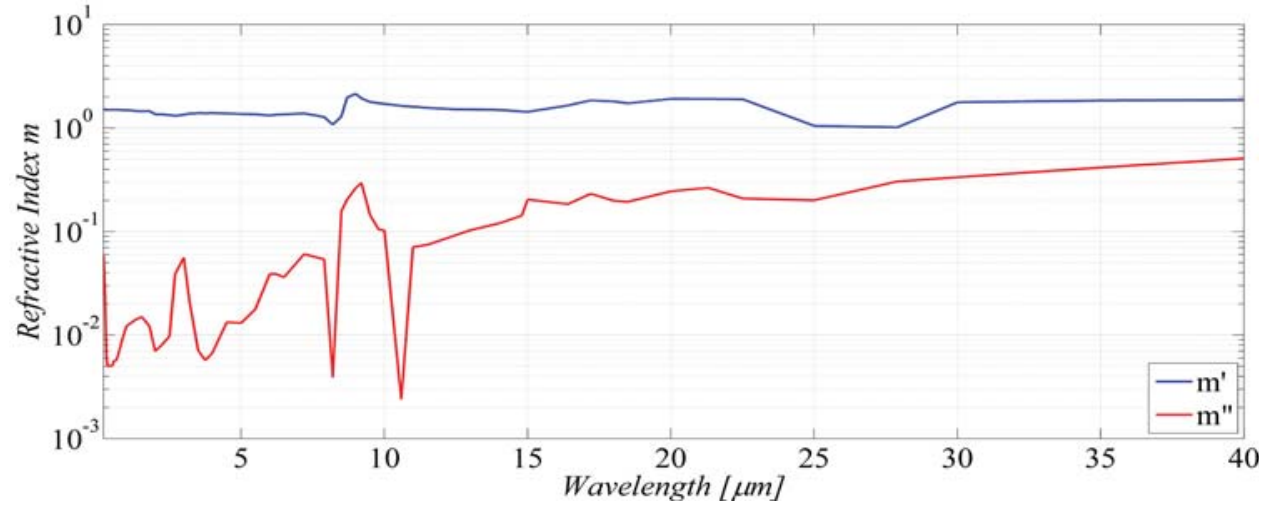

Figure B.3 Rural haze aerosols, $\mathrm{RH}=70 \%$, real and imaginary parts, $m^{\prime}$ and $m^{\prime \prime}$, of the refractive index, $m=m^{\prime}+i m^{\prime \prime}$. 


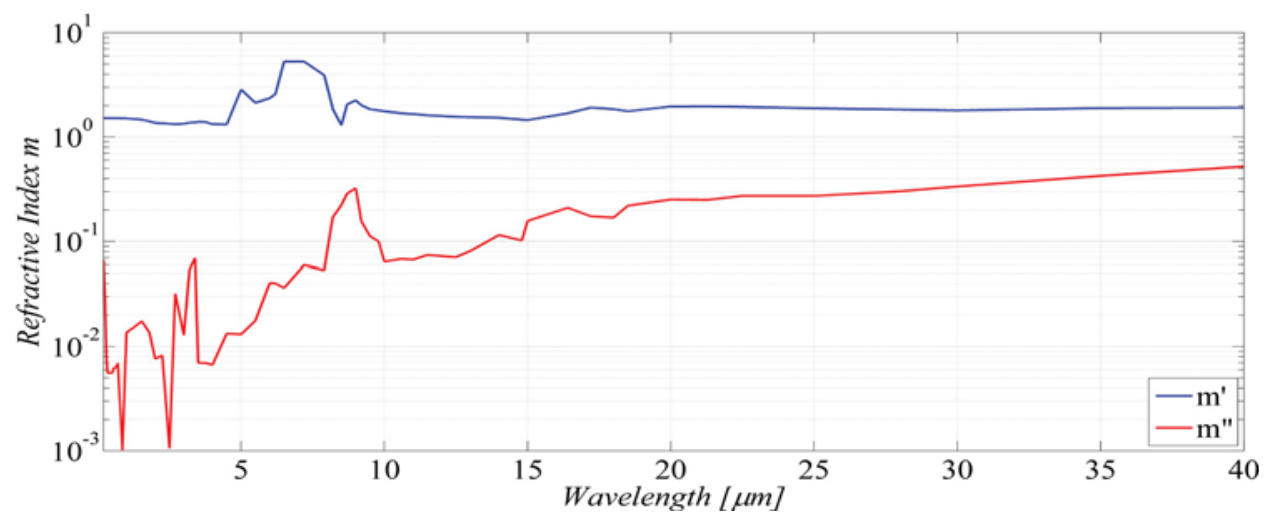

Figure B.4 Tropospheric haze aerosols, $\mathrm{RH}=50 \%$, real and imaginary parts, $m^{\prime}$ and $m^{\prime \prime}$, of the refractive index, $m=m^{\prime}+i m^{\prime \prime}$.

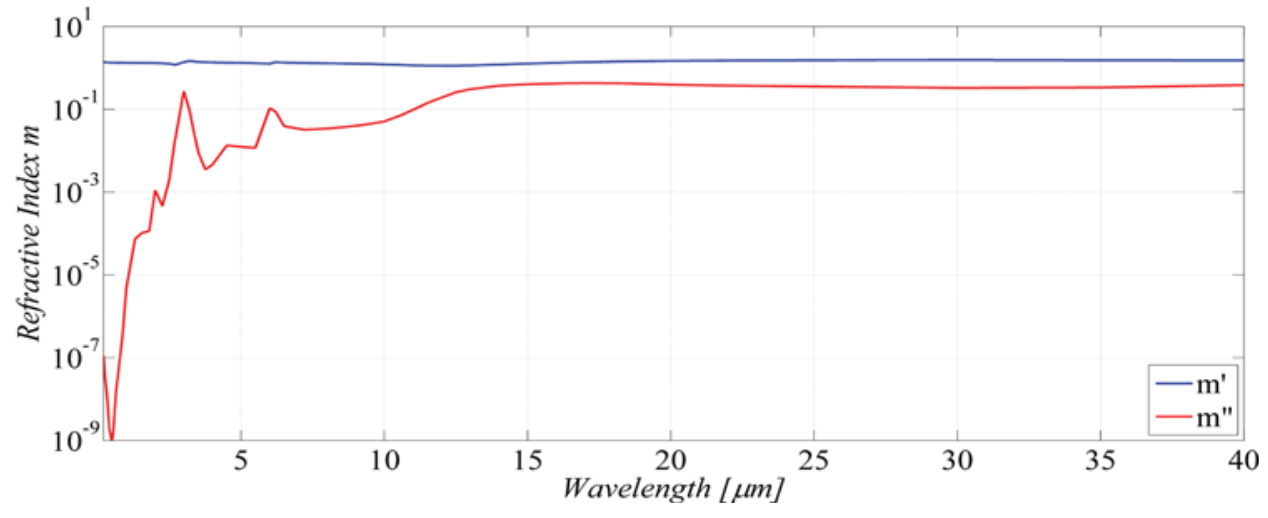

Figure B.5 Fog aerosols, real and imaginary parts, $m^{\prime}$ and $m^{\prime \prime}$, of the refractive index, $m=m^{\prime}+i m^{\prime \prime}$. 


\section{Appendix C}

\section{Letters of Permission}

\section{C.1 OSA letter of permission}

The content in Chapters 2 has been published in the Journal of the Optical Society of America A (JOSA A). OSA has the following policy regarding republication of this material:

As stated in the OSA Copyright Transfer Agreement, OSA grants to the Author(s) (or their employers, in the case of works made for hire) the following rights:

- The right, after publication by OSA, to use all or part of the Work without revision or modification, including the OSA formatted version, in personal compilations or other publications consisting solely of the Author(s') own works, including the Author(s') personal web home page, and to make copies of all or part of the Work for the Author(s') use for lecture or classroom purposes.

- All proprietary rights other than copyright, such as patent rights. 
- If the Work has been prepared by an employee within the scope of his or her employment or as a work made for hire, the right to make copies of the Work for the employer's internal use.

Thus, authors may reproduce figures and text in new publications. The OSA source publication should be referenced.

\section{C.2 SPIE letter of permission}

The content in Chapters 3 and 4 have been submitted to SPIE Optical Engineering. Optical Engineering has the following policy regarding republication of this material:

As stated in the SPIE Transfer of Copyright agreement, authors, or their employers in the case of works made for hire, retain the following rights: All proprietary rights other than copyright, including patent rights.

- The right to make and distribute copies of the Paper for internal purposes.

- The right to use the material for lecture or classroom purposes.

- The right to prepare derivative publications based on the Paper, including books or book chapters, journal papers, and magazine articles, provided that publication of a derivative work occurs subsequent to the official date of publication by SPIE. 
Thus, authors may reproduce figures and text in new publications. The SPIE source publication should be referenced. 